INTERNATIONAL MONETARY FUND

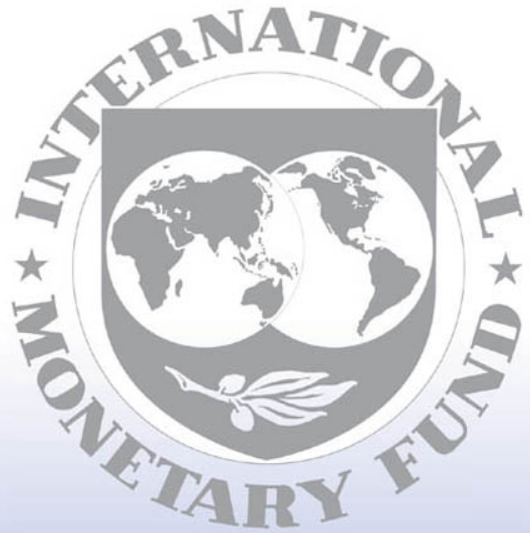

Staff

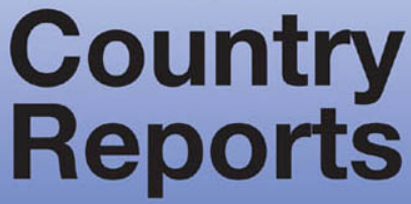




\title{
India: 2009 Article IV Consultation-Staff Report; Staff Statement; Public Information Notice on the Executive Board Discussion; and Statement by the Executive Director for India
}

Under Article IV of the IMF's Articles of Agreement, the IMF holds bilateral discussions with members, usually every year. In the context of the 2009 Article IV consultation with India, the following documents have been released and are included in this package:

- $\quad$ The staff report for the 2009 Article IV consultation, prepared by a staff team of the IMF, following discussions that ended on December 19, 2009, with the officials of India on economic developments and policies. Based on information available at the time of these discussions, the staff report was completed on January 11, 2010. The views expressed in the staff report are those of the staff team and do not necessarily reflect the views of the Executive Board of the IMF.s

- A staff statement of January 25, 2010 updating information on recent developments.

- $\quad$ A Public Information Notice (PIN) summarizing the views of the Executive Board as expressed during its January 25, 2010 discussion of the staff report that concluded the Article IV consultation.

- $\quad$ A statement by the Executive Director for India.

The policy of publication of staff reports and other documents allows for the deletion of market-sensitive information.

\author{
Copies of this report are available to the public from \\ International Monetary Fund • Publication Services \\ $70019^{\text {th }}$ Street, N.W. • Washington, D.C. 20431 \\ Telephone: (202) 623-7430 • Telefax: (202) 623-7201 \\ E-mail: publications@imf.org Internet: http://www.imf.org
}

\section{International Monetary Fund Washington, D.C.}




\section{INTERNATIONAL MONETARY FUND}

\section{INDIA}

\section{Staff Report for the 2009 Article IV Consultation}

Prepared by the Staff Representatives for the 2009 Article IV Consultation with India

Approved by Kalpana Kochhar and Tamim Bayoumi

January 11,2010

Mission. A staff team-K. Kochhar (head), R. Guimarães, L. Papi, M. Saxegaard, P. Topalova (all APD), S. Peiris (MCM), and S. Panth (Senior Resident Representative)—visited New Delhi and Mumbai during December 7-19, 2009.

Past advice. The 2008 Article IV consultation was concluded on February 6, 2009. The Fund supported the authorities' policies in response to the global crisis centered on ensuring the flow of credit to the economy and providing stimulus. Directors, however, underscored the importance of medium-term fiscal consolidation and of reforms aimed at deepening and strengthening the financial sector.

Exchange rate regime. Floating. India is an Article VIII country, but maintains restrictions subject to approval under Article VIII: these restrictions have not been approved by the Executive Board.

Consultation cycle. It is expected that the next Article IV consultation with India will take place on the standard 12-month cycle. 


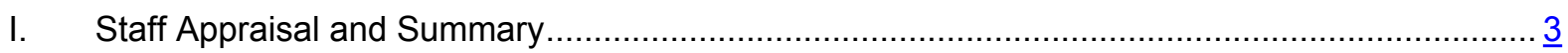

II. Context: On Track to Return to Rapid Growth ............................................................... 4

III. Policies: The Exit Strategy and Key Reforms .............................................................. $\underline{5}$

A. Monetary and Exchange Rate Policy: A Balancing Act ............................................... $\underline{6}$

B. Financial Policies: A More Growth-Oriented Financial System …................................... $\underline{8}$

C. Fiscal Policy: "Fiscal Consolidation is Imperative" ...................................................... 11

\section{Tables}

1. Millennium Development Goals, 1990-2007 ............................................................ 14

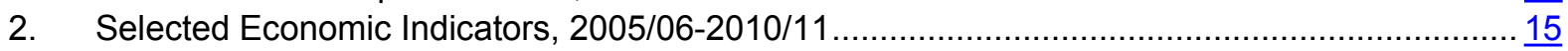

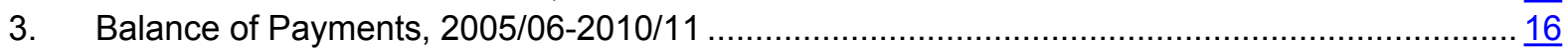

4. Reserve Money and Monetary Survey, 2005/06-2009/10 ........................................... $\frac{17}{18}$

5. Central Government Operations, 2005/06-2009/10 ..................................................... $\frac{18}{19}$

6. General Government Operations, 2005/06-2009/10 .................................................

7. Macroeconomic Framework, 2005/06-2013/14 ......................................................... $\frac{20}{21}$

8. Indicators of External Vulnerability, 2004/05-2009/10 ................................................ 21

9. Indicators of Financial System Soundness, 2004/05-2008/09 …........................................ 22

\section{Appendices}

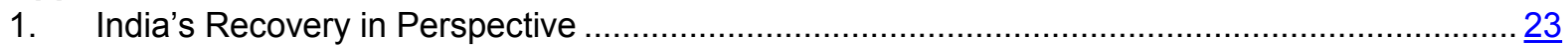

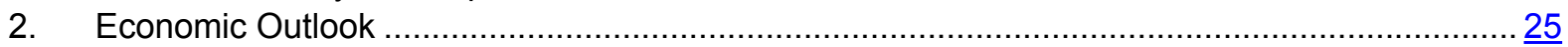

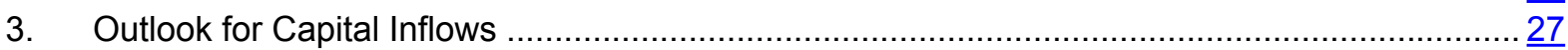

4. Inflation: Developments and Outlook ....................................................................... 28

5. Monetary Conditions, Corporate Funding and Asset Prices ............................................ $\underline{29}$

6. Technical Note on DSGE Model ............................................................................ $\underline{33}$

7. Financial Stability of the Indian Banking System ..................................................... $\frac{34}{37}$

8. Financial Sector Reforms: A focus on Infrastructure Financing ....................................... $\frac{37}{44}$

9. Foreign Participation in Emerging Markets Local Currency Bond Markets............................. $\underline{44}$

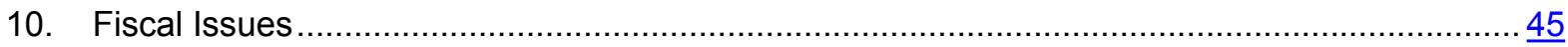

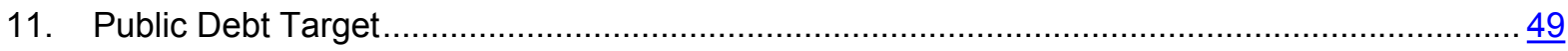




\section{StAff Appraisal AND SUMmary}

1. India's economy is rebounding strongly ahead of most countries in the world, bringing policy trade-offs to a head earlier than in other countries. Growth is approaching pre-crisis levels and leading indicators bode well for continued recovery. Capital inflows are rising and financial markets have regained most of the lost ground. But inflation pressure are intensifying and the fiscal position is one of the weakest among major emerging markets.

2. The exit from the accommodative policies of the crisis should start with:

- a normalization of the monetary stance. Conditions are now ripe for a gradual withdrawal of monetary accommodation to anchor inflation expectations. More extended guidance from the Reserve Bank of India (RBI) on future inflation should also help. Rupee appreciation should be the first response to capital inflows. Other policy options, especially prudential measures, may be appropriate if asset price bubbles are a threat, while tightening capital controls should be a last resort given the need to deepen domestic capital markets.

- a reduction in the $2010 / 11$ budget deficit. With the recovery becoming entrenched and India's high debt, the risk of premature withdrawal of fiscal stimulus is low. Introducing reforms to underpin lasting consolidation will be of paramount importance.

3. The medium-term outlook is bright provided reform efforts are accelerated and sustained. As India was not at the center of the crisis and its growth is mainly reliant on domestic drivers, the global crisis is not expected to have dented India's medium-term growth potential: real GDP growth is projected to return to 8 percent in 2010/11 with near-term risks broadly balanced. Nevertheless, to sustain rapid growth over the longer term, the authorities have rightly emphasized addressing structural bottlenecks—notably infrastructure. The government is also addressing other structural issues, such as land acquisition, and education and judicial reforms.

4. The main economic reform objectives should be: (i) enabling the financial system to intermediate domestic savings effectively; and (ii) reducing fiscal dominance.

- a more growth-oriented financial system. The financial system has weathered the global crisis well and appears sound, despite deteriorating asset quality, which would benefit from strengthening mechanisms to deal with distressed assets. Nevertheless, as investment recovers and infrastructure takes off, the limitations of the domestic financial system may come to the fore. Deepening the domestic corporate bond market is key, as well as the availability of instruments that help manage risks, and greater participation of domestic institutional investors in the funding of infrastructure.

- $\quad$ reducing the public sector's claim on resources. A framework anchored in a debt target, in the order of 60-65 percent of GDP by 2015 , accompanied by expenditure ceilings would stand a better chance of achieving fiscal discipline while allowing countercyclical policy. A durable consolidation hinges on a reform to remove regressive subsidies and the introduction of the planned GST and the new Direct Tax Code. Ensuring high value for money of social programs will be important to achieve significant poverty reduction. Privatization would also help consolidation. 


\section{Context: on TRACK to RETURn to RAPID GROWTH}

5. India's economy is among the first in the world to recover after the global crisis (see Appendix 1). Prompt fiscal and monetary easing, combined with the fiscal stimulus already in the pipeline and the return of risk appetite in financial markets have brought growth close to pre-crisis levels. Leading indicators suggest the output gap will continue to close. Capital inflows are on the rise, and financial markets have regained most of the lost ground.
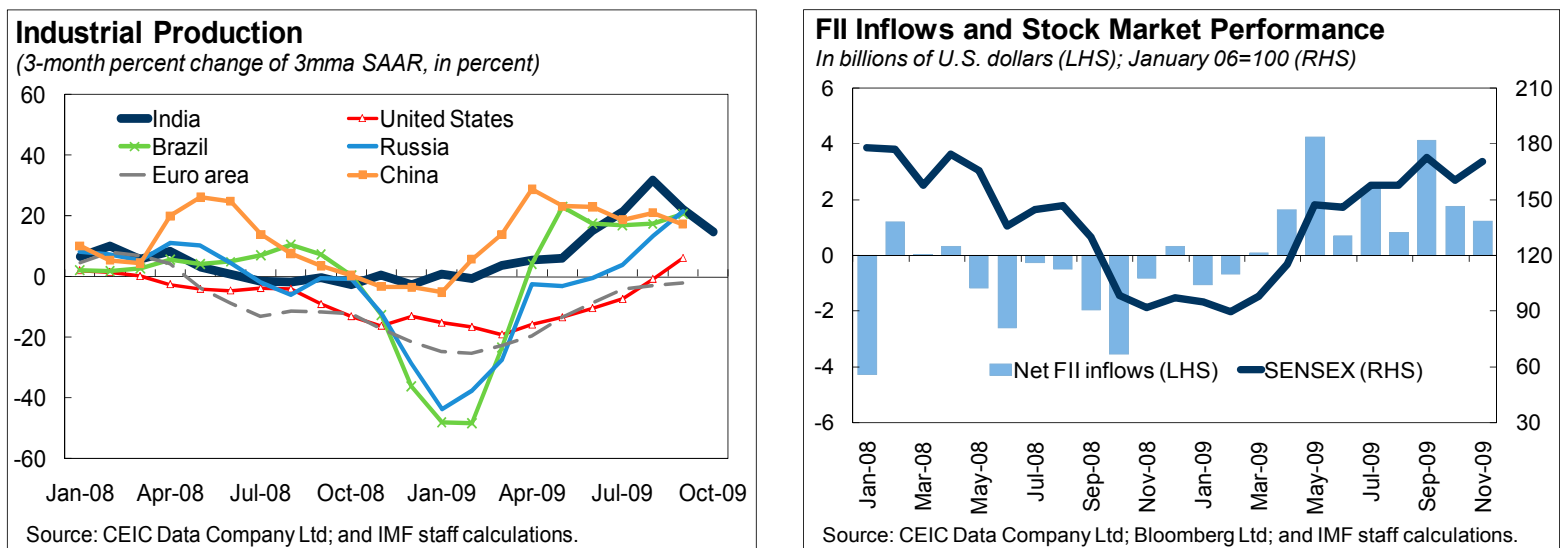

6. Growth is projected to rise from $63 / 4$ percent in $2009 / 10$ to 8 percent the following year (see Appendix 2). Agriculture is likely to contract by about 1 percent in 2009/10 due to the drought, but nonagricultural GDP growth is expected to gather momentum. Private consumption would benefit from better employment prospects in industry and services and less uncertainty, and investment would be boosted by robust corporate profits, rising business confidence, and favorable financing conditions (see Appendix 3). With India's long-term prospects remaining strong and private sector balance sheets sound, we expect growth to be back at potential in 2010/11 even if advanced economies grow below trend. The current account deficit will stay relatively stable over the medium term, with export and import growth gathering pace.

India: Summary of Medium-Term Macroeconomic Framework, 2007/08-2014/15

\begin{tabular}{|c|c|c|c|c|c|c|c|c|}
\hline & \multirow[b]{2}{*}{$2007 / 08$} & \multirow[b]{2}{*}{$2008 / 09$} & \multicolumn{6}{|c|}{ Proj. } \\
\hline & & & $2009 / 10$ & $2010 / 11$ & $2011 / 12$ & $2012 / 13$ & $2013 / 14$ & $2014 / 15$ \\
\hline \multicolumn{9}{|l|}{ Real sector (percent change) } \\
\hline Real GDP growth & 9.0 & 6.7 & 6.7 & 8.0 & 7.7 & 7.8 & 8.1 & 8.1 \\
\hline Real GDP growth, agriculture & 4.9 & 1.6 & -0.6 & 4.7 & 2.7 & 2.7 & 2.7 & 2.7 \\
\hline Real GDP growth, nonagriculture & 9.9 & 7.8 & 8.2 & 8.6 & 8.6 & 8.7 & 8.9 & 8.9 \\
\hline Inflation (period average) & 4.6 & 8.3 & 3.1 & 6.4 & 4.6 & 4.0 & 4.0 & 4.0 \\
\hline Inflation (end of period) & 7.7 & 0.8 & 8.1 & 5.4 & 4.0 & 4.0 & 4.0 & 4.0 \\
\hline \multicolumn{9}{|c|}{ Saving and investment (in percent of GDP) } \\
\hline Gross domestic investment & 39.1 & 40.0 & 39.2 & 40.3 & 41.1 & 41.9 & 42.9 & 43.9 \\
\hline Gross national saving & 37.6 & 37.4 & 37.4 & 38.2 & 39.3 & 40.2 & 41.1 & 42.3 \\
\hline Current account (in percent of GDP) & -1.3 & -2.5 & -1.9 & -2.1 & -1.8 & -1.8 & -1.8 & -1.8 \\
\hline
\end{tabular}

Source: IMF staff estimates.

7. Near-term risks are broadly balanced. On the upside, an acceleration of reforms and capital inflows could spur investment. On the downside, the main risks are elevated inflation and financing constraints arising, inter alia, from the fiscal deficit, which could stall the recovery. Tail risks include asset price bubbles and a sudden stop in capital inflows caused by turmoil in global financial markets. 
8. India's medium-term growth prospects remain bright. India was not at the center of the global crisis and growth is well balanced and mainly reliant on domestic drivers. Risks to this favorable outlook stem primarily from difficulties in implementing productivity-enhancing reforms and continued supply bottlenecks.

\section{Authorities' Views}

9. The authorities see India's recovery well underway and their growth projections are higher than those of staff. They attribute this to a combination of decisive policy measures and the fact that India has only been indirectly affected by the crisis. The authorities project 2009/10 growth at 7-7.75 percent. In a recent speech, the PM noting that "there are clear signs of an upturn in the economy," anticipated growth at over 7 percent for $2010 / 11 .{ }^{1}$

10. The authorities are concerned about rising inflation in the food sector, although the RBI expects year-end inflation to be lower than staff's 8 percent projection. The RBI estimates that WPI inflation will be 6.5 percent with an upward bias by March 2010, but they note that it is mainly a supply-side phenomenon linked to food prices. They are aware of the risks of the spillover of persistently high food prices, which have been rising for over a year, into nonfood prices via their effect on expectations-demand side factors are also at play.

11. Weakness in advanced economies' activity, the rise in commodity prices, and the likelihood of large capital inflows are viewed as the main risks to the near-term outlook. The recovery is still driven by government spending (with signs of pickup in private final consumption demand in the second quarter) and the recovery in IT and BPO sectors, which have large spillover effects on the rest of the economy, hinges on growth in advanced economies. So far the levels of government borrowing have been managed without any adverse impact in terms of crowding out the resources for the private sector. Going forward, the proposed resumption of the fiscal consolidation process presently envisaged by the Medium Term Fiscal Policy Statement would minimize the risks of crowding out in the event of a pickup in credit growth.

\section{Policies: The Exit Strategy and Key Reforms}

12. Emerging inflation pressures and a weak fiscal position are bringing policy trade-offs to a head earlier than in other countries. Headline (WPI) inflation, running at 10 percent sequentially, is fuelling debate on when the Reserve Bank of India (RBI) should tighten monetary policy. The double-digit fiscal deficit and high public debt call for fiscal consolidation, but there is a risk that private demand may not broaden enough to carry the growth momentum. Capital inflows help fund investment, but could complicate monetary management.

\footnotetext{
${ }^{1}$ PM's address at the inauguration of India Economic Summit, November 8, 2009.
} 

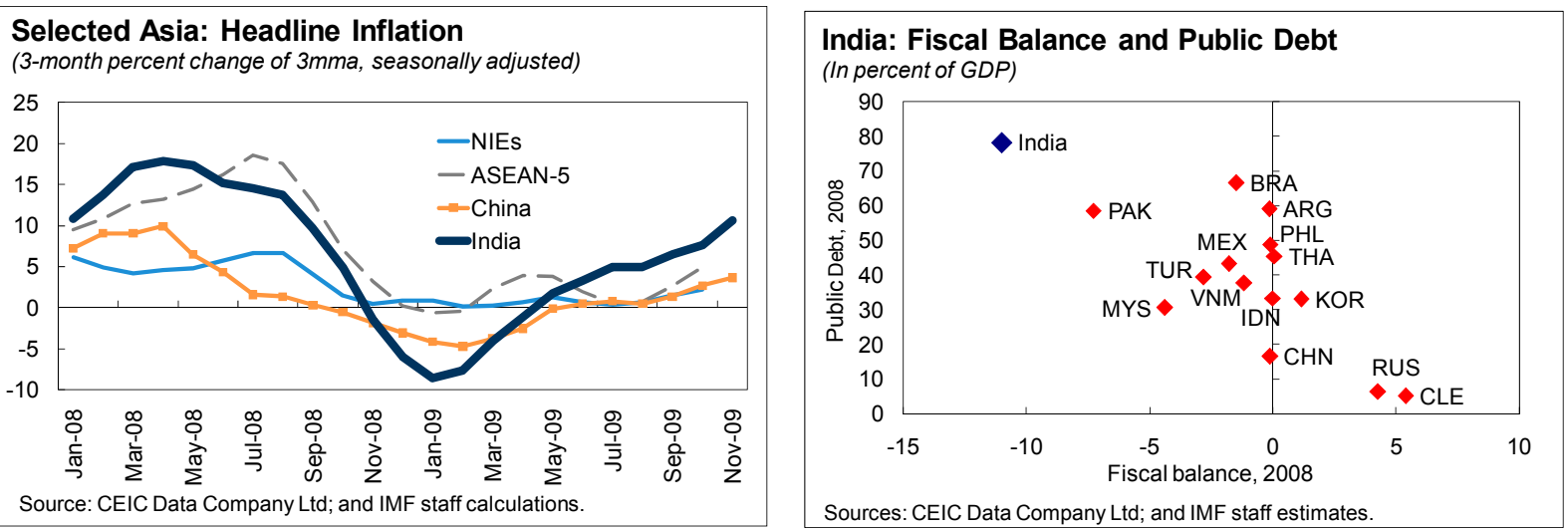

13. The 2009 Article IV consultation focused on these immediate challenges and the transition to the longer-term economic reform agenda. India, with its twin-engined growth (from domestic demand and exports), is well-positioned to grow faster than comparators for whom external demand weakness will be a drag and the process of rebalancing growth slow. To capitalize on this opportunity, India needs to address structural weaknesses. The government aims at rapid and inclusive growth and has identified infrastructure bottlenecks as the main supply constraint. As the Prime Minister stated, the country is better placed than at any time in the recent past to push reforms. We see two priorities: (i) financial reform to facilitate infrastructure and investment; and (ii) fiscal reforms to free resources for public and private investment and increase the efficiency of public spending. Besides these, the government is also working on addressing other structural issues, such as land acquisition, and education and judicial reforms.

\section{A. Monetary and Exchange Rate Policy: A Balancing Act}

14. The RBI has begun the first phase of exit. It eliminated some unconventional financing facilities introduced but little used during the crisis, re-introduced caps on the cost of external borrowing, and signaled its intention to return to a neutral monetary stance. It also reversed the cut in the statutory liquidity ratio, and raised provisioning requirements on commercial real estate.

15. Conditions are ripe for a normalization of the monetary stance.

- Inflation (see Appendix 4). CPI inflation has been in the low double digits for over a year and WPI has risen fast. Though food accounts for a significant part of the increase in inflation, the output gap is estimated to be small and expected to close by end-2010/11, core inflation has risen rapidly, and so have survey-based inflation expectations. March 2010 WPI inflation is projected at 8 percent $\mathrm{y} / \mathrm{y}$, with commodity prices being the main risk.

- $\quad$ Activity. PMls for manufacturing and services have been in expansionary territory since April-May 2009, with the employment component also above neutral.

Nonagricultural output growth is projected at around 9 percent $y / y$ for the rest of the

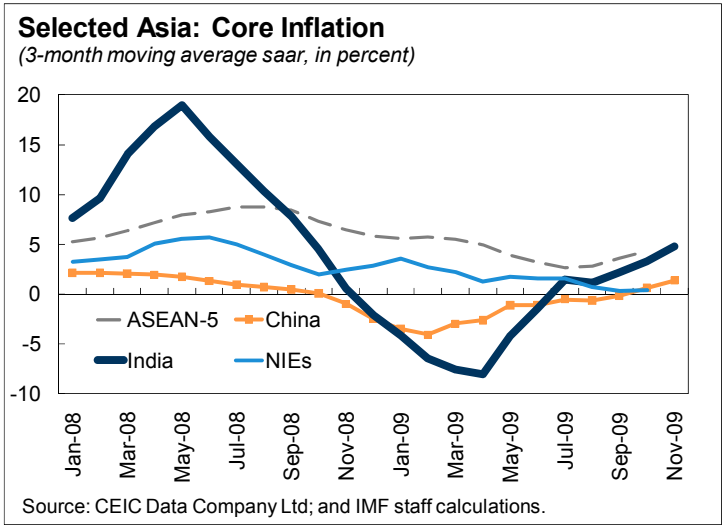
current fiscal year. Corporate issuance in capital markets has increased substantially, albeit 
from low levels, as asset prices have rallied. Credit growth is weak, but past episodes of slowdowns in several countries suggest that credit growth lags the recovery (see Appendix 5).

16. The withdrawal of monetary stimulus should be gradual, but should start soon. The uncertainty about the durability of the recovery calls for reducing the degree of monetary accommodation in small steps. But, monetary policy transmission lags and the fact that the policy rate is substantially below the neutral rate (estimated using a simple Taylor rule) argue for timely action. Moving early would also mitigate the risk of inflation expectations becoming unmoored, and thus would require a smaller overall adjustment. Initial steps could be to sequester most of the excess liquidity (which averaged Rs1.1 trillion in April-December 2009) through increases in the cash reserve ratio-this could strengthen monetary transmission. The RBI could also provide its one-year ahead inflation projection in every quarterly review to guide inflation expectations.

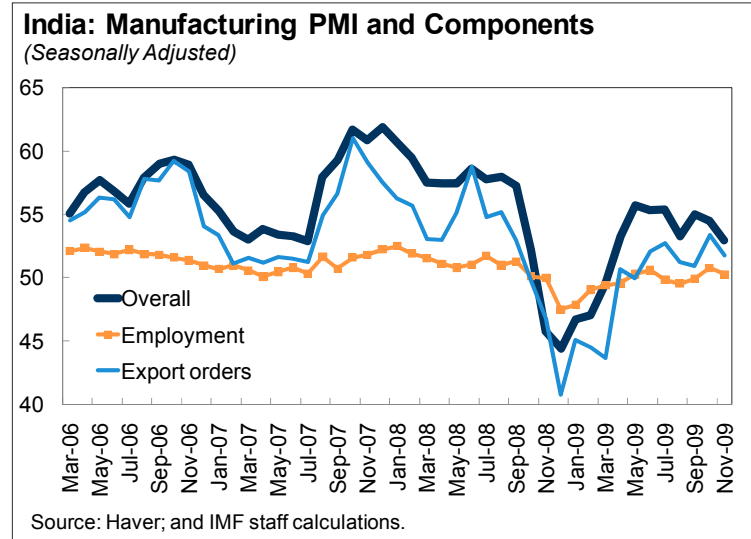

17. Rupee appreciation would contain inflation, could allow the RBI to keep interest rates low for longer, and would help manage capital inflows. Given low interest rates in advanced economies and India's high relative growth, capital inflows are likely to be substantial. With the rupee still below its 2008 peak, $^{2}$ there would be room for appreciation without concerns about eroding competitiveness. Sterilized intervention is costly and may encourage further inflows by maintaining expectations of appreciation and by increasing risk-adjusted returns. Prudential measures, which the RBI employs, could be used to dampen incipient asset price bubbles. And finally, given the need to develop India's financial markets and finance investment, we would advise using capital controls only as a last resort.

18. Staff studies suggest that the weight on exchange rate changes in the setting of monetary policy could be lowered (see Appendix 6). A Dynamic Stochastic General Equilibrium (DSGE) model estimated for India indicates that macroeconomic volatility would be reduced by responding more to inflation relative to the exchange rate.

\footnotetext{
${ }^{2}$ From a longer-term perspective, the Consultative Group on Exchange Rate (CGER) estimates that the rupee is broadly in line with its value based on fundamentals ( 0 to 10 percent below equilibrium). Exports shares of foods and services have steadily increased since the early 1990 s and external debt remains low.
} 


\section{Authorities' Views}

19. The RBI has already started exiting from crisis measures and is shifting towards a more neutral monetary stance. Monetary policy is unlikely to be able to affect food prices. Hence, monetary settings should continue to support the economic recovery and "it may be appropriate to sequence the exit in a calibrated way so that while the recovery process is not hampered, inflation expectations remain anchored." ${ }^{3}$

20. The authorities underscore that the exchange rate is largely market determined and they intervene only to reduce excessive volatility of the exchange rate rather than managing the level of the exchange rate. They also pointed out that appreciation would not help too much in controlling food prices because of limited tradability of key items.

21. The RBI will continue to adopt a range of measures to manage capital inflows as needed. While inflows are currently in line with the economy's absorptive capacity and are not posing problems for monetary management, the authorities remain cognizant of their impact on liquidity and the exchange rate. Given their experience during the surge in capital inflows in 2007, the authorities are confident they would effectively manage the expected inflows. The policy options on debt flows remain a tool to be used. Asset prices are not considered frothy, but their role in policy formulation is likely to be more prominent, with macro-prudential measures seen as the right instrument to address incipient asset price bubbles.

\section{B. Financial Policies: A More Growth-Oriented Financial System}

22. Based on the authorities' self assessment, ${ }^{4}$ India's financial system appears sound, despite some recent deterioration in asset quality (see Appendix 7). Financial soundness indicators compare well internationally, except for provision coverage (the RBI recently raised the requirement), although the comparison of nonperforming assets (NPAs) is complicated by forbearance. ${ }^{5}$ Staff's analysis indicates that the capital of the top 30 banks would remain above the regulatory minimum in a scenario in which NPAs double to 5 percent, and only some smaller banks would need more capital in a more extreme scenario in which NPAs triple. Credit remains the key risk, but the authorities have started to strengthen public banks' capital and financial regulation following the Financial Stability Board (FSB) guidelines (liquidity risk monitoring and disclosure of offbalance sheet exposures). Given the global experience, multi-factor stress tests and an independent evaluation of compliance with international standards would be advisable. The RBI's new Financial Stability Unit should also help achieve a better integration of micro- and macro-prudential surveillance.

\footnotetext{
${ }^{3}$ RBI's Second Quarter Review of Monetary Policy, October 27, 2009.

${ }^{4}$ The Committee on the Financial Sector Assessment (2009), "India's Financial Sector: An Assessment”, carried out a comprehensive analysis of financial stability and standards and codes, which was subject to extensive peer review by independent advisors.

${ }^{5}$ About 3 percent of loans were rescheduled since October 2008.
} 
23. As infrastructure investment takes off, a priority is to ensure that the domestic financial system stays ahead of the needs (see Appendix 8). While India's savings are relatively high, about a third is in physical assets: hence deepening the financial system and its ability to intermediate more efficiently is critical. Also, the public sector is expected to continue to absorb substantial resources at the same time as private credit demand picks up. Increases in banks' infrastructure exposure is constrained by maturity mismatches and prudential exposure limits. Financial instruments and mechanisms typically used in infrastructure funding—such as long-term bonds, takeout financing, securitization, and credit enhancements-are limited.

24. Steps taken by the authorities to address these constraints are welcome. The planned introduction of a takeout financing scheme by the India Infrastructure Finance Corporation Limited (IIFCL) and the reduction in capital requirements for this instrument, the measures recently taken to give greater flexibility to certain pension funds in their investments, as well the recent RBI guidelines on CDS and repos with corporate bonds will help promote long-term debt financing. Similarly, the introduction of interest rate and currency futures provides additional instruments to manage market risk.

25. Domestic institutional investors hold the key to the development of the corporate bond market. India's corporate bond market is small compared to other EMs due to a limited investor base and investment restrictions, the absence of a benchmark yield curve, and inadequate credit enhancement and hedging tools. International experience suggests that institutional investors have been instrumental to the growth of the corporate bond market, and, in turn, to the provision of development finance. Pension funds' assets were critical in Chile and Mexico, while in Malaysia corporate bond issuance was helped by easing of investment regulations on insurance companies. In India too, institutional investors are growing rapidly, and passage of the insurance and Pension Fund Regulatory and Development Authority bills would encourage further growth. Nevertheless, their role as captive sources for government financing would need to be addressed and investment restrictions eased. In addition, the supply of high grade bonds would have to be boosted, including via suitable credit enhancements.

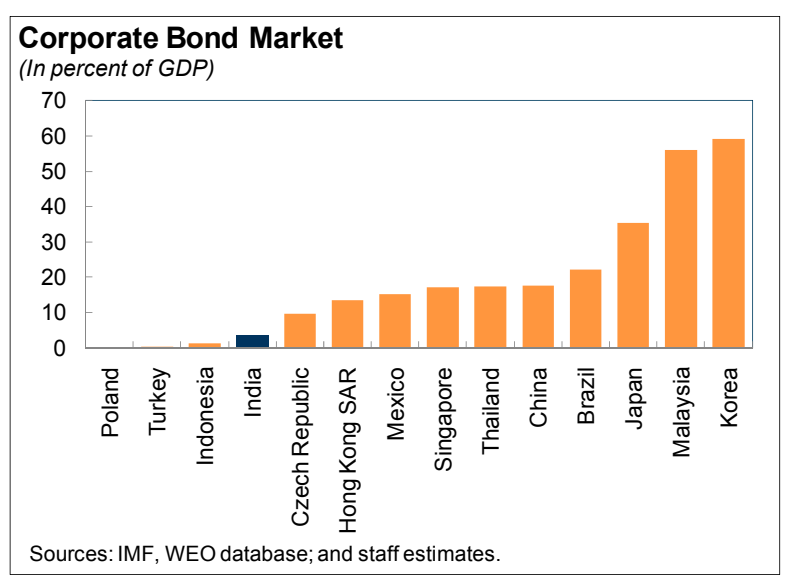

26. Foreign participation is vital to enhance liquidity, the sine qua non of a vibrant bond market. Domestic institutional investors, as in most countries, will tend to be buy-and-hold investors. Hence, opening the debt market to foreign investors could enhance liquidity-a staff study shows that greater foreign participation lowers yields, but there is no clear evidence that volatility increases (see Appendix 9). Merging FII limits for the corporate and government bonds and liberalizing the allocation mechanisms could help in this regard. Even if initially investors are likely to prefer government bonds, foreign participation should develop the sovereign yield curve facilitating the pricing of corporate debt. 
Further, consideration could also be given to liberalizing external borrowing for refinancing of infrastructure loans, as this would be more likely to attract foreign capital in infrastructure and free domestic resources for other uses.

27. Public institutions have an important role to play in establishing the overall framework and in filling market gaps in the provision of infrastructure financing.

- Institutional framework to drive the implementation of infrastructure investment. The secretariat to the cabinet committee on infrastructure chaired by the Prime Minister could be assisted by a multi-disciplinary team, empowered to address infrastructure financing challenges. This team would identify bottlenecks and coordinate the relevant policy makers to create a modern framework for infrastructure financing, being mindful of financial stability.

- $\quad$ Filling market gaps. Given the large infrastructure needs, the role of public institutions should be mainly catalytic of private financing, in order to leverage their resources. In this regard, the fact that the IIFCL is planning to provide takeout finance and credit enhancements, whose supply by other players is likely to be limited, is an encouraging development. The same could be done with other specialized public institutions. The risks incurred by the public sector would have to be carefully monitored and managed.

28. Improving mechanisms to deal with distressed assets and insolvency proceedings remain important. The market for distressed assets could be boosted by further improving the insolvency framework for non-bank finance companies and easing restrictions on investment in asset reconstruction companies. Passage of the new Companies Bill would strengthen the insolvency framework.

\section{Authorities' Views}

29. As concluded by the Report of the Committee on Financial Sector Assessment (CFSA), the authorities consider that India's financial system is sound and resilient. The authorities deem that there are no significant vulnerabilities in the system and that the situation has further improved since the CFSA Report. Though NPAs may increase as a result of the slowdown, the rise is unlikely to pose systemic risks given the strength of banks' balance sheets. To further strengthen financial stability, the RBI will issue guidelines to banks on enhanced stress testing by end-January 2010 and enhance management of liquidity and interest rate risks.

30. India continues to strengthen prudential regulation and supervision of banks. In the October policy review, the RBI raised the provisioning requirement for advances to the commercial real estate sector given the high credit growth and the extent of restructured loans in this sector. The $\mathrm{RBI}$ also advised banks to raise their total provisioning ratio, including floating provisions, to a minimum of 70 per cent by September 2010 with a view to improving the provisioning cover and enhancing the soundness of individual banks. To ensure that the originators do not compromise on due diligence of assets generated for the purpose of securitization, it has been proposed to stipulate a minimum lock-in period of one year and a minimum retention of 10 percent for bank loans that are securitized.

31. A number of steps have been taken to introduce new financial products and improve the efficiency of the banking system. Stock exchanges are now permitted to offer currency futures contracts in currency pairs of Rupee-Euro, Rupee-Pound Sterling and Rupee-Japanese Yen, in addition to the existing Rupee-US dollar contracts. In addition, the authorities have re-launched interest rate futures (IRFs) on 10-year government bonds with improved features in August 2009. The $\mathrm{RBI}$ is also planning to introduce plain vanilla over-the-counter (OTC) single-name CDS for corporate bonds subject to appropriate safeguards and repos of corporate bonds. An RBI Working Group has 
been established to examine the issues involved in the introduction of priority sector lending certificates, while a committee has recommended a new formula to replace the system of benchmark prime lending rate and use the cost of one-year retail deposits as the benchmark for calculating the proposed benchmark lending rate. The authorities are also working on several measures that would enhance financial inclusion.

32. The authorities maintain that the country's infrastructure financing needs are currently being met, but they concur that there is need for improvement. At present, there is no shortage of infrastructure financing and domestic banks-together with the specialized financial institutions set up by the government-are able to bring projects to financial closure without undue delays. Additional instruments are being introduced on an as needed basis: for example, IIFCL is working on providing takeout financing and credit enhancements. The authorities agree that developing a corporate bond market continues to be key to creating sources of long-term debt financing, but do not see merging the FII limits between the corporate and government bond markets as helpful. The Government of India has set up a High Level Standing Committee on Infrastructure Finance to oversee the recommendations on infrastructure financing and to act as the coordinating institutional mechanism to review the bottlenecks and the resource availability in the infrastructure sector. Finally, a Cabinet Committee on Infrastructure has also been constituted underscoring that the government is according top priority to infrastructure investment and a number of measures (including those related to financing issues) are being taken to expedite projects.

\section{Fiscal Policy: "Fiscal Consolidation Is Imperative"}

33. The 2009/10 budget extended the expansionary stance to support the recovery. Following an estimated deficit of 11 percent of GDP in 2008/09, the general government is likely to end the current fiscal year with a broadly unchanged balance (see Appendix 10). At the central level, savings from the lower subsidy bill (about 2 percent of GDP) have been reoriented towards social programs and infrastructure. Tax revenue is expected to remain subdued reflecting indirect tax cuts introduced during the crisis. States' deficits are also likely to widen.

34. The Finance Minister's announced intention to lower the central government's deficit in the next two budgets is welcome, but is likely to require discretionary measures. While the exact path of fiscal consolidation will be informed by the recommendations of the $13^{\text {th }}$ Finance Commission, the authorities' current goal is to reduce the center's deficit from a projected 7 percent of GDP in 2009/10 to 5.5 percent of GDP in 2010/11 and further to 4 percent of GDP in 2011/12. This will be helped by the falling away of one-off expenditure items and revenue buoyancy, but additional discretionary measures will likely be needed. States will also need to curb current spending.

35. A concrete strategy for reducing debt and the public sector's claim on the economy's resources-consistent with the $\mathbf{G} 20$ principles ${ }^{7}$ - will go a long way to boost credibility. ${ }^{8}$ In India's case, the risks of a premature withdrawal of fiscal stimulus need to be balanced against the possibility that fiscal consolidation—based on durable reforms - could actually be expansionary. A medium-term fiscal framework anchored in an explicit debt target complemented by expenditure rules

\footnotetext{
${ }^{6}$ Minister of Finance, November 10, 2009.

${ }^{7}$ Group of Twenty, Global Economic Prospects and Principles for Policy Exit, Meeting of G-20 Finance Ministers and Central Bank Governors, November 6-7, 2009, St. Andrews, United Kingdom.

${ }^{8}$ While a gradual decline in India's public debt-to-GDP ratio is projected under various shocks, a no-policy change scenario would result in an unsustainable debt path (see Informational Annex No.1).
} 
stands a better chance of achieving fiscal discipline. ${ }^{9}$ Expenditure ceilings, derived from an over-thecycle deficit consistent with the debt target, would introduce a countercyclical element to fiscal policy. Although determining an appropriate debt target is challenging, cross-country analysis using a range of approaches suggests that a target of 60-65 percent of GDP by 2015/16 from 78 percent in 2008/09 might be suitable for India (see Appendix 11). This ceiling would be above the average debt of EMs, but would provide room for countercyclical fiscal policy and for contingent liabilities. It would also send a strong signal by making a clear break with the past.

36. A durable reduction in the fiscal deficit hinges on subsidy reform, although the planned revenue reforms will also contribute. The draft Direct Tax Code would broaden the tax base, while lowering rates. The Goods and Services Tax will streamline indirect taxes. Both reforms should bolster revenue via efficiency gains and improved compliance. The missing element is a reform of the subsidy system, which should phase-out regressive subsidies and introduce a marketbased pricing mechanism for petroleum products in line with G-20 principles. ${ }^{7}$ Over time, the subsidies on products consumed predominantly by the poor could be replaced by targeted support which will be facilitated by the Unique Identification Number. The government's focus on raising the quality of public spending (through consolidation, monitoring, and evaluation of centrally sponsored schemes) should improve service delivery, critical for inclusive growth and poverty alleviation. A faster pace of privatization would also support fiscal consolidation by contributing a one-off increase in resources, but cannot take the place of adjustment.

\section{Authorities' Views}

37. The authorities highlight that fiscal policy has played a major role in cushioning the impact of the crisis and supporting the economic recovery. The fiscal consolidation in the five years leading to the crisis had created the space for the continued expansionary fiscal stance in the $2009 / 10$ budget. The central government 2009/10 fiscal deficit is expected to remain within the 6.8 percent of GDP target. Stronger divestment receipts and direct tax revenue could make up for the shortfall, if any, in $3 G$ auction proceeds and indirect tax collections, while higher than budgeted spending on pensions and food and fertilizer subsidies can be accommodated through savings on other accounts. The record market borrowing program has proceeded in a non-disruptive manner, with limited impact on bond yields. Most of the required resources were raised in the first half of the fiscal year to ensure adequate space for the private sector when private demand rebounds.

38. However, the authorities uphold that fiscal consolidation is imperative. Given the risks associated with a premature exit, a decision regarding the withdrawal of the stimulus measures will be taken without putting at risk the revival process, incorporating the $13^{\text {th }}$ Finance Commission's recommendations on India's medium-term path of fiscal consolidation and discussing expenditure management measures and initiatives to boost revenue.

39. Current plans envisage a reduction in the central government deficit to 5.5 percent of GDP in 2010/11 and 4 percent in 2011/12, as noted in the Medium-Term Fiscal Policy Statement. Relative to $2009 / 10$, spending will be contained as the arrears related to the Pay commission award will be paid off, while the frontloading of the $11^{\text {th }}$ Five Year Plan Programs will result in lower expenditure growth. The pick-up in economic activity will also buoy tax collections. In addition, the government's new divestment policy should raise substantial amount of resources that would help limit the public borrowing requirement.

\footnotetext{
${ }^{9}$ Simone A. and P. Topalova, 2009, "India's experience with fiscal rules: an evaluation and the way forward" IMF Working Paper 09/175.
} 
40. The authorities concur that medium-term fiscal policy should be enshrined in a fiscal rules framework to ensure a lasting fiscal consolidation. They reiterate the important role that the FRBMA has played in reducing the fiscal deficit prior to the crisis. The exact contour of the mediumterm fiscal framework will be traced in the $13^{\text {th }}$ Finance Commission report (expected to be publicly released in February 2010). However, the authorities are receptive to staff's recommendation to anchor the fiscal framework to a medium-term debt target of about 65 percent of GDP and bring all spending above the line.

41. The authorities view the introduction of the GST and Draft Direct Tax Code as having an enormous potential to raise economic efficiency and thus contribute to fiscal consolidation in the medium term. Despite considerable progress, the GST will unlikely be introduced by April 2010 , as its exact design-including the number and level of rates, and tax base-is still being worked out between the center and the states. The draft Direct Tax Code is currently being revised to incorporate comments from stakeholders. Both reforms will broaden the tax base, while lowering tax rates, which will improve compliance, reduce administrative costs, and enhance efficiency.

42. The authorities agree that a durable fiscal consolidation would focus not only on revenue improvement but also on expenditure management. The government remains committed to reducing the subsidy bill. Reform options are being explored, as evidenced by the setting up of an expert group to examine fuel pricing and the proposed adoption of a nutrient-based fertilizer subsidy regime with the goal of moving to a system of direct transfers to the farmers. With regards to plan spending, there is a renewed focus on expenditure management in order to further improve the quality of spending. 
Table 1. India: Millennium Development Goals, 1990-2007 1/

\begin{tabular}{|c|c|c|c|c|c|c|c|}
\hline & 1990 & 1995 & 1998 & 2001 & 2004 & 2006 & 2007 \\
\hline \multicolumn{8}{|l|}{ Eradicate extreme poverty and hunger $2 /$} \\
\hline $\begin{array}{l}\text { Income share held by lowest } 20 \% \\
\text { Malnutrition prevalence, weight for age ( } \% \text { of children under } 5 \text { ) }\end{array}$ & $\ldots$ & $\ldots$ & 44.4 & $\ldots$ & 43.5 & $\cdots$ & $\ldots$ \\
\hline $\begin{array}{l}\text { Malnutrition prevalence, weight for age (\% of children under } 5) \\
\text { Poverty headcount ratio at national poverty line (\% of population) }\end{array}$ & $\ldots$ & 36.0 & 44.4 & 28.6 & 27.5 & $\cdots$ & $\cdots$ \\
\hline Prevalence of undernourishment (\% of population) & $\ldots$ & 24.0 & 21.0 & $\ldots$ & $\ldots$ & $\ldots$ & $\ldots$ \\
\hline \multicolumn{8}{|l|}{ Achieve universal primary education $3 /$} \\
\hline Literacy rate, youth total (\% of people ages $15-24$ ) & 61.9 & $\ldots$ & & 76.4 & $\ldots$ & $\ldots$ & $\ldots$ \\
\hline Persistence to grade 5 , total ( $\%$ of cohort) & & & 59.7 & 61.4 & & & $\ldots$ \\
\hline Primary completion rate, total (\% of relevant age group) & 63.8 & 74.3 & 69.7 & 72.4 & 83.8 & 85.7 & $\ldots$ \\
\hline School enrollment, primary (\% net) & $\ldots$ & $\ldots$ & $\ldots$ & 78.5 & 89.4 & 88.7 & $\ldots$ \\
\hline \multicolumn{8}{|l|}{ Promote gender equality $4 /$} \\
\hline Proportion of seats held by women in national parliament (\%) & 5.0 & $\ldots$ & 7.0 & 9.0 & 9.0 & 8.3 & 8.3 \\
\hline Ratio of girls to boys in primary and secondary education (\%) & 70.3 & $\ldots$ & 82.1 & 79.8 & 90.3 & 90.5 & 90.6 \\
\hline Ratio of young literate females to males (\% ages 15-24) & 67.1 & $\ldots$ & & 80.5 & & $\ldots$ & $\ldots$ \\
\hline Share of women employed in the nonagricultural sector ( $\%$ of total nonagricultural employment'. & 12.7 & 14.4 & 16.0 & 16.8 & 17.9 & $\ldots$ & $\ldots$ \\
\hline \multicolumn{8}{|l|}{ Reduce child mortality $5 /$} \\
\hline Immunization, measles (\% of children ages $12-23$ months) & 56.0 & 72.0 & 51.0 & 55.0 & 61.0 & 64.0 & 67.0 \\
\hline Mortality rate, infant (per 1,000 live births) & 80.0 & 74.0 & 72.0 & 66.0 & $\ldots$ & 57.7 & $\ldots$ \\
\hline Mortality rate, under-5 (per 1,000$)$ & 116.6 & 104.1 & $\ldots$ & 91.2 & $\ldots$ & 76.9 & $\ldots$ \\
\hline \multicolumn{8}{|l|}{ Improved maternal health $6 /$} \\
\hline Births attended by skilled health staff (\% of total) & $\ldots$ & 34.2 & 42.3 & 42.5 & $\ldots$ & 46.6 & $\ldots$ \\
\hline Maternal mortality ratio (modeled estimate, per 100,000 live births) & $\cdots$ & $\ldots$ & $\ldots$ & $\ldots$ & $\ldots$ & $\ldots$ & $\ldots$ \\
\hline \multicolumn{8}{|l|}{ Combat HIVIAIDS, malaria, and other diseases $7 /$} \\
\hline Children orphaned by HIV/AIDS & $\ldots$ & $\ldots$ & $\ldots$ & $\cdots$ & $\ldots$ & & $\ldots$ \\
\hline Contraceptive prevalence (\% of women ages $15-49$ ) & 43.0 & $\ldots$ & $\ldots$ & 46.9 & $\ldots$ & 56.3 & $\ldots$ \\
\hline Incidence of tuberculosis (per 100,000 people) & 167.8 & $\ldots$ & $\ldots$ & $\ldots$ & $\ldots$ & $\ldots$ & $\ldots$ \\
\hline Prevalence of HIV, female (\% ages $15-24)$ & $\ldots$ & $\ldots$ & $\ldots$ & $\ldots$ & $\ldots$ & & $\ldots$ \\
\hline Prevalence of HIV, total (\% of population ages $15-49$ ) & $\ldots$ & $\ldots$ & $\ldots$ & $\ldots$ & $\ldots$ & 0.3 & ... \\
\hline Tuberculosis cases detected under DOTS (\%) & $\ldots$ & 0.3 & 1.7 & 23.4 & 55.9 & 64.5 & 67.9 \\
\hline \multicolumn{8}{|l|}{ Ensure environmental sustainability $8 /$} \\
\hline CO2 emissions (metric tons per capita) & 0.8 & 1.0 & 1.1 & 1.1 & 1.2 & & $\ldots$ \\
\hline Forest area ( $\%$ of land area) & 21.5 & 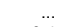 & & 22.7 & & 22.8 & \\
\hline GDP per unit of energy use (constant 2000 PPP \$ per $\mathrm{kg}$ of oil equivalent) & 3.2 & 3.4 & 3.7 & 3.9 & 4.3 & 4.5 & 4.7 \\
\hline Improved sanitation facilities (\% of population with access) & 14.0 & & $\ldots$ & 23.0 & $\ldots$ & 28.0 & $\ldots$ \\
\hline Improved water source (\% of population with access) & 71.0 & 77.0 & $\ldots$ & 82.0 & $\ldots$ & 89.0 & $\ldots$ \\
\hline Nationally protected areas (\% of total land area) & $\ldots$ & $\ldots$ & $\ldots$ & $\ldots$ & $\ldots$ & $\ldots$ & $\ldots$ \\
\hline \multicolumn{8}{|l|}{ Develop a global partnership for development $9 /$} \\
\hline Aid per capita (current US\$) & 1.6 & 1.9 & 1.6 & 1.6 & 0.6 & 1.2 & 1.2 \\
\hline Debt service (PPG and IMF only, \% of exports of G\&S, excl. workers' remittances) & & & $\ldots$ & & $\ldots$ & & \\
\hline Fixed line and mobile phone subscribers (per 1,000 people) & 0.6 & 1.3 & $\ldots$ & 3.6 & $\ldots$ & 24.3 & 24.3 \\
\hline Internet users (per 1,000 people) & & & & 0.5 & & 17.8 & 17.8 \\
\hline Total debt service (\% of exports of goods, services and income) & 31.9 & 29.7 & 21.2 & 14.7 & 13.8 & 7.7 & $\ldots$ \\
\hline Unemployment, youth female (\% of female labor force ages 15-24) & $\ldots$ & 8.0 & $\ldots$ & 10.2 & 10.8 & $\ldots$ & $\ldots$ \\
\hline Unemployment, youth male (\% of male labor force ages $15-24)$ & $\ldots$ & 8.4 & $\ldots$ & 10.1 & 10.4 & $\ldots$ & $\ldots$ \\
\hline Unemployment, youth total (\% of total labor force ages $15-24$ ) & $\ldots$ & 8.3 & $\ldots$ & 10.1 & 10.5 & $\ldots$ & $\ldots$ \\
\hline \multicolumn{8}{|l|}{ General indicators } \\
\hline Fertility rate, total (births per woman) & 4.0 & 3.6 & 3.5 & 3.2 & 2.7 & 2.7 & 2.7 \\
\hline GNI per capita, Atlas method (current US\$) & 370.0 & 380.0 & 420.0 & 460.0 & 630.0 & 820.0 & 950.0 \\
\hline GNI, Atlas method (current US\$) (billions) & 313.7 & 350.2 & 415.2 & 478.5 & 680.6 & 914.9 & $1,071.1$ \\
\hline Gross capital formation (\% of GDP) & 24.2 & 26.6 & 22.6 & 24.2 & 31.6 & 36.4 & 38.7 \\
\hline Life expectancy at birth, total (years) & 59.7 & 61.2 & 61.8 & 62.5 & 62.9 & $\ldots$ & $\ldots$ \\
\hline Literacy rate, adult total (\% of people ages 15 and above) & 48.2 & & & 61.0 & & 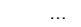 & \\
\hline Population, total (millions) & 849.5 & 932.2 & 982.2 & $1,032.5$ & $1,079.7$ & $1,109.8$ & $1,124.8$ \\
\hline Trade (\% of GDP) & 15.7 & 23.1 & 24.0 & 26.4 & 37.9 & 47.4 & 45.9 \\
\hline
\end{tabular}

Source: World Development Indicators database, November 2009.

$1 /$ In some cases the data are for earlier or later years than those stated.

2/ Halve, between 1990 and 2015, the proportion of people whose income is less than one dollar a day.

3/ Ensure that, by 2015, children everywhere, boys and girls alike, will be able to complete a full course of primary schooling.

4/ Eliminate gender disparity in primary and secondary education preferably by 2005 and to all levels of education no later than 2015.

5/ Reduce by two-thirds, between 1990 and 2015 , the under-five mortality rate.

6/ Reduce by three-quarters, between 1990 and 2015, the maternal mortality ratio.

7/ Have halted by 2015 , and begun to reverse, the spread of HIV/AIDS. Have halted by 2015, and begun to reverse, the incidence of malaria and other major diseases. 8 /ntegrate the priples of sustainable development

access to safe drinking water. By 2020, to have achieved a significant improvement in the lives of at least 100 million slum dwellers.
// Develop further an open, rule-based, predictable, non-discriminatory trading and financial system. Address the Special Needs of the Least Developed Countries. Address the Special Needs of landlocked countries and small island developing states. Deal comprehensively with the debt problems of developing countries through national and international measures in order to make debt sustainable in the long term. In cooperation with developing countries, develop and implement strategies for decent and productive work for youth. In cooperation with pharmaceutical companies, provide access to affordable, essential drugs in developing countries. In cooperation with the private sector, make available the benefits of new technologies, especially information and communications. 
Table 2. India: Selected Economic Indicators, 2005/06-2010/11 1/

\begin{tabular}{|c|c|c|c|c|c|c|}
\hline & $2005 / 06$ & $2006 / 07$ & $2007 / 08$ & $\begin{array}{r}\text { 2008/09 } \\
\text { Prel. }\end{array}$ & $\begin{array}{r}\text { 2009/10 } \\
\text { Proj. }\end{array}$ & $\begin{array}{r}\text { 2010/11 } \\
\text { Proj. }\end{array}$ \\
\hline \multicolumn{7}{|l|}{ Growth (y/y percent change) } \\
\hline Real GDP (at factor cost) & 9.5 & 9.7 & 9.0 & 6.7 & 6.7 & 8.0 \\
\hline Industrial production & 8.2 & 11.5 & 8.5 & 2.7 & $\ldots$ & $\ldots$ \\
\hline \multicolumn{7}{|l|}{ Prices (y/y percent change, average) } \\
\hline Wholesale prices (1993/94 weights) & 4.4 & 5.4 & 4.7 & 8.3 & 3.1 & 6.4 \\
\hline Wholesale prices (1993/94 weights, end of period) & 4.1 & 6.7 & 7.7 & 0.8 & 8.1 & 5.4 \\
\hline Consumer prices - industrial workers ( 2001 weights) & 4.4 & 6.7 & 6.2 & 9.1 & 11.2 & 8.8 \\
\hline \multicolumn{7}{|l|}{ Saving and investment (percent of GDP) } \\
\hline Gross saving $2 /$ & 34.3 & 35.8 & 37.6 & 37.4 & 37.5 & 38.2 \\
\hline Gross investment 2/ & 35.5 & 36.9 & 39.1 & 40.0 & 39.4 & 40.4 \\
\hline \multicolumn{7}{|l|}{ Fiscal position (percent of GDP) $3 /$} \\
\hline Central government deficit & -4.6 & -4.4 & -3.3 & -8.0 & -7.5 & -6.1 \\
\hline General government deficit & -7.2 & -6.3 & -4.7 & -11.0 & -11.1 & -9.4 \\
\hline General government debt 4/ & 84.0 & 80.8 & 78.3 & 78.2 & 80.7 & 80.6 \\
\hline \multicolumn{7}{|l|}{ Money and credit (y/y percent change, end-period) $5 / 6 /$} \\
\hline Broad money & 21.2 & 21.5 & 20.9 & 18.8 & 18.7 & $\ldots$ \\
\hline Credit to commercial sector & 32.2 & 25.8 & 20.9 & 17.0 & 11.8 & $\ldots$ \\
\hline \multicolumn{7}{|l|}{ Financial indicators (percent, end-period) $5 /$} \\
\hline 91-day treasury bill yield & 6.1 & 8.0 & 7.2 & 5.0 & 3.3 & $\ldots$ \\
\hline 10-year government bond yield & 7.5 & 8.0 & 7.9 & 7.0 & 7.2 & $\ldots$ \\
\hline Stock market (y/y percent change, end-period) & 73.7 & 15.9 & 19.7 & -37.9 & 86.2 & $\ldots$ \\
\hline \multicolumn{7}{|l|}{ External trade $7 /$} \\
\hline Merchandise exports (US\$ billions) & 105.2 & 128.9 & 166.2 & 189.0 & 157.3 & 192.1 \\
\hline y/y percent change & 23.4 & 22.6 & 28.9 & 13.7 & -16.7 & 22.1 \\
\hline Merchandise imports (US\$ billions) & 157.1 & 190.7 & 257.6 & 307.7 & 270.5 & 324.6 \\
\hline y/y percent change & 32.1 & 21.4 & 35.1 & 19.4 & -12.1 & 20.0 \\
\hline Net oil imports (US\$ billions) & 32.3 & 38.3 & 54.8 & 62.7 & 51.6 & 59.2 \\
\hline \multicolumn{7}{|l|}{ Balance of payments (US\$ billions) } \\
\hline Current account balance & -9.9 & -9.6 & -15.7 & -28.7 & -23.8 & -29.0 \\
\hline (in percent of GDP) & -1.2 & -1.0 & -1.3 & -2.5 & -1.9 & -2.1 \\
\hline Foreign direct investment, net & 3.0 & 7.7 & 15.9 & 17.5 & 15.9 & 17.4 \\
\hline Portfolio investment, net (equity and debt) & 12.5 & 7.1 & 27.4 & -14.0 & 26.1 & 19.9 \\
\hline Overall balance & 15.1 & 36.6 & 92.2 & -20.1 & 34.0 & 27.7 \\
\hline \multicolumn{7}{|l|}{ External indicators } \\
\hline Gross reserves (in billions of U.S. dollars, end-period) & 151.6 & 199.2 & 309.7 & 252.0 & 301.5 & 329.2 \\
\hline (In months of imports) $8 /$ & 7.7 & 7.7 & 10.3 & 9.3 & 9.5 & 9.3 \\
\hline External debt (percent of GDP, end-period) & 17.0 & 18.8 & 19.2 & 19.9 & 19.3 & 20.1 \\
\hline Of which: short-term debt 9/ & 3.3 & 3.8 & 7.2 & 7.8 & 7.7 & 8.4 \\
\hline Ratio of gross reserves to short-term debt (end-period) 9/ & 5.6 & 5.7 & 3.7 & 2.8 & 3.1 & 2.8 \\
\hline Gross reserves to broad money (percent; end-period) & 24.8 & 26.1 & 31.0 & 26.8 & $\ldots$ & $\ldots$ \\
\hline Debt service ratio $10 /$ & 10.1 & 4.9 & 5.3 & 5.2 & 5.3 & 6.0 \\
\hline \multicolumn{7}{|l|}{ Real effective exchange rate } \\
\hline (y/y percent change, period average for annual data) & 4.5 & -2.2 & 8.2 & -7.1 & $\ldots$ & $\ldots$ \\
\hline Exchange rate (rupee/US\$, end-period) $5 /$ & 44.6 & 43.5 & 40.1 & 50.7 & 46.4 & $\ldots$ \\
\hline \multicolumn{7}{|l|}{ Memorandum item (in percent of GDP) } \\
\hline Subsidy related bond issuance $11 /$ & 0.5 & 1.0 & 0.6 & 1.8 & 0.3 & 0.4 \\
\hline
\end{tabular}

Sources: Data provided by the Indian authorities; CEIC Data Company Ltd; Bloomberg L.P.; World Development Indicators; and Fund staff estimates and projections.

1/ Data are for April-March fiscal years.

2/ Differs from official data, calculated with gross investment and current account. Gross investment includes errors and omissions.

3/ Divestment proceeds treated as below-the-line financing. Subsidy related bond issuance included in total expenditure and in the balance.

$4 /$ Includes combined domestic liabilities of the center and the states, inclusive of MSS bonds, and sovereign external debt at year-end exchange rates

5/ For 2009/10, as of November 2009

6/ Monetary data are from CEIC.

$7 /$ On balance of payments basis.

8/ Imports of goods and services projected over the following twelve months.

9/ Short-term debt on residual maturity basis, including estimated short-term NRI deposits on residual maturity basis.

$10 /$ In percent of current account receipts excluding grants.

11 / Issued by the central government to $\mathrm{FCl}$, the state-owned oil refining/distribution companies, and fertilizer companies as compensation for losses incurred from the provision of subsidies. 
Table 3. India: Balance of Payments, 2005/06-2010/11 1/

(In billions of U.S. dollars)

\begin{tabular}{|c|c|c|c|c|c|c|}
\hline & $2005 / 06$ & $2006 / 07$ & $2007 / 08$ & $2008 / 09$ & $\begin{array}{r}\text { 2009/10 } \\
\text { Proj. }\end{array}$ & $\begin{array}{r}\text { 2010/11 } \\
\text { Proj. }\end{array}$ \\
\hline Current account balance & -9.9 & -9.6 & -15.7 & -28.7 & -23.8 & -29.0 \\
\hline Merchandise trade balance & -51.9 & -61.8 & -91.5 & -118.7 & -113.1 & -132.5 \\
\hline Merchandise exports & 105.2 & 128.9 & 166.2 & 189.0 & 157.3 & 192.1 \\
\hline Merchandise imports & 157.1 & 190.7 & 257.6 & 307.7 & 270.5 & 324.6 \\
\hline Oil & 44.0 & 56.9 & 79.7 & 93.2 & 78.7 & 92.7 \\
\hline Non-oil & 113.1 & 133.7 & 177.9 & 214.5 & 191.8 & 231.9 \\
\hline Non-factor services balance & 23.2 & 29.5 & 38.9 & 49.6 & 52.9 & 64.7 \\
\hline Receipts & 57.7 & 73.8 & 90.3 & 101.7 & 106.7 & 122.2 \\
\hline Of which: software services & 23.6 & 31.3 & 40.3 & 46.8 & $\ldots$ & \\
\hline Payments & 34.5 & 44.3 & 51.5 & 52.0 & 53.8 & 57.6 \\
\hline Income, net & -5.9 & -7.3 & -5.1 & -4.5 & -5.5 & -7.4 \\
\hline Transfers, net & 24.7 & 30.1 & 41.9 & 44.8 & 42.0 & 46.2 \\
\hline Capital account balance & 25.5 & 45.2 & 106.6 & 7.2 & 57.9 & 56.7 \\
\hline $\begin{array}{l}\text { Direct investment, net } \\
\text { Of which: direct investment in India }\end{array}$ & $\begin{array}{l}3.0 \\
8.9\end{array}$ & $\begin{array}{r}7.7 \\
22.7\end{array}$ & $\begin{array}{l}15.9 \\
34.7\end{array}$ & $\begin{array}{l}17.5 \\
35.0\end{array}$ & $\begin{array}{l}15.9 \\
28.2\end{array}$ & $\begin{array}{l}17.4 \\
30.3\end{array}$ \\
\hline Portfolio investment, net & 12.5 & 7.1 & 27.4 & -14.0 & 26.1 & 19.9 \\
\hline Government borrowing, net & 1.7 & 1.8 & 2.1 & 2.6 & 1.9 & 0.6 \\
\hline Commercial borrowing, net & 2.5 & 16.1 & 22.6 & 7.9 & 12.1 & 8.3 \\
\hline Short-term credit, net & 3.7 & 6.6 & 15.9 & -1.9 & 0.7 & 6.5 \\
\hline NRI deposits, net & 2.8 & 4.3 & 0.2 & 4.3 & 1.3 & 4.1 \\
\hline Rupee debt & -0.6 & -0.2 & -0.1 & -0.1 & -0.1 & -0.1 \\
\hline Other capital, net 2/ & -0.2 & 1.8 & 22.5 & -9.1 & 0.0 & 0.0 \\
\hline Errors and omissions & -0.5 & 1.0 & 1.3 & 1.4 & 0.0 & 0.0 \\
\hline Overall balance & 15.1 & 36.6 & 92.2 & -20.1 & 34.0 & 27.7 \\
\hline Valuation changes $3 /$ & -4.9 & 11.0 & 18.4 & -37.7 & 15.5 & 0.0 \\
\hline Increase in gross reserve stock (including valuation changes) & -10.1 & -47.6 & -110.5 & 57.7 & -49.5 & -27.7 \\
\hline \multicolumn{7}{|l|}{ Memorandum items: } \\
\hline Foreign exchange reserves & 151.6 & 199.2 & 309.7 & 252.0 & 301.5 & 329.2 \\
\hline In months of next year's imports (goods and services) & 7.7 & 7.7 & 10.3 & 9.3 & 9.5 & 9.3 \\
\hline Current account balance (percent of GDP) & -1.2 & -1.0 & -1.3 & -2.5 & -1.9 & -2.1 \\
\hline Merchandise trade balance (percent of GDP) & -6.4 & -6.8 & -7.8 & -10.3 & -8.8 & -9.6 \\
\hline Overall balance (percent of GDP) & 1.9 & 4.0 & 7.9 & -1.7 & 2.7 & 2.0 \\
\hline
\end{tabular}

Sources: CEIC Data Company Ltd; and Fund staff estimates and projections.

$1 /$ Data are for April-March fiscal years. Indian authorities' presentation.

2/ Net other capital is sum of net banking capital (RBI format) and net other capital (RBI format) less net NRI deposits.

$3 /$ Calculated as difference between the stock of reserves and the overall balance of BOP. 
Table 4. India: Reserve Money and Monetary Survey, 2005/06-2009/10 1/

\begin{tabular}{|c|c|c|c|c|c|c|c|}
\hline & \multirow[t]{2}{*}{$2005 / 06$} & \multirow[t]{2}{*}{$2006 / 07$} & \multirow[t]{2}{*}{$2007 / 08$} & \multirow[t]{2}{*}{$2008 / 09$} & \multicolumn{3}{|c|}{ 2009/10 } \\
\hline & & & & & Jun & Sep & Oct \\
\hline Reserve money & \multicolumn{7}{|c|}{ (In billions of rupees, end-period) } \\
\hline Reserve money & 5,731 & 7,090 & 8,691 & 9,880 & 9,491 & 9,652 & 9,703 \\
\hline Net domestic assets of RBI & -999 & $-1,572$ & $-3,686$ & $-2,921$ & $-3,143$ & $-3,483$ & $-3,269$ \\
\hline Claims on government (net) & 81 & 58 & $-1,682$ & 616 & 504 & 355 & 437 \\
\hline Center & 52 & 21 & $-1,682$ & 618 & 503 & 353 & 433 \\
\hline States & 30 & 36 & 0 & -2 & 2 & 2 & 5 \\
\hline Claims on commercial sector & 14 & 15 & 19 & 138 & 127 & 108 & 65 \\
\hline Claims on banks & 58 & 76 & 40 & 104 & 18 & 0 & 0 \\
\hline Other items (net) & $-1,152$ & $-1,721$ & $-2,063$ & $-3,779$ & $-3,793$ & $-3,946$ & $-3,771$ \\
\hline \multirow[t]{2}{*}{ Net foreign assets of RBI } & 6,730 & 8,662 & 12,377 & 12,801 & 12,634 & 13,135 & 12,972 \\
\hline & \multicolumn{7}{|c|}{ (Contribution to reserve money growth) } \\
\hline Reserve money & 17.2 & 23.7 & 22.6 & 13.7 & 1.9 & 0.9 & 11.8 \\
\hline Net domestic assets of RBI & 4.9 & -10.0 & -29.8 & 8.8 & 10.1 & 4.7 & 10.5 \\
\hline Claims on government (net) & 5.3 & -0.4 & -24.5 & 26.4 & 17.6 & 10.2 & 17.6 \\
\hline Net foreign assets of RBI & 12.3 & 33.7 & 52.4 & 4.9 & -8.2 & -3.9 & 1.3 \\
\hline Monetary survey & \multicolumn{7}{|c|}{ (In billions of rupees, end-period) } \\
\hline Broad money (M3) & 27,295 & 33,161 & 40,105 & 47,640 & 49,344 & 50,958 & 51,553 \\
\hline Currency with public & 4,131 & 4,829 & 5,697 & 6,664 & 6,908 & 6,934 & 7,130 \\
\hline Deposits & 23,096 & 28,257 & 34,356 & 40,921 & 42,340 & 43,983 & 44,384 \\
\hline Non-bank deposits at RBI & 69 & 75 & 53 & 56 & 96 & 41 & 40 \\
\hline Net domestic assets & 20,034 & 24,029 & 27,138 & 34,118 & 36,201 & 37,314 & 38,073 \\
\hline Domestic credit & 24,596 & 29,676 & 34,263 & 42,905 & 44,019 & 14,439 & 14,381 \\
\hline Net credit to government & 7,666 & 8,376 & 8,508 & 12,772 & 13,963 & 14,596 & 14,521 \\
\hline Of which: RBI & 81 & 58 & $-1,682$ & 616 & 504 & 355 & 437 \\
\hline Credit to commercial sector & 16,930 & 21,301 & 25,756 & 30,133 & 30,056 & -157 & -140 \\
\hline Of which: commercial bank lending & 15,071 & 19,312 & 23,619 & 27,755 & 27,776 & 28,747 & 28,872 \\
\hline Nonfood & 14,664 & 18,847 & 23,175 & 27,293 & 27,212 & 28,323 & 28,473 \\
\hline Other items (net) & $-4,562$ & $-5,647$ & $-7,125$ & $-8,787$ & $-7,817$ & 22,876 & 23,692 \\
\hline \multirow[t]{2}{*}{ Net foreign assets } & 7,262 & 9,132 & 12,967 & 13,522 & 13,143 & 13,644 & 13,481 \\
\hline & \multicolumn{7}{|c|}{ (Twelve-month percent change) } \\
\hline Broad money (M3) & 21.2 & 21.5 & 20.9 & 18.8 & 20.1 & 19.0 & 18.3 \\
\hline Net domestic assets & 25.0 & 19.9 & 12.9 & 25.7 & 31.9 & 28.0 & 24.7 \\
\hline Domestic credit & 20.7 & 20.7 & 15.5 & 25.2 & 24.2 & 22.5 & 19.3 \\
\hline Net credit to government & 1.3 & 9.3 & 1.6 & 50.1 & 49.2 & 50.9 & 46.6 \\
\hline Credit to commercial sector & 32.2 & 25.8 & 20.9 & 17.0 & 15.2 & 12.6 & 9.7 \\
\hline Of which: commercial bank lending & 37.0 & 28.1 & 22.3 & 17.5 & 15.1 & 12.7 & 9.0 \\
\hline Nonfood & 38.4 & 28.5 & 23.0 & 17.8 & 15.1 & 13.0 & 9.6 \\
\hline \multirow[t]{2}{*}{ Net foreign assets } & 11.9 & 25.7 & 42.0 & 4.3 & -3.5 & -0.4 & 3.3 \\
\hline & \multicolumn{7}{|c|}{ (Contribution to broad money growth) } \\
\hline Net domestic assets & 17.8 & 14.6 & 9.4 & 17.4 & 21.3 & 19.1 & 17.3 \\
\hline Net credit to government & 0.4 & 2.6 & 0.4 & 10.6 & 11.2 & 11.5 & 10.6 \\
\hline Of which: RBI & 1.2 & -0.1 & -5.2 & 5.7 & 4.0 & 2.3 & 3.5 \\
\hline Credit to commercial sector & 18.3 & 16.0 & 13.4 & 10.9 & 9.6 & 8.1 & 6.3 \\
\hline Net foreign assets & 3.4 & 6.9 & 11.6 & 1.4 & -1.2 & -0.1 & 1.0 \\
\hline
\end{tabular}

Sources: CEIC Data Company Ltd.; IMF International Financial Statistics; and Fund staff calculations.

$1 /$ Data are for April - March fiscal years. 
Table 5. India: Central Government Operations, 2005/06-2009/10 1/

\begin{tabular}{|c|c|c|c|c|c|c|c|}
\hline & \multirow[t]{2}{*}{$2005 / 06$} & \multirow[t]{2}{*}{$2006 / 07$} & \multirow[t]{2}{*}{$2007 / 08$} & \multicolumn{2}{|c|}{$2008 / 09$} & \multicolumn{2}{|c|}{$2009 / 10$} \\
\hline & & & & Budget & Prov. & Budget & $\begin{array}{l}\text { Staff } \\
\text { proj. }\end{array}$ \\
\hline & \multicolumn{7}{|c|}{ (In billions of rupees) } \\
\hline Net tax revenue & 2,718 & 3,532 & 4,413 & 5,090 & 4,495 & 4,767 & 4,690 \\
\hline Gross tax revenue & 3,662 & 4,735 & 5,931 & 6,877 & 6,097 & 6,411 & 6,296 \\
\hline Of which: corporate tax & 1,013 & 1,443 & 1,929 & 2,264 & 2,138 & 2,567 & 2,568 \\
\hline customs duties & 651 & 863 & 1,041 & 1,189 & 998 & 980 & 864 \\
\hline other taxes & 326 & 502 & 699 & 662 & 629 & 670 & 669 \\
\hline Less: States' share & 944 & 1,203 & 1,518 & 1,788 & 1,602 & 1,644 & 1,606 \\
\hline Nontax revenue $2 /$ & 874 & 943 & 1,479 & 1,106 & 1,102 & 1,559 & 1,409 \\
\hline Grants & 30 & 25 & 27 & 18 & 28 & 21 & 21 \\
\hline Total expenditure and net lending & 5,102 & 5,926 & 7,189 & 7,637 & 8,926 & 10,369 & 10,376 \\
\hline Capital expenditure and net lending 5 / & 557 & 624 & 1,088 & 871 & 831 & 1,194 & 1,149 \\
\hline Overall balance & $-1,480$ & $-1,426$ & $-1,269$ & $-1,423$ & $-3,301$ & $-4,021$ & $-4,255$ \\
\hline Overall balance (authorities' definition) 6/ & $-1,464$ & $-1,426$ & $-1,269$ & $-1,333$ & $-3,301$ & $-4,010$ & $-4,105$ \\
\hline Overall balance (augmented) 7/ & $-1,653$ & $-1,829$ & $-1,557$ & $\ldots$ & $-4,261$ & $-4,221$ & $-4,455$ \\
\hline Financing & 1,480 & 1,426 & 1,269 & 1,423 & 3,301 & 4,021 & 4,255 \\
\hline External (net) & 75 & 85 & 93 & 110 & 110 & 160 & 160 \\
\hline \multirow[t]{2}{*}{ Domestic (net) } & 1,405 & 1,341 & 1,176 & 1,313 & 3,191 & 3,861 & 4,094 \\
\hline & \multicolumn{7}{|c|}{ (In percent of GDP) } \\
\hline Total revenue and grants & 10.1 & 10.9 & 12.5 & 11.7 & 10.6 & 10.8 & 10.4 \\
\hline Net tax revenue & 7.6 & 8.6 & 9.3 & 9.6 & 8.4 & 8.1 & 8.0 \\
\hline Gross tax revenue & 10.2 & 11.5 & 12.6 & 13.0 & 11.5 & 10.9 & 10.7 \\
\hline Of which: corporate tax & 2.8 & 3.5 & 4.1 & 4.3 & 4.0 & 4.4 & 4.4 \\
\hline Current expenditure $3 /$ & 12.7 & 12.8 & 12.9 & 12.8 & 15.2 & 15.7 & 15.7 \\
\hline Of which: interest payments & 3.7 & 3.6 & 3.6 & 3.6 & 3.6 & 3.9 & 3.8 \\
\hline wages and salaries & 1.0 & 1.0 & 0.9 & 1.0 & 1.4 & 1.5 & 1.6 \\
\hline subsidies $4 /$ & 1.3 & 1.4 & 1.5 & 1.3 & 2.4 & 1.9 & 2.0 \\
\hline Capital expenditure and net lending $5 /$ & 1.6 & 1.5 & 2.3 & 1.6 & 1.6 & 2.0 & 2.0 \\
\hline Overall balance & -4.1 & -3.5 & -2.7 & -2.7 & -6.2 & -6.9 & -7.2 \\
\hline Overall balance (authorities' definition) 6/ & -4.1 & -3.5 & -2.7 & -2.5 & -6.2 & -6.8 & -7.0 \\
\hline Overall balance (augmented) $7 /$ & -4.6 & -4.4 & -3.4 & $\ldots$ & -8.0 & -7.0 & -7.6 \\
\hline Financing & 4.1 & 3.5 & 2.7 & 2.7 & 6.2 & 6.9 & 7.2 \\
\hline External (net) & 0.2 & 0.2 & 0.2 & 0.2 & 0.2 & 0.3 & 0.3 \\
\hline Domestic (net) & 3.9 & 3.2 & 2.5 & 2.5 & 6.0 & 6.6 & 7.0 \\
\hline Of which: market borrowing & 2.7 & 2.7 & 2.7 & 1.9 & 4.9 & 6.8 & 6.8 \\
\hline small savings (net of states' share) & 0.2 & 0.1 & -0.1 & 0.3 & 0.1 & 0.3 & 0.3 \\
\hline divestment receipts & 0.0 & 0.0 & 0.1 & 0.2 & 0.0 & 0.0 & 0.3 \\
\hline \multicolumn{8}{|l|}{ Memorandum items: } \\
\hline Primary balance & -0.4 & 0.2 & 0.9 & 0.9 & -2.6 & -3.0 & -3.4 \\
\hline Current balance 6 / & -2.5 & -1.9 & -0.4 & -1.0 & -4.6 & -4.8 & -5.0 \\
\hline Current balance (augmented) $7 /$ & -3.0 & -2.9 & -1.0 & $\ldots$ & -6.4 & -5.0 & -5.4 \\
\hline Central government debt $8 /$ & 63.0 & 61.5 & 60.1 & 57.7 & 58.9 & 59.7 & 61.2 \\
\hline Subsidy-related bonds $9 /$ & 0.5 & 1.0 & 0.7 & 0.0 & 1.8 & 0.2 & 0.3 \\
\hline Of which: Food Corporation of India bonds & 0.0 & 0.4 & 0.0 & $\ldots$ & 0.0 & 0.0 & 0.0 \\
\hline Oil bonds & 0.5 & 0.6 & 0.4 & $\ldots$ & 1.4 & 0.2 & 0.3 \\
\hline Fertilizer bonds & 0.0 & 0.0 & 0.2 & $\ldots$ & 0.4 & 0.0 & 0.0 \\
\hline Nominal GDP (in Rs. billion) & 35,867 & 41,292 & 47,234 & 53,038 & 53,218 & 58,566 & 58,725 \\
\hline
\end{tabular}

Sources: Data provided by the Indian authorities; and Fund staff estimates and projections.

$1 /$ Data for April - March fiscal years.

2/ In 2007/08, includes a special dividend payment from the RBI amounting to 0.7 percent of GDP. The authorities include this item under "other capital receipts" rather than non-tax revenue.

$3 /$ Includes the surcharge on Union duties transferred to the National Calamity Contingency Fund.

4/ Excludes subsidy-related bond issuance.

5/ In 2007/08, includes roughly 0.7 percent of GDP for the government's purchase of SBI shares from the RBI.

6/ Treats divestment as a revenue item until 2005/06 and after 2009/10 (included). In 2008/09, authorities treat proceeds from selling shares vested with SUTI as revenue in the Budget.

7/ Treats divestment receipts as a below-the-line financing item. Includes subsidy-related bond issuance as current expenditure.

8/ External debt measured at historical exchange rates. Inclusive of MSS bonds.

9/ Issued by the central government to the Food Corporation of India, fertilizer producers, and the state-owned oil refining/distribution companies as compensation for losses incurred from the subsidized provision of commodities. 
Table 6. India: General Government Operations, 2005/06-2009/10 1/

\begin{tabular}{|c|c|c|c|c|c|c|c|}
\hline & \multirow[t]{2}{*}{ 2005/06 } & \multirow[t]{2}{*}{$2006 / 07$} & \multirow{2}{*}{$\frac{2007 / 08}{\text { Prov. 2/ }}$} & \multicolumn{2}{|c|}{$2008 / 09$} & \multicolumn{2}{|c|}{$2009 / 10$} \\
\hline & & & & Budget & Prov. 3/ & Budget & Staff proj. \\
\hline & \multicolumn{7}{|c|}{ (In billions of rupees) } \\
\hline Total revenue and grants & 7,040 & 8,735 & 10,929 & 11,882 & 11,010 & 12,338 & 12,011 \\
\hline Tax revenue $4 /$ & 5,785 & 7,261 & 8,773 & 10,219 & 9,215 & 10,046 & 9,869 \\
\hline Nontax revenue $5 / 6$ / & 1,226 & 1,449 & 2,129 & 1,645 & 1,767 & 2,271 & 2,121 \\
\hline Grants & 30 & 25 & 27 & 18 & 28 & 21 & 21 \\
\hline Total expenditure and net lending $7 / 8$ / & 9,457 & 10,935 & 12,850 & 14,371 & 15,902 & 18,300 & 18,389 \\
\hline General government balance & $-2,417$ & $-2,200$ & $-1,921$ & $-2,489$ & $-4,892$ & $-5,961$ & $-6,377$ \\
\hline Financing & 2,417 & 2,200 & 1,921 & 2,489 & 4,892 & 5,961 & 6,377 \\
\hline External (net) & 75 & 85 & 93 & 110 & 110 & 160 & 160 \\
\hline Domestic (net) & 2,342 & 2,116 & 1,828 & 2,379 & 4,782 & 5,801 & 6,217 \\
\hline \multirow[t]{2}{*}{ Disinvestment receipts } & 16 & 24 & 128 & 252 & 155 & 27 & 166 \\
\hline & \multicolumn{7}{|c|}{ (In percent of GDP) } \\
\hline Total revenue and grants & 19.6 & 21.2 & 23.1 & 22.4 & 20.7 & 21.1 & 20.5 \\
\hline Tax revenue $4 /$ & 16.1 & 17.6 & 18.6 & 19.3 & 17.3 & 17.2 & 16.8 \\
\hline Nontax revenue $5 / 6$ / & 3.4 & 3.5 & 4.5 & 3.1 & 3.3 & 3.9 & 3.6 \\
\hline \multicolumn{8}{|l|}{ Grants } \\
\hline Total expenditure and net lending $7 / 8$ / & 26.4 & 26.5 & 27.2 & 27.1 & 29.9 & 31.2 & 31.3 \\
\hline General government balance & -6.7 & -5.3 & -4.1 & -4.7 & -9.2 & -10.2 & -10.9 \\
\hline (including divestment receipts) & -6.7 & -5.3 & -3.8 & -4.2 & -8.9 & -10.1 & -10.6 \\
\hline (augmented with subsidy-related bonds) & -7.2 & -6.3 & -4.7 & $\ldots$ & -11.0 & -10.4 & -11.2 \\
\hline Domestic financing (net) & 6.5 & 5.1 & 3.9 & 4.5 & 9.0 & 9.9 & 10.6 \\
\hline \multicolumn{8}{|l|}{ Memorandum items: } \\
\hline Primary balance & -1.1 & 0.3 & 1.4 & 0.7 & -3.9 & -4.6 & -5.3 \\
\hline Nondefense capital expenditure & 3.0 & 3.2 & 4.1 & 3.6 & 3.1 & 3.4 & 3.3 \\
\hline Net interest payments & 5.7 & 5.6 & 5.5 & 5.4 & 5.3 & 5.6 & 5.6 \\
\hline Central government balance & -4.1 & -3.5 & -2.7 & -2.7 & -6.2 & -6.9 & -7.2 \\
\hline State and union territory governments' balance $9 /$ & -2.5 & -1.9 & -1.5 & -2.0 & -3.1 & -3.4 & -3.7 \\
\hline Consolidation items $10 /$ & -0.1 & 0.0 & 0.1 & 0.0 & 0.1 & 0.1 & 0.1 \\
\hline Subsidy-related bond issuance & 0.5 & 1.0 & 0.6 & $\ldots$ & 1.8 & 0.2 & 0.3 \\
\hline General government debt $11 /$ & 84.0 & 80.8 & 78.3 & 78.5 & 78.2 & 79.6 & 81.7 \\
\hline
\end{tabular}

Sources: Data provided by the Indian authorities; state level data from the RBI Study on State Finances; and Fund staff amalgamate and prepare projections.

1/ The consolidated general government comprises the central government (CG) and state governments. Data for April - March fiscal years.

2/ Based on RBI's estimate of provisional outturn for state finances.

3/ Based on RBI's revised estimates of state finances.

4/ Tax revenue equals tax revenue of central government (CG), including NCCF and states' share, plus state tax revenue.

$5 /$ Nontax revenue equals nontax revenue of CG, less interest payments by states on $C G$ loans, plus nontax revenue of states.

6/ In 2007/08, includes a special dividend payment from the RBI amounting to roughly 0.7 percent of GDP. The authorities include this item under "other capital receipts."

7/ Expenditure and net lending equals total expenditure and net lending of CG (authorities' definition excluding subsidy-related bonds), less net loans and grants to states and union territories, plus total expenditure of states (excluding interest payments on CG loans).

8/ In 2007/08, includes 0.7 percent of GDP for the government's purchase of SBI shares from the RBI.

$9 /$ The authorities treat states' divestment proceeds, including land sales, above-the-line as miscellaneous capital receipts. Staff's definition treats divestment receipts as a below-the-line financing item.

10/ Above-the-line items in the CGA, which cancel out in the consolidation (e.g., loans to states).

11 / Includes combined domestic liabilities of CG and states governments, inclusive of MSS bonds, and sovereign external debt at year-end exchange rates. 
Table 7. India: Macroeconomic Framework, 2005/06-2013/14 1/

\begin{tabular}{|c|c|c|c|c|c|c|c|c|c|}
\hline & \multirow[b]{2}{*}{$2005 / 06$} & \multirow[b]{2}{*}{$2006 / 07$} & \multirow[b]{2}{*}{$2007 / 08$} & \multirow[b]{2}{*}{$2008 / 09$} & \multicolumn{5}{|c|}{ Projections } \\
\hline & & & & & $2009 / 10$ & $2010 / 11$ & $2011 / 12$ & $2012 / 13$ & $2013 / 14$ \\
\hline \multicolumn{10}{|l|}{ Growth (percent change) } \\
\hline Real GDP (at factor cost) & 9.5 & 9.7 & 9.0 & 6.7 & 6.7 & 8.0 & 7.7 & 7.8 & 8.1 \\
\hline Non-agricultural sector & 10.4 & 11.2 & 9.9 & 7.8 & 8.2 & 8.6 & 8.6 & 8.7 & 8.9 \\
\hline Real GDP (at factor cost, on calendar year basis) & 9.2 & 9.8 & 9.4 & 7.3 & 5.6 & 7.7 & 7.8 & 7.9 & 8.0 \\
\hline \multicolumn{10}{|l|}{ Prices (percent change, period average) } \\
\hline Wholesale prices (1993/94 weights) & 4.4 & 5.4 & 4.7 & 8.3 & 3.3 & 6.9 & 4.6 & 4.0 & 4.0 \\
\hline Consumer prices & 4.4 & 6.7 & 6.2 & 9.1 & 11.2 & 8.8 & 5.0 & 4.0 & 4.0 \\
\hline Interest rate on general government domestic debt (percent) & 7.8 & 8.0 & 6.7 & 7.8 & 10.2 & 9.7 & 9.1 & 9.0 & 7.0 \\
\hline \multicolumn{10}{|l|}{ Saving and investment (percent of GDP) } \\
\hline Gross saving 2/ & 34.3 & 35.8 & 37.7 & 37.5 & 37.4 & 38.2 & 39.3 & 40.2 & 41.1 \\
\hline Gross investment 3/ & 35.5 & 36.9 & 39.1 & 40.0 & 39.2 & 40.3 & 41.1 & 41.9 & 42.9 \\
\hline \multicolumn{10}{|l|}{ Fiscal position (percent of GDP) } \\
\hline Central government balance - authorities 4/ & -4.1 & -3.5 & -2.7 & -6.2 & -7.2 & -5.8 & -4.8 & -3.5 & -3.0 \\
\hline Central government balance - augmented 5 / & -4.6 & -4.4 & -3.3 & -8.0 & -7.5 & -6.1 & -5.1 & -3.8 & -3.3 \\
\hline General government balance - augmented 5/ & -7.2 & -6.3 & -4.7 & -11.0 & -11.1 & -9.4 & -8.0 & -6.4 & -5.5 \\
\hline General government debt $6 /$ & 84.0 & 80.8 & 78.3 & 78.2 & 80.7 & 80.6 & 79.8 & 77.7 & 74.7 \\
\hline \multicolumn{10}{|l|}{ External trade (percent change, BOP basis) } \\
\hline Merchandise exports (in U.S. dollar terms) & 23.4 & 22.6 & 28.9 & 13.7 & -16.7 & 22.1 & 15.5 & 12.2 & 11.2 \\
\hline Merchandise imports (in U.S. dollar terms) & 32.1 & 21.4 & 35.1 & 19.4 & -12.1 & 20.0 & 11.8 & 11.1 & 11.3 \\
\hline \multicolumn{10}{|l|}{ Balance of payments (in billions of U.S. dollars) } \\
\hline Current account balance & -9.9 & -9.6 & -15.7 & -28.7 & -23.8 & -29.0 & -26.5 & -28.5 & -31.2 \\
\hline (in percent of GDP) & -1.2 & -1.1 & -1.3 & -2.5 & -1.9 & -2.1 & -1.8 & -1.7 & -1.7 \\
\hline (in percent of GDP, calendar year basis) & -1.3 & -1.1 & -0.7 & -2.1 & -2.0 & -2.1 & -1.8 & -1.7 & -1.7 \\
\hline Foreign direct investment, net & 3.0 & 7.7 & 15.9 & 17.5 & 15.9 & 17.4 & 19.6 & 21.5 & 27.3 \\
\hline Portfolio investment, net (equity and debt) & 12.5 & 7.1 & 27.4 & -14.0 & 26.1 & 19.9 & 18.1 & 21.5 & 25.5 \\
\hline Overall balance & 15.1 & 36.6 & 92.2 & -20.1 & 34.0 & 27.7 & 31.9 & 39.3 & 52.0 \\
\hline \multicolumn{10}{|l|}{ External indicators } \\
\hline Gross reserves (in billions of U.S. dollars, end-period) & 151.6 & 199.2 & 309.7 & 252.0 & 301.5 & 329.2 & 361.2 & 400.5 & 452.5 \\
\hline (in months of imports) $7 /$ & 7.7 & 7.7 & 10.3 & 9.3 & 9.5 & 9.3 & 8.9 & 8.9 & 9.0 \\
\hline External debt (in billions of U.S. dollars, end-period) & 138.1 & 171.3 & 224.8 & 229.5 & 247.3 & 277.4 & 313.6 & 355.5 & 404.8 \\
\hline External debt (percent of GDP, end-period) & 17.0 & 18.8 & 19.2 & 19.9 & 19.3 & 20.1 & 20.8 & 21.5 & 22.2 \\
\hline Of which: short-term debt $8 /$ & 3.3 & 3.8 & 7.2 & 7.8 & 7.7 & 8.4 & 9.0 & 9.5 & 9.8 \\
\hline Ratio of gross reserves to short-term debt (end-period) 8/ & 5.6 & 5.7 & 3.7 & 2.8 & 3.1 & 2.8 & 2.7 & 2.6 & 2.5 \\
\hline Debt service (percent of current account receipts) & 10.1 & 4.9 & 5.3 & 5.2 & 5.3 & 6.0 & 7.1 & 7.7 & 7.9 \\
\hline \multicolumn{10}{|l|}{ Memorandum items (in percent of GDP): } \\
\hline Off-budget subsidy related bond issuance $9 /$ & 0.5 & 1.0 & 0.6 & 1.8 & 0.3 & 0.4 & 0.4 & 0.3 & 0.3 \\
\hline Food Corporation of India $(\mathrm{FCl})$ bonds & 0.0 & 0.4 & 0.0 & 0.0 & 0.0 & 0.0 & 0.0 & 0.0 & 0.0 \\
\hline Oil bonds & 0.5 & 0.6 & 0.4 & 1.4 & 0.3 & 0.4 & 0.4 & 0.3 & 0.3 \\
\hline Fertilizer bonds & 0.0 & 0.0 & 0.2 & 0.4 & 0.0 & 0.0 & 0.0 & 0.0 & 0.0 \\
\hline
\end{tabular}

Sources: Data provided by the Indian authorities; CEIC Data Company Ltd; and Fund staff estimates and projections.

1/ Data are for April-March fiscal years unless otherwise mentioned. Calendar year data in 2008/09 column indicate data for 2008, for instance. 2/ Differs from official data, calculated with gross investment and current account.

3/ Statistical discrepancy adjusted.

4/ Divestment proceeds are treated as revenue until 2005/06 and after 2009/10 (included); excludes subsidy related bond issuance.

$5 /$ Divestment is treated as financing; includes subsidy related bond issuance.

$6 /$ Includes combined domestic liabilities of the center and the states, inclusive of MSS bonds, and sovereign external debt at year-end exchange rates. 7/ Imports of goods and services projected over the following twelve months.

8 / Including short-term debt on contracted maturity basis, all NRI deposits, and medium and long-term debt on residual maturity basis, different from authority's definition.

9/ Issued by the central government to $\mathrm{FCl}$, the state-owned oil refining/distribution companies, and fertilizer companies as compensation for losses incurred from the provision of subsidies. 
Table 8. India: Indicators of External Vulnerability, 2004/05-2009/10 1/

\begin{tabular}{|c|c|c|c|c|c|c|c|}
\hline & $2004 / 05$ & $2005 / 06$ & $2006 / 07$ & $2007 / 08$ & $2008 / 09$ & $2009 / 10$ & 21 \\
\hline \multicolumn{8}{|l|}{ Financial indicators } \\
\hline General government debt (percent of GDP) & 86.5 & 84.0 & 80.8 & 78.3 & 78.2 & 80.7 & (Projection) \\
\hline Broad money (percent change, 12-month basis) & 12.3 & 21.2 & 21.5 & 20.9 & 19.7 & 18.7 & (November 2009) \\
\hline Private sector credit (percent change, 12-month basis) & 26.0 & 32.2 & 25.8 & 20.9 & 22.5 & 11.8 & (November 2009) \\
\hline 91 day T-bill yield (percent; end-period) & 5.3 & 6.1 & 8.0 & 7.2 & 4.8 & 3.3 & (November 2009) \\
\hline 91 day T-bill yield (real, percent; end-period) $3 /$ & -1.1 & 1.6 & 2.4 & 2.4 & -1.3 & -1.4 & (November 2009) \\
\hline \multicolumn{8}{|l|}{ External indicators } \\
\hline Exports (percent change, 12-month basis in US $\$$ ) $4 / 5$ / & 28.5 & 23.4 & 22.6 & 28.9 & 13.7 & -16.7 & (Projection) \\
\hline Export volume (percent change, 12-month basis) $5 /$ & 11.7 & 15.5 & 16.5 & 15.3 & 14.9 & 14.5 & (Projection) \\
\hline Imports (percent change, 12-month basis in US\$) 4/ 5/ & 48.6 & 32.1 & 21.4 & 35.1 & 19.4 & -12.1 & (Projection) \\
\hline Import volume (percent change, 12-month basis) 5 / & 28.0 & 20.0 & 13.5 & 13.2 & 12.8 & 12.5 & (Projection) \\
\hline Terms of trade (percent change, 12 month basis) $5 /$ & -3.7 & -5.3 & -2.9 & -3.5 & -1.5 & 0.9 & (Projection) \\
\hline Current account balance (percent of GDP) & -0.4 & -1.2 & -1.0 & -1.3 & -2.5 & -1.9 & (Projection) \\
\hline Capital and financial account balance (percent of GDP) & 4.0 & 3.1 & 5.0 & 9.1 & 0.6 & 4.5 & (Projection) \\
\hline Of which: Net portfolio investment (debt and equity) & 1.3 & 1.5 & 0.8 & 2.3 & -1.2 & 2.0 & (Projection) \\
\hline Other investment (Ioans, trade credits, etc.) & 2.1 & 1.2 & 3.3 & 5.4 & 0.3 & 1.2 & (Projection) \\
\hline Net foreign direct investment & 0.5 & 0.4 & 0.8 & 1.4 & 1.5 & 1.2 & (Projection) \\
\hline Foreign currency reserves (billions of US\$) & 141.5 & 151.6 & 199.2 & 309.7 & 252.0 & 280.8 & (November 2009) \\
\hline Official reserves (in months of imports of goods and services) & 8.9 & 7.7 & 7.7 & 10.3 & 9.3 & 9.5 & (Projection) \\
\hline Ratio of foreign currency reserves to broad money (percent) & 27.5 & 24.8 & 26.1 & 31.0 & 26.8 & 25.2 & (November 2009) \\
\hline Total short-term external debt to reserves (percent) $6 /$ & 22.7 & 17.7 & 17.6 & 27.2 & 35.9 & 32.5 & (Projection) \\
\hline Total external debt (percent of GDP) & 19.0 & 17.0 & 18.8 & 19.2 & 19.9 & 19.3 & (Projection) \\
\hline Of which: public sector debt & 7.4 & 6.6 & 6.1 & 4.9 & 5.2 & 4.9 & (Projection) \\
\hline Total external debt to exports of goods and services (percent) & 103.5 & 84.8 & 84.5 & 87.6 & 79.0 & 93.7 & (Projection) \\
\hline External interest payments to exports of goods and services (percent) & 3.7 & 3.2 & 2.7 & 3.2 & 2.9 & 2.3 & (Projection) \\
\hline External amortization payments to exports of goods and services (percent) & 21.6 & 23.5 & 16.5 & 14.5 & 14.2 & 16.4 & (Projection) \\
\hline Exchange rate (per US\$, period average) & 44.9 & 44.3 & 45.2 & 40.3 & 46.0 & 46.4 & (November 2009) \\
\hline REER (y/y change in percent; end-period) & 1.4 & 4.2 & 0.4 & 5.4 & 11.6 & 0.3 & (November 2009) \\
\hline \multicolumn{8}{|l|}{ Financial market indicators } \\
\hline Stock market index (end-period) & 6,493 & 11,280 & 13,072 & 15,644 & 9,709 & 16,926 & (November 2009) \\
\hline \multicolumn{8}{|l|}{ Foreign currency debt rating } \\
\hline Moody's Investor Services & Baa3 & Baa3 & Baa3 & Baa2 & Baa2 & Baa3 & (November 2009) \\
\hline Standard and Poor's & $\mathrm{BB}+$ & $\mathrm{BB}+$ & BBB- & BBB- & BBB- & BBB- & (November 2009) \\
\hline Fitch Ratings & $\mathrm{BB}+$ & $\mathrm{BB}+$ & BBB- & BBB- & BBB- & BBB- & (November 2009) \\
\hline
\end{tabular}

Sources: Data provided by the Indian authorities; and Fund, Information Notice System and staff estimates and projections.

1/ Data for April-March fiscal years.

2/ Latest date available or staff estimate, as noted.

3/ Equals nominal yield minus actual WPI inflation.

4/ Data for 2008/09 are on a customs basis, whereas data for previous years are on a BOP basis

$5 /$ Terms of trade including goods and services. Goods volumes are derived from partner country trade price deflators, and services volumes are derived using

U.S. CPI from the WEO database.

6/ Including short-term debt on contracted maturity basis, all NRI deposits, and medium and long-term debt on residual maturity basis, different from authorities' definition. 
Table 9. India: Indicators of Financial System Soundness, 2004/05-2008/09

\begin{tabular}{|c|c|c|c|c|c|}
\hline & $2004 / 05$ & $2005 / 06$ & $2006 / 07$ & $2007 / 08$ & $2008 / 09$ \\
\hline \multicolumn{6}{|l|}{ Measures of financial strength and performance $1 /$} \\
\hline Risk-weighted capital adequacy ratio (CAR) & 12.8 & 12.3 & 12.3 & 13.0 & 13.2 \\
\hline Public sector banks & 12.9 & 12.2 & 12.4 & 12.5 & 12.3 \\
\hline Old Private Sector Banks & 12.5 & 11.7 & 12.1 & 14.1 & 14.3 \\
\hline New Private Sector Banks & 12.1 & 12.6 & 12.0 & 14.4 & 15.1 \\
\hline Foreign banks & 14.0 & 13.0 & 12.4 & 13.1 & 15.1 \\
\hline Number of institutions not meeting 9 percent CAR & 2 & 3 & 0 & 0 & 0 \\
\hline Public sector banks & 0 & 0 & 0 & 0 & 0 \\
\hline Old Private Sector Banks & 2 & 3 & 1 & 0 & 0 \\
\hline New Private Sector Banks & 0 & 0 & 0 & 0 & 0 \\
\hline Foreign banks & 0 & 0 & 0 & 0 & 0 \\
\hline Net nonperforming loans (percent of outstanding net loans) 2/ 3/ & 1.9 & 1.2 & 1.0 & 1.1 & 1.1 \\
\hline Public sector banks & 2.0 & 1.3 & 1.1 & 1.1 & 1.0 \\
\hline Old Private Sector Banks & 2.7 & 1.7 & 1.0 & 0.7 & 0.9 \\
\hline New Private Sector Banks & 1.9 & 0.8 & 1.0 & 1.4 & 1.6 \\
\hline Foreign banks & 0.8 & 0.8 & 0.7 & 0.8 & 1.7 \\
\hline Gross nonperforming loans (percent of outstanding loans) $3 /$ & 5.2 & 3.3 & 2.5 & 2.4 & 2.4 \\
\hline Public sector banks & 5.5 & 3.6 & 2.7 & 2.3 & 2.1 \\
\hline Old Private Sector Banks & 6.0 & 4.4 & 3.1 & 2.3 & 2.4 \\
\hline New Private Sector Banks & 3.6 & 1.7 & 1.9 & 2.9 & 3.6 \\
\hline Foreign banks & 2.8 & 1.9 & 1.8 & 1.9 & 4.2 \\
\hline Number of institutions with net NPLs above 10 percent of advances & 4 & 3 & 1 & 0 & 0 \\
\hline Public sector banks & 0 & 0 & 0 & 0 & 0 \\
\hline Old Private Sector Banks & 0 & 0 & 0 & 0 & 0 \\
\hline New Private Sector Banks & 0 & 0 & 0 & 0 & 0 \\
\hline Foreign banks & 4 & 3 & 1 & 0 & 0 \\
\hline Net profit $(+) /$ loss (-) of commercial banks 4/ & 0.9 & 0.9 & 0.9 & 1.0 & 1.0 \\
\hline Public sector banks & 0.9 & 0.8 & 0.8 & 0.9 & 0.9 \\
\hline Old Private Sector Banks & 0.3 & 0.6 & 0.7 & 1.0 & 1.0 \\
\hline New Private Sector Banks & 1.1 & 1.0 & 0.9 & 1.0 & 1.1 \\
\hline Foreign banks & 1.3 & 1.5 & 1.7 & 1.8 & 1.7 \\
\hline \multicolumn{6}{|l|}{ Balance sheet structure of all scheduled banks } \\
\hline Loan/deposit ratio & 62.6 & 70.1 & 73.5 & 74.6 & 73.9 \\
\hline Investment in government securities/deposit ratio & 38.0 & 31.9 & 28.0 & 27.9 & 28.7 \\
\hline \multicolumn{6}{|l|}{ Lending to sensitive sectors (in percent of loans and advances) } \\
\hline Real estate & 12.7 & 17.2 & 18.8 & 18.4 & 17.5 \\
\hline Capital market & 1.4 & 1.5 & 1.8 & 2.5 & 1.8 \\
\hline Commodities & 0.2 & 0.1 & 0.0 & 0.1 & 0.0 \\
\hline
\end{tabular}

Source: Annual Report, and Trends and Progress of Banking in India, Reserve Bank of India.

$1 /$ Some loan classification and provisioning standards do not meet international standards.

2/ Gross nonperforming loans less provisions.

3 / Starting in 2001/02, figure includes $\mathrm{ICICl}$, formerly a large development finance institution, which merged with $\mathrm{ICICI} B a n k$ Ltd. in 2002.

$4 /$ In percent of total assets. 


\section{Appendix 1. India's Recovery in Perspective}

India's economy has rebounded strongly and the recovery is well-entrenched. According to a "heat map" of key economic indicators, India's momentum is among the strongest in the world. Unlike other major economies where indicators have moved sideways in the past couple of months, in India the heat map shows a sustained pickup in activity since June 2009. Industrial production has gathered pace rapidly since the beginning of 2009/10. Cement production and consumer durables sales have increased substantially, pointing to a recovery of private domestic demand. Exports, which remain below last year's levels, have also rebounded strongly: in sequential terms (3-month moving average saar), exports grew by 97 percent in September. Equity portfolio inflows have also increased markedly and the stock market index has soared by 80 percent since March, with the rupee appreciating by nearly 10 percent against the U.S. dollar. Along with China and Indonesia, Indian equity prices have outperformed other major Asian economies. In India, valuation ratios have rebounded, including for cyclical stocks, pointing to a domestic demand driven recovery.

\section{Assessing Growth Momentum 1/ 2/ 3/ 4/}

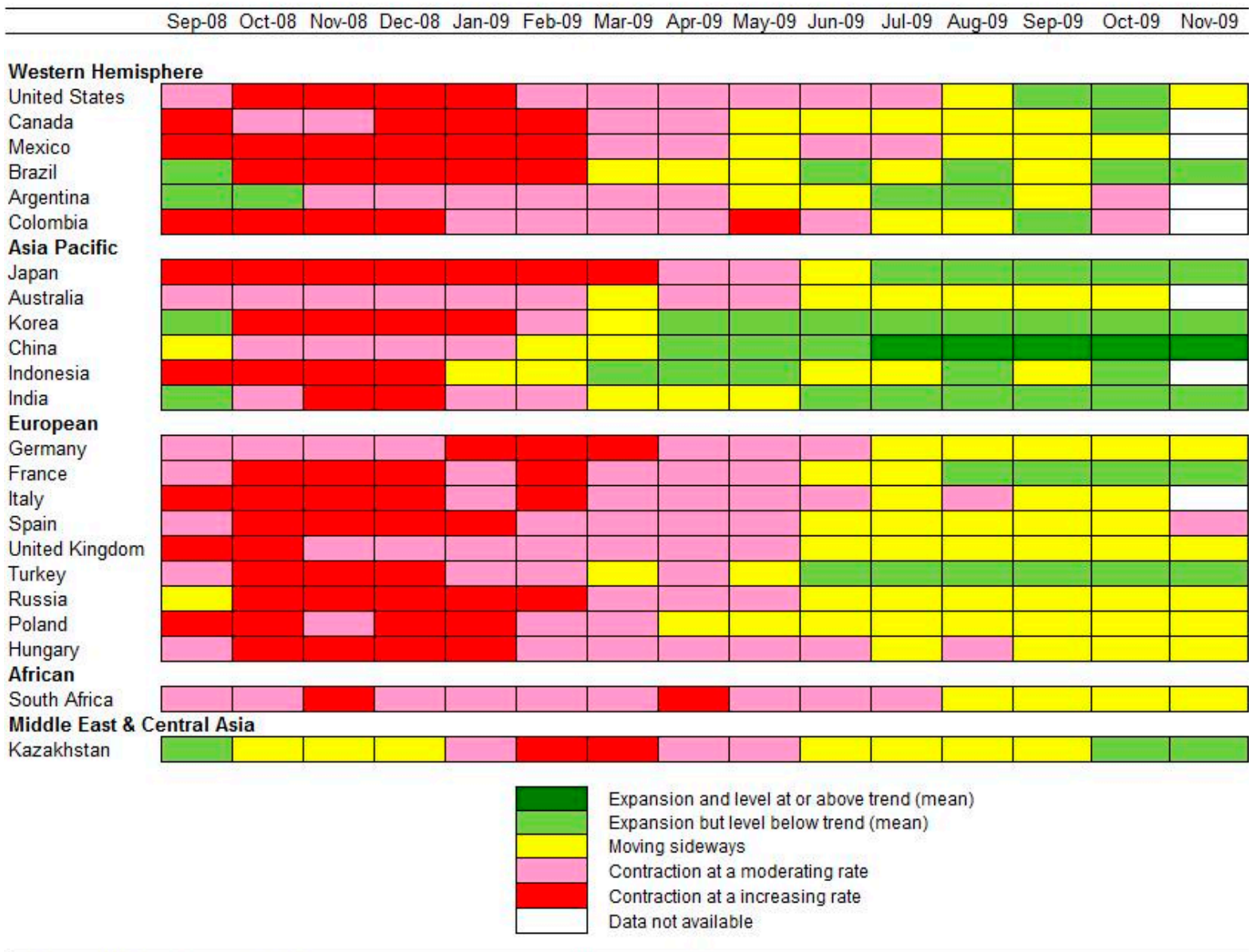

Sources: Global Data Source; Haver Analytics;Bloomberg L.P.; and staff calculations.

$1 /$ The above chart is based on the four economic indicators, including industrial production (IP), real retail sales (RS), merchandise exports (EX), and purchasing managers index (PMI).

$2 /$ Some of the ratings - particularly for recent months - are based on both actual data as well as projections of the underlying variables

3 /Saudi Arabia is not included because there is no available data.

4/Retail sales and IP are based on real data, whereas, exports are based on nominal data and PMI on survey data.

Forward looking indicators point to a continuation of these trends. The OECD's leading indicator (LI) index for India has continued to rise, approaching pre-crisis levels in September. The 
level of the index is 7 percent higher than the trough recorded in January 2009, as the decline in the LI was shallower than in other major emerging market economies (notably Russia and Brazil). India's overall PMI has stabilized at a level above the expansionary threshold since May. Although the rebound does not seem as strong as in some other emerging markets, such as Russia, where the PMI increases have continued through September, India's decline in 2008 was smaller. Further, the business expectations component of the services PMI has improved considerably since June. The export orders PMI indicates that the export growth momentum is likely to persist. The employment PMI for both manufacturing and services, also in expansionary territory, point to a strengthening of private consumption. Based on historical correlations, the manufacturing and services PMI indicate that the industry and services sectors are on track to return to close to pre-crisis growth rates in 2009Q4.
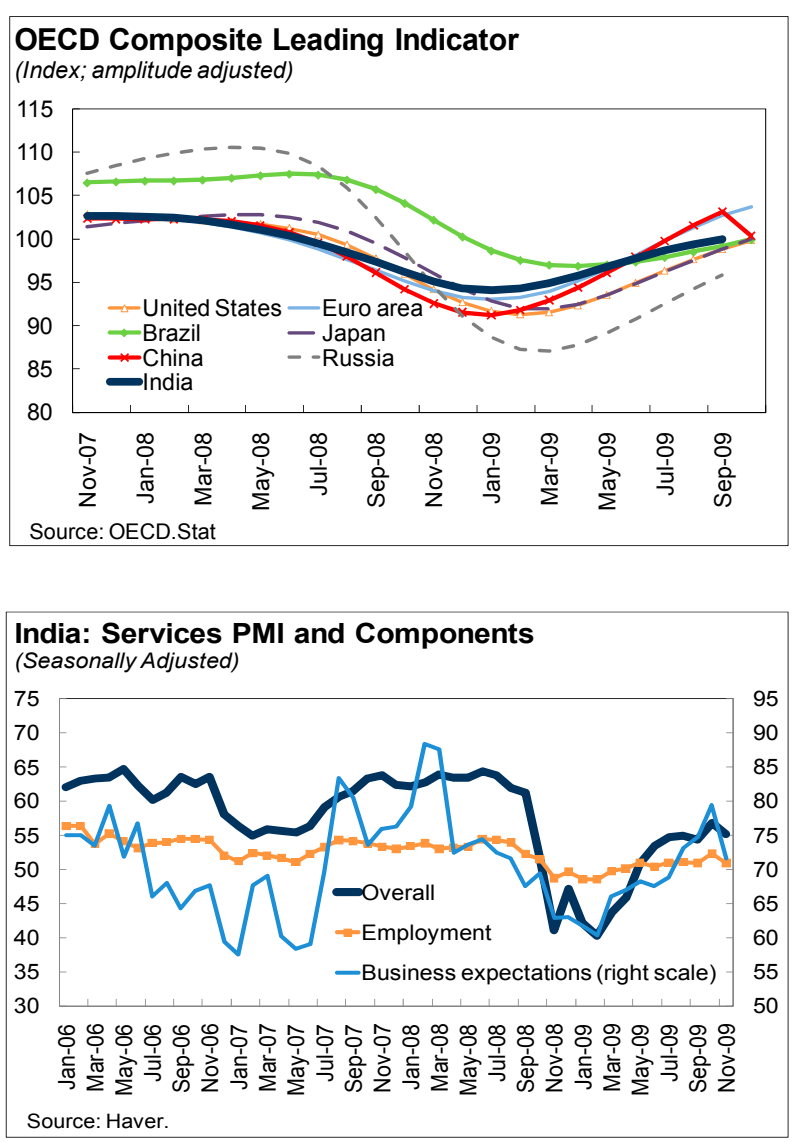
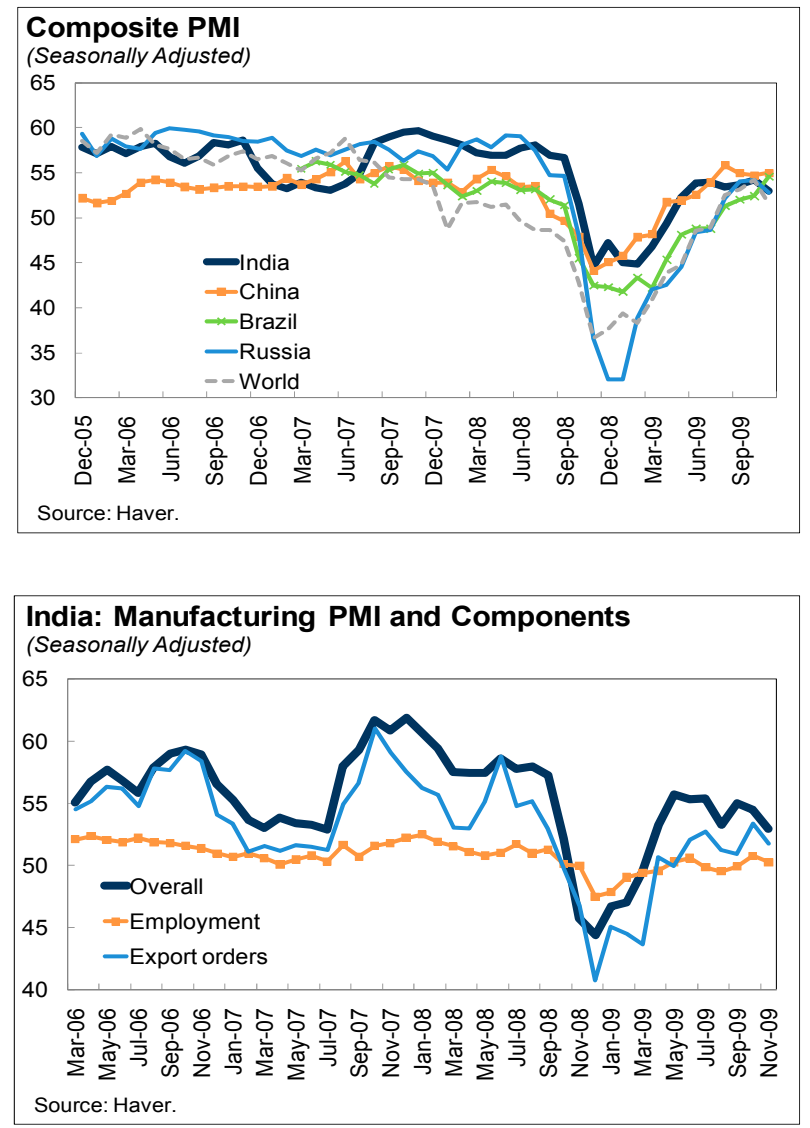


\section{Appendix 2. Economic Outlook}

Growth is projected to rise from $63 / 4$ percent in $2009 / 10$ to 8 percent the following year. While the initial impact of the global crisis on goods exports-which account for 15 percent of GDP-was significant, the main effect of the crisis was through the financial and confidence channels. ${ }^{1} \mathrm{~A}$ marked slowdown in domestic demand in late 2008-early 2009 resulted from heightened uncertainty and higher borrowing costs. But the rapid normalization of financial conditions, both globally and domestically, and improved business and consumer sentiment have led to a rebound in domestic demand since April 2009. While agricultural output is likely to contract by about 1 percent $y / y$ in $2009 / 10$ owing to deficient rainfalls, non-agricultural GDP growth is expected to gather momentum in the second half of the fiscal year, rapidly approaching potential growth. Private consumption would benefit from robust growth in disposable income and employment while investment would be boosted by rising business confidence, robust corporate profitability, and favorable financing conditions. As uncertainty about the sustainability of the recovery continues to recede, investment would accelerate significantly following a couple of quarters of strong domestic growth and stable global and domestic financing conditions. This scenario is consistent with below-trend growth in advanced economies as domestic demand would remain India's principal growth driver, but assumes low international financial market volatility and a positive outlook for corporate funding and capital flows. Preliminary evidence from fiscal multipliers also suggests that GDP growth would benefit from the direct impact of government spending (e.g., the implementation of the $6^{\text {th }}$ Pay commission by the states and other public sector entities) and the delayed impact of tax cuts on investment.

India: Summary of Medium-Term Macroeconomic Framework, 2007/08-2014/15

\begin{tabular}{|c|c|c|c|c|c|c|c|c|}
\hline & \multirow[b]{2}{*}{$2007 / 08$} & \multirow[b]{2}{*}{$2008 / 09$} & \multicolumn{6}{|c|}{ Proj. } \\
\hline & & & $2009 / 10$ & $2010 / 11$ & $2011 / 12$ & $2012 / 13$ & $2013 / 14$ & $2014 / 15$ \\
\hline \multicolumn{9}{|l|}{ Real sector (percent change) } \\
\hline Real GDP growth & 9.0 & 6.7 & 6.7 & 8.0 & 7.7 & 7.8 & 8.1 & 8.1 \\
\hline Real GDP growth, agriculture & 4.9 & 1.6 & -0.6 & 4.7 & 2.7 & 2.7 & 2.7 & 2.7 \\
\hline Real GDP growth, nonagriculture & 9.9 & 7.8 & 8.2 & 8.6 & 8.6 & 8.7 & 8.9 & 8.9 \\
\hline Inflation (period average) & 4.6 & 8.3 & 3.1 & 6.4 & 4.6 & 4.0 & 4.0 & 4.0 \\
\hline Inflation (end of period) & 7.7 & 0.8 & 8.1 & 5.4 & 4.0 & 4.0 & 4.0 & 4.0 \\
\hline \multicolumn{9}{|c|}{ Saving and investment (in percent of GDP) } \\
\hline Gross domestic investment & 39.1 & 40.0 & 39.2 & 40.3 & 41.1 & 41.9 & 42.9 & 43.9 \\
\hline Gross national saving & 37.6 & 37.4 & 37.4 & 38.2 & 39.3 & 40.2 & 41.1 & 42.3 \\
\hline Current account (in percent of GDP) & -1.3 & -2.5 & -1.9 & -2.1 & -1.8 & -1.8 & -1.8 & -1.8 \\
\hline
\end{tabular}

Source: IMF staff estimates.

Growth is projected to revert to its potential trend in 2010/11. By then, exports should return to positive growth, although the net export contribution is expected to be small as the pick up in investment will boost imports. As a result of the recovery in both exports and imports, the current account deficit is projected to widen slightly to 2.1 percent of GDP in 2010/11 from 1.9 percent of GDP in 2009/10. From a saving-investment perspective, gross national savings are expected to continue to increase, as fiscal consolidation takes hold and firms' profits boost corporate savings. Private investment would also continue to rise as infrastructure spending takes off.

\footnotetext{
${ }^{1}$ In the three-year period prior to the crisis, annual export growth averaged 25 percent, but the contribution of net exports to GDP growth was less than one percentage point.
} 
India: Conjunctural Developments

A robust economic recovery is underway and inflation has picked up.

In recent quarters, GDP growth has been supported by countercyclical policies...

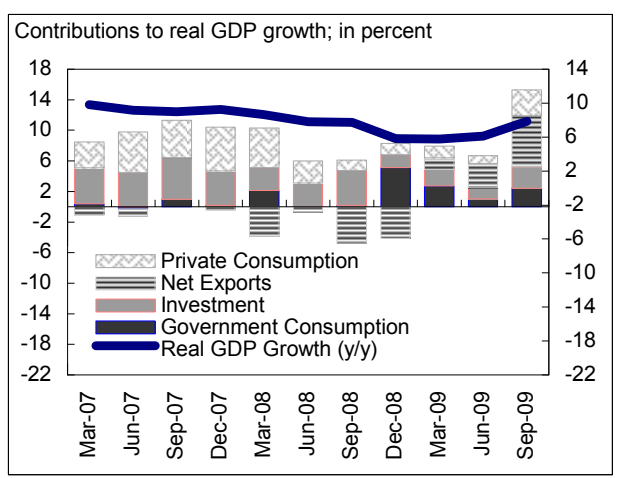

While non-food credit growth has yet to pick up..

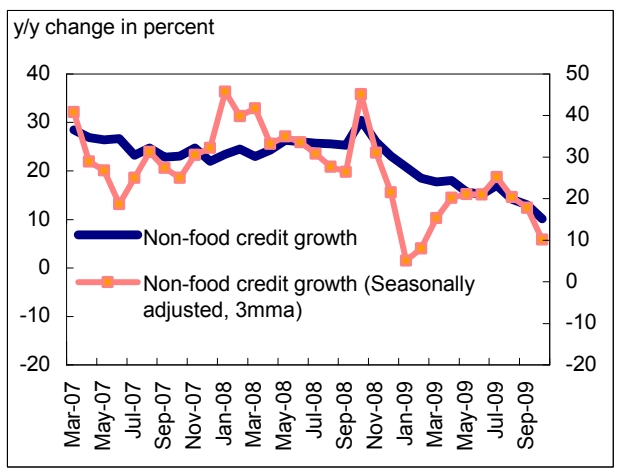

Exports are down from last year levels, but low oil prices have reduced the trade deficit.

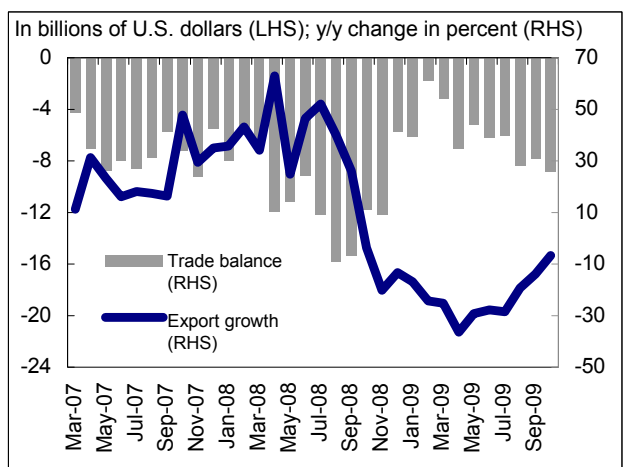

...but high frequency indicators of activity point to a broader recovery.

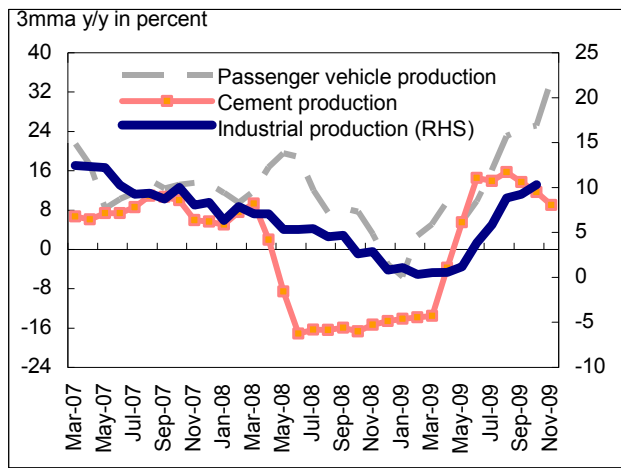

...inflation has been rising.

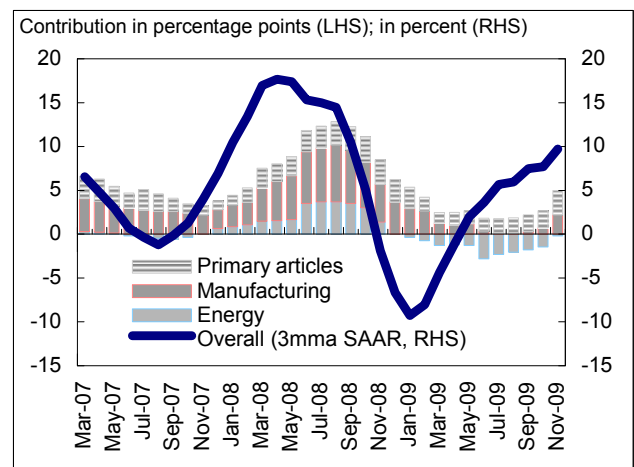

Capital inflows have surged and reserves have risen.

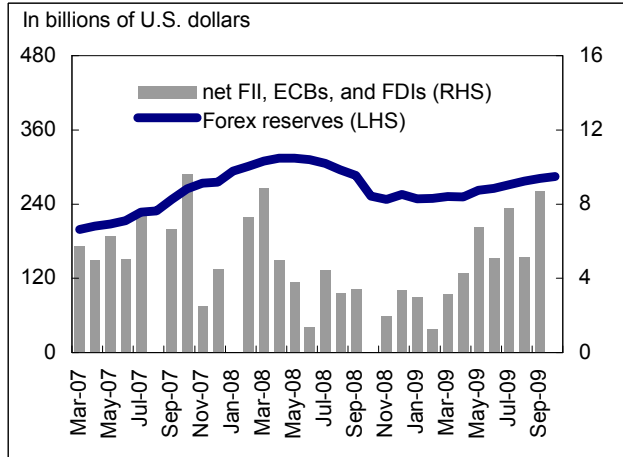

Sources: Reserve Bank of India; CEIC Data Company Ltd.; Bloomberg L.P.; and Fund staff calculations. 


\section{Appendix 3. Outlook for Capital Inflows}

After large outflows in the wake of the Lehman demise, capital flows to emerging markets (EMs) have rebounded strongly. Portfolio flows and cross-border syndicated loans explain the volatility observed. Likewise, India has seen an inflow in equity portfolio (FII) flows of \$15 billion in the year since April against an outflow of $\$ 5$ billion in the previous two quarters. External commercial borrowing (ECB) by Indian corporates has also resumed, though it has not reached pre-crisis levels.
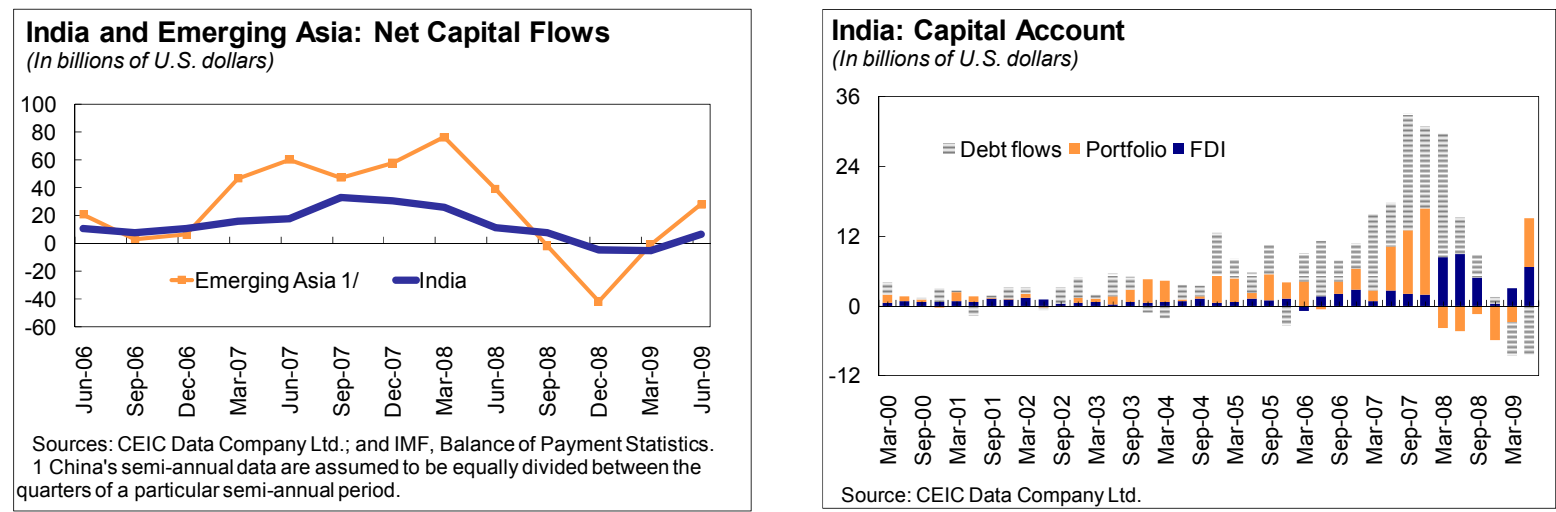

An empirical analysis of capital flows to India captures their determinants and dynamics fairly well. Using a time series model, we investigate the determinants of FII and ECB flows. The results suggest that:

- A decline in global risk aversion (proxied by the U.S. VIX) increases FII flows to India, while the coefficients on the lagged stock market index imply that investors seek markets showing positive momentum but withdraw as valuations rise;

- ECBs are also influenced by the VIX, but are more sensitive to market capitalization and growth differentials;

- Exchange rate volatility discourages both FII and ECB flows; and

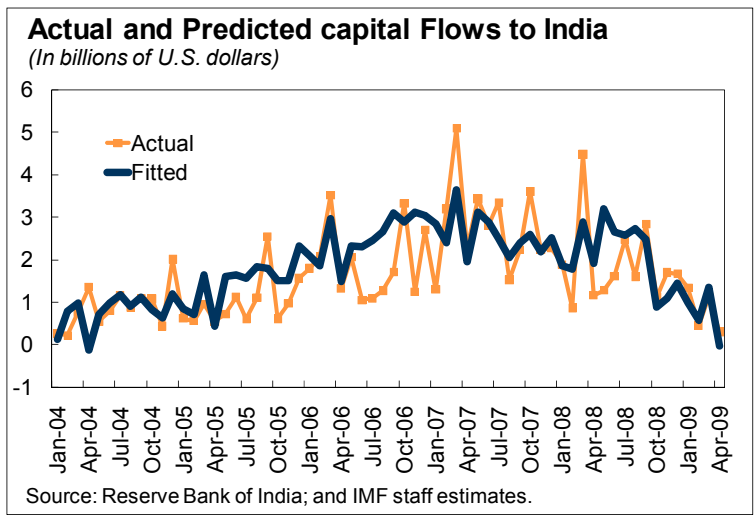

- Interest rate differentials were not found to be a significant determinant.

The outlook for capital flows to EMs and to India is positive. Historically, a low interest rate environment in advanced economies and strong growth in EMs have provided a powerful boost to capital flows to EMs. Market surveys show that global funds are underweight EMs, providing scope for a continued stock adjustment. The IIF is projecting net private flows to Emerging Asia to rise to $\$ 273$ billion in 2010 from a low of $\$ 171$ billion in 2008 , led by equity flows. For India, our estimates indicate that a sustained lower VIX could fuel about another $\$ 10$ billion in FII and ECB flows over a year. However, dedicated EM equity funds are already overweight on India, suggesting that capital flows to India will depend on the continuation of the strong domestic recovery, as well as on global financial conditions.

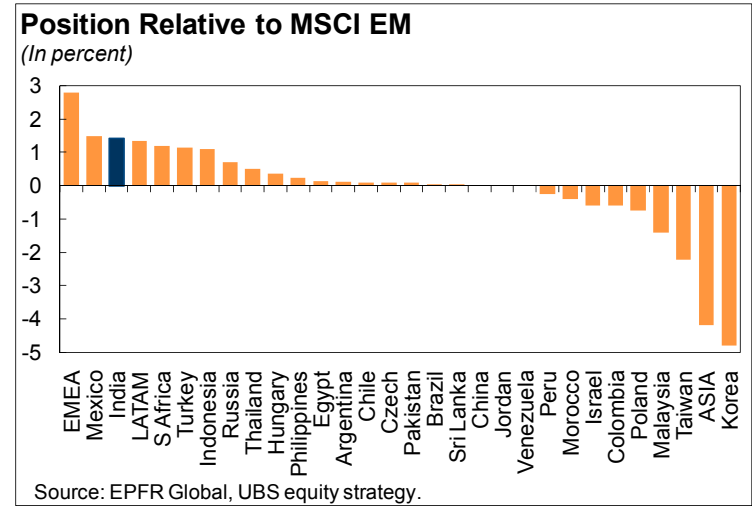




\section{Appendix 4. Inflation: Developments and Outlook}

Inflation is rising rapidly. After plummeting between October 2008 and August 2009, WPI inflation turned positive in September and is currently running at almost 5 percent $y / y$. In sequential terms WPI inflation has accelerated to about 10 percent sequentially. While food prices can partly explain the recent increase, ${ }^{1}$ manufactured goods inflation reached 4 percent $y / y$ in November and core inflation has risen steadily since June, with most indicators of underlying inflation currently above the RBI medium-term objective of 3 percent. Given the higher weight on food items, all four CPIs have hovered around double digits since August 2008. India's inflation has been one of the highest in Asia in the past few months.

Inflation expectations have also increased and the output gap is estimated to be small. The RBl's inflation expectations survey shows that households expect inflation to increase over the next three-month as well as one-year horizons. The distribution of expected WPI inflation (for 2010/11) from Consensus Forecast has also moved, with market participants expecting higher and more volatile inflation for 2010/11. Firms are regaining pricing power as evidenced by the rising ratio of output to input prices in PMI surveys. The output gap is estimated to be less than one percent, and is expected to close at the end of

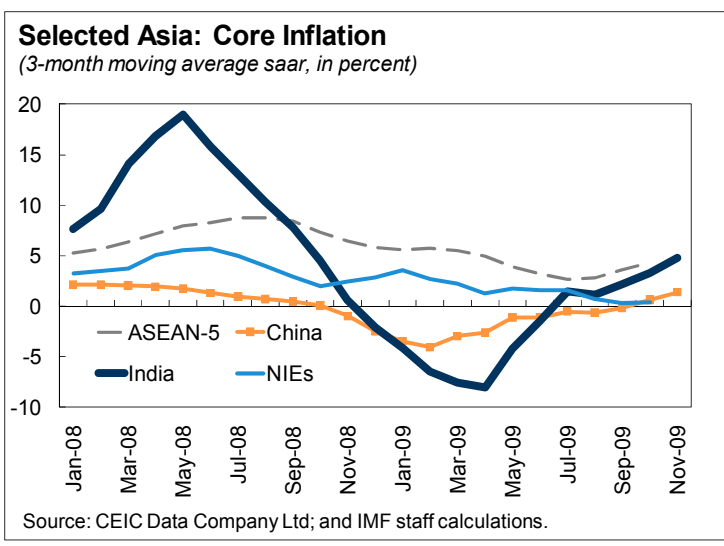

Distribution of Inflation Expectations

(2010/11 average WPI inflation, in percent)

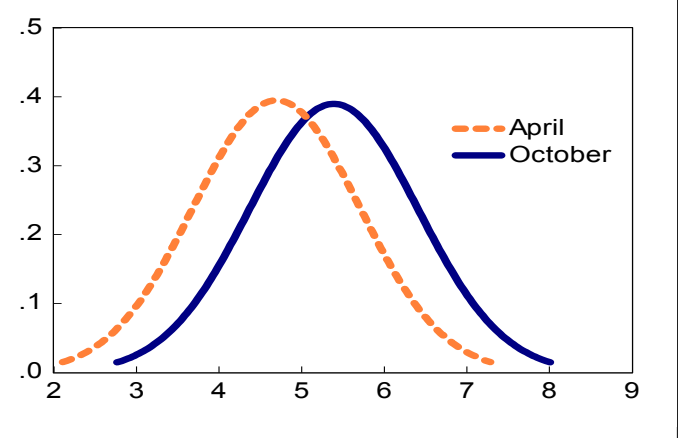
2010/11.

Inflation will peak in early $2010 / 11$ but is likely to remain elevated over the near term. Headline WPI inflation is projected to reach 8 percent $y / y$ by March 2010, and decline gradually to 5-6 percent $\mathrm{y} / \mathrm{y}$ by March 2011 because of lower food price inflation as the effects of the deficient monsoon wane.

\footnotetext{
${ }^{1}$ Food prices in India rose less than international food prices in 2007-08 given price controls and other government interventions. However, in 2009 while global food prices declined, domestic prices have been catching up, and were later affected by the drought.
} 


\section{Appendix 5. Monetary Conditions, Corporate Funding, and Asset Prices}

In its October Review, the RBI kept policy rates unchanged but began the first phase of exit. The special refinancing facilities for banks and for on-lending to mutual funds and non-bank finance companies (NBFCs) were revoked with immediate effect and the statutory liquidity requirement (SLR) was restored to its pre-crisis level of 25 percent (from 24 percent). The RBI stressed that the "current monetary stance is not the steady state and we need to reverse the expansionary stance," a clear signal that the tightening cycle has begun. The RBI also increased provisioning requirements on commercial real estate and total provisions to 70 percent of nonperforming assets (NPAs) by September 2010.

\section{Monetary conditions remain accommodative}

- $\quad$ Liquidity in the money market has remained abundant since December 2008, as the RBI has been consistently absorbing large amounts from banks. Banks have also deposited substantial funds with Mutual Funds. The various windows opened by the RBI during the crisis have been little used, and, in contrast to advanced economies, the overall expansion of the RBI balance sheet has been normal, with the injections via higher credit to government offset by the decline in net foreign assets.

- $\quad$ Short-term interest rates are low. After

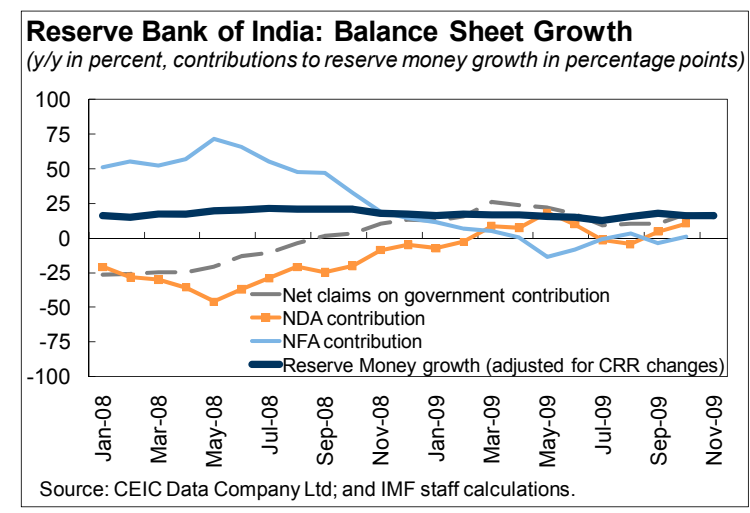
cutting the reverse repo rate by 275 basis points (bps) and the repo rate by 425 bps, the RBI has kept them unchanged at 3.25 percent and 4.75 percent, respectively, since April 2009. The call money rate has been hovering at the bottom of the policy corridor. Interest rates on bank deposits and loans, which rose in the initial phase of the crisis, have declined since the beginning of 2009. Given projected inflation, deposit rates are negative in real terms. The market is anticipating a significant tightening in policy rates and the yield curve has steepened since January.

- $\quad$ The rupee remains below its pre-crisis peak. The exchange rate appreciation since October has tightened monetary conditions slightly. In real effective terms, the rupee is broadly in line with fundamentals. ${ }^{1}$ While this suggests that there might be limited scope for further real appreciation, the impact of a stronger NEER on the REER would be mitigated by a reduction in the inflation differential. $A$ gradual real appreciation is unlikely to

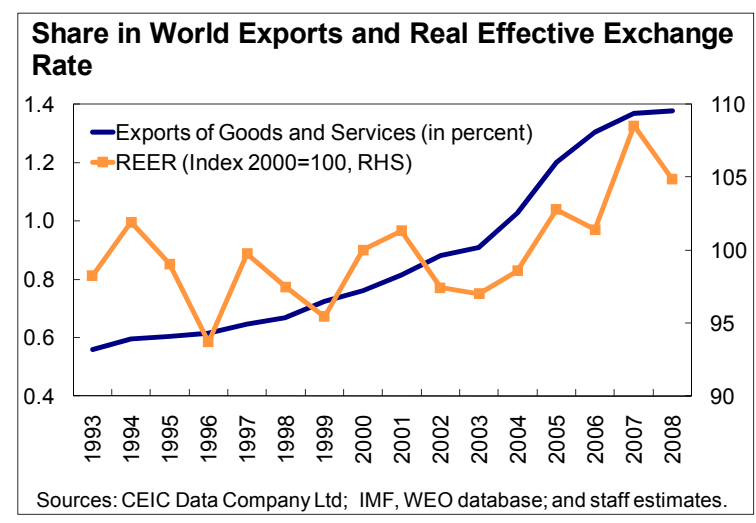

\footnotetext{
${ }^{1}$ According to CGER, the rupee is estimated to be between zero to 10 percent below its equilibrium level. The Equilibrium Real Exchange Rate approach yields an undervaluation given the depreciation over the past year, and the External Sustainability approach indicates no misalignment.
} 
pose a major challenge to exporters given the small elasticity of exports to the REER. The experience since the 1990s has also shown that trend appreciation (driven by productivity gains) has coincided with rising export shares in the world market.

\section{Asset prices are gaining momentum and corporate funding conditions have normalized.}

- $\quad$ Equity prices have surged and housing prices have staged a rebound. Equity valuation ratios have also continued to improve. The PE ratio of the Sensex is at 22, in line with its level in March 2008. The PE ratio for realty companies has risen four-fold since April 2009 and credit to real estate was up 41 percent in August. Anecdotal evidence suggests that residential real estate prices in some areas are back to October 2008 levels. Also, commercial real estate has stabilized, but the recovery is still in a nascent phase.

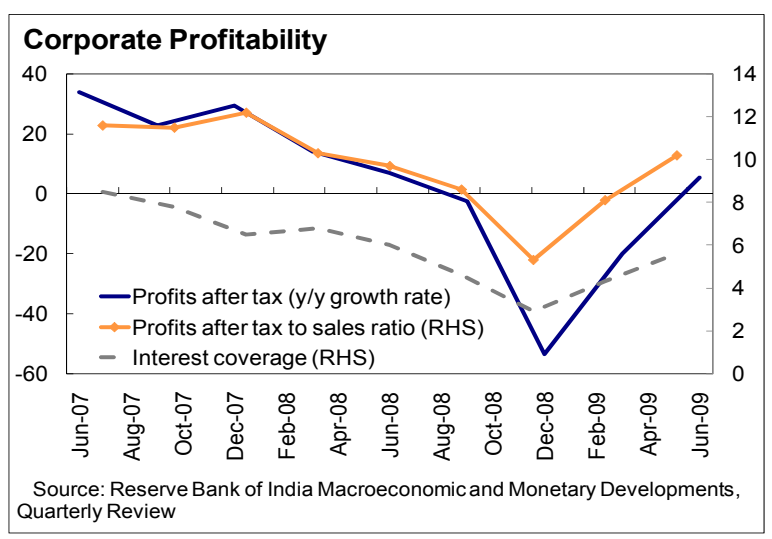

- $\quad$ Financing conditions for corporates have normalized much faster than expected and profits have grown since the onset of the crisis. Funding from nonbank sources has surpassed $2008 / 09$ levels_-issuance in the equity market has resumed and the pipeline is strong; the domestic corporate debt market has also seen increased activity. FDI and ECBs are reviving, though they are not yet at pre-crisis levels. Bank credit growth has moderated to 10 percent $y / y$ reflecting a high base and balance sheet consolidation by private banks after a strong credit cycle. ${ }^{2}$ The overall funding envelope for corporates is still likely to expand in 2009/10, as banks report substantial increases in loan approvals.

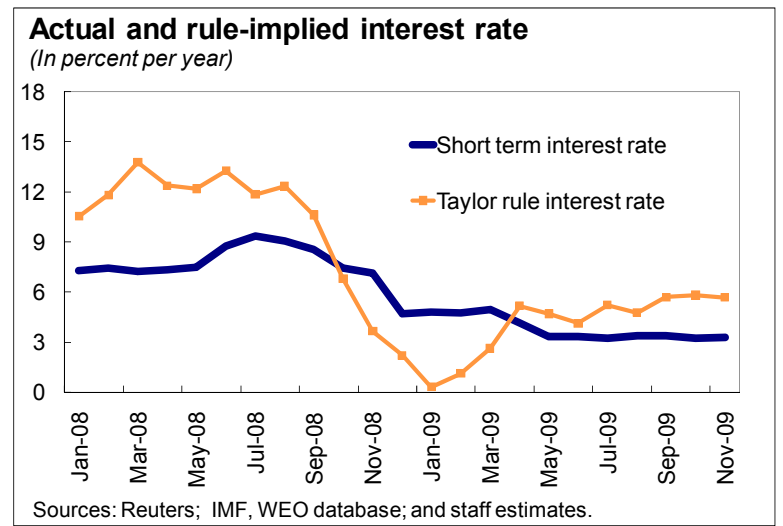

Monetary transmission lags are relatively long. The peak effect of interest rates on output is estimated at 4-6 quarters, though likely shorter in a tightening cycle. ${ }^{3}$ Currently, the policy rate is some 200 bps below the neutral rate (estimated using a simple Taylor rule). Given the monetary

\footnotetext{
${ }^{2}$ In fact, a simple econometric test for India based on quarterly data for 1996-2009 indicates the real GDP growth precedes credit growth. Shocks to activity only have a significant impact on credit after 4 quarters. This finding of a "creditless" recovery is broadly consistent with the cross-country evidence reviewed in the April 2009 WEO (Box 3.2).

${ }^{3}$ Loan repricing is faster as loans are mostly linked to the benchmark prime lending rate, while deposits are fixed term, with the typical maturity being 1 year. Also in an easing cycle, the transmission mechanism is impaired by the small savings schemes, whose interest rates are administratively set at around 8 percent.
} 
transmission lags and inertial behavior in short term interest rates, a timely and gradual normalization of the monetary stance could ensure that inflation expectations remain well anchored.

International spillovers of monetary tightening in India will likely be small. The direct spillovers should affect only countries in the region, particularly those pegged to the rupee. Indirect spillovers via growth are expected to be negligible because the RBI would only tighten once the recovery is entrenched and the monetary policy stance would return to neutral only gradually. 
India: Monetary Developments

\section{Inflation has risen and monetary policy remains accommodative.}

Inflation has picked up, driven in part by food prices.

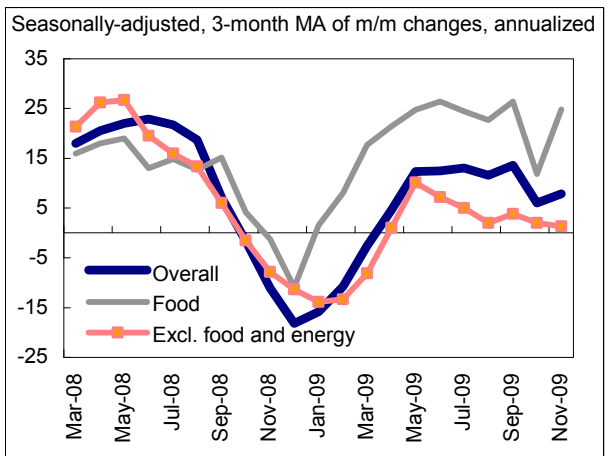

The Reserve Bank of India has consistently absorbed large amounts of liquidity...

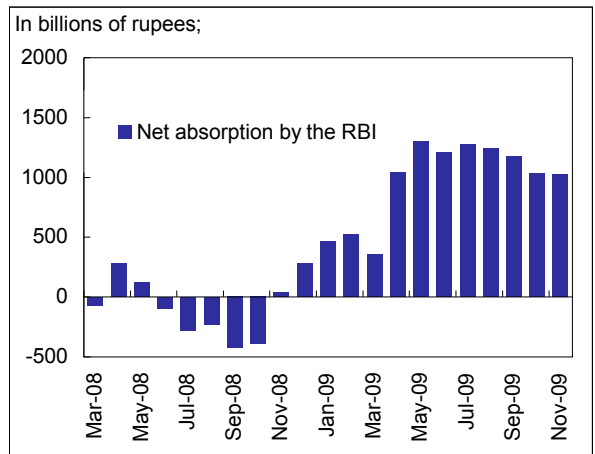

Lending and deposit rates have declined gradually...

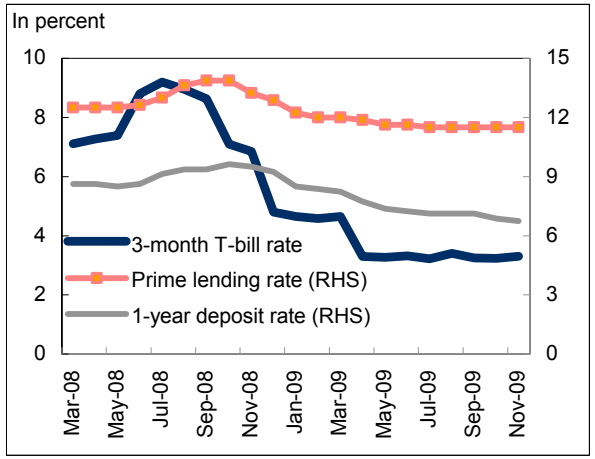

Meanwhile, monetary conditions have remained accommodative.

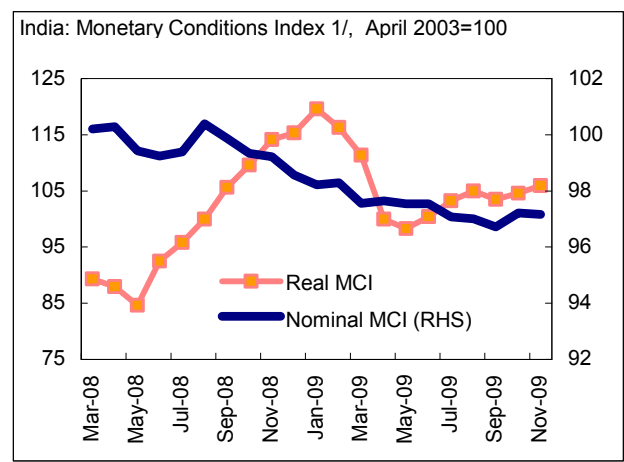

....and the call money rate has hovered at the bottom of the policy corridor.

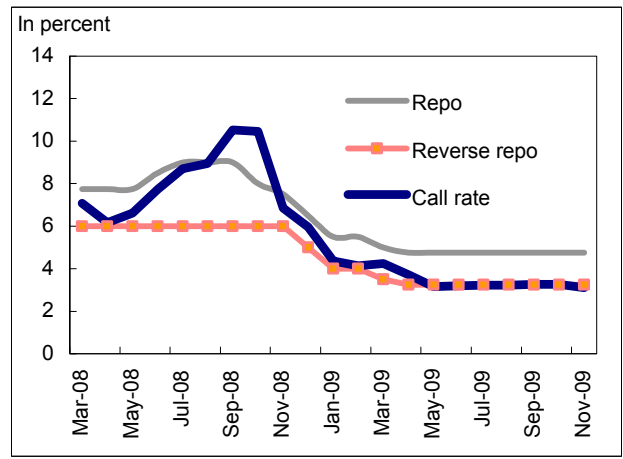

...while broad money has been steady.

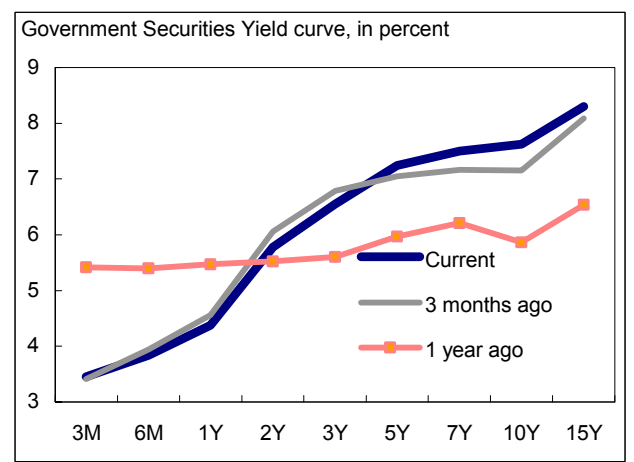

Sources: Reserve Bank of India; Bloomberg L.P.; and Fund staff calculations.

1 / The $\mathrm{MCl}$ is based on the prime lending rate, the $3 \mathrm{mma}$ of core inflation, and the REER. 


\section{Appendix 6. Technical Note on DSGE Model ${ }^{1}$}

Staff have developed a dynamic stochastic general equilibrium (DSGE) model with macrofinancial linkages for India. The model-estimated on ten key macroeconomic variables from 1996 to 2008 using Bayesian estimation techniques-incorporates the financial accelerator mechanism in a setting where firms can borrow in domestic and foreign currency. The increased reliance of Indian corporates on financing external to the firm suggests that the financial accelerator might play an important role in the propagation of shocks.

The model yields plausible estimates for the parameters of interest. The estimated elasticity of corporates' external finance premium is in line with studies for other EMs. Tests of the model suggest that it provides a good fit, especially with the inclusion of a financial accelerator. The model also outperforms empirical Bayesian VARs, providing further support for its use for policy analysis.

Estimates of the monetary policy reaction function suggest that the RBI places a relatively high weight on stabilizing the rate of depreciation of the nominal exchange rate and inflation. The results suggest that a 1 percent depreciation of the nominal exchange rate causes short-term interest rates to rise by $100 \mathrm{bps}$, while a 1 percentage point increase in inflation leads to a $90 \mathrm{bps}$ increase in rates.

Impulse response functions confirm the importance of the financial accelerator. In addition to the decline in domestic demand and appreciating exchange rate following a contractionary monetary policy shock, high interest rates and low inflation increase the real borrowing cost for corporates, decreasing investment and capital. As the price of capital falls, corporates' net worth-the value of their collateral-declines, raising the cost of borrowing further, and putting additional pressure on output. Similarly, a shock to corporates' risk premia reduces investment and capital, which via the financial accelerator increases the cost of borrowing even further.

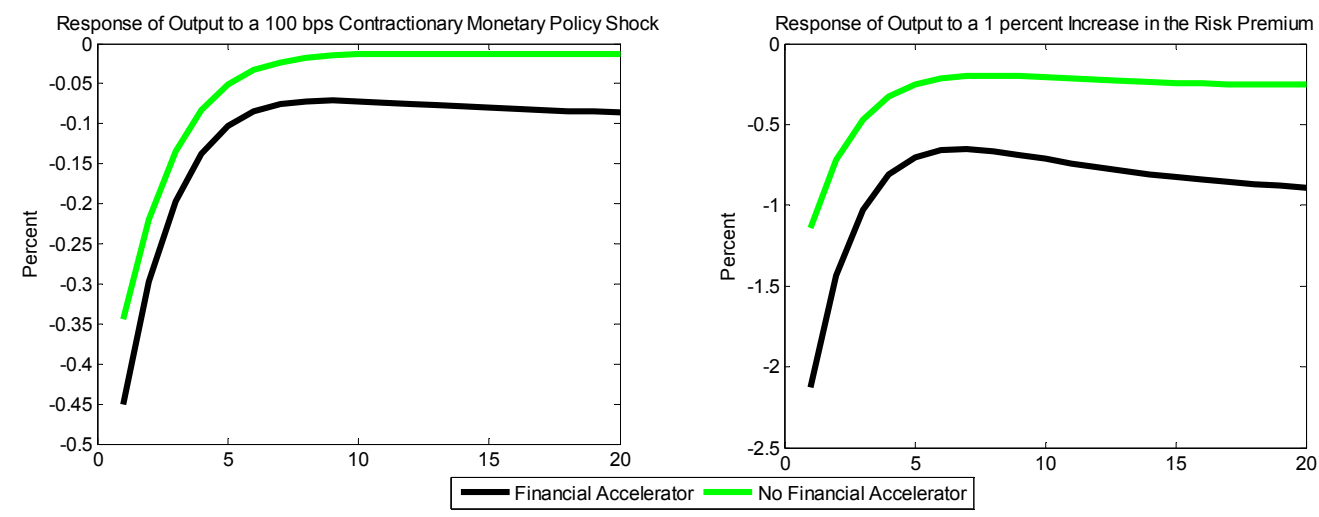

The model suggests that financial shocks are nearly as important as either demand or supply shocks. Domestic demand and supply shocks account for about 30 percent of output volatility each, compared to 25 percent for financial shocks, and 14 percent for foreign demand shocks.

\footnotetext{
${ }^{1}$ Anand, R., S. Peiris, and M. Saxegaard. "An Estimated Model with Macrofinancial Linkages for India," IMF Working Paper No. 10/21.
} 


\section{Appendix 7. Financial Stability of the Indian Banking System}

\section{India's financial soundness indicators (FSI) compare well internationally, although asset quality is deteriorating.}

The authorities' assessment. The authorities' Report of the Committee on Financial Sector Assessment (CFSA, March 2009) concluded that India's financial system was sound. Though nonperforming assets (NPAs) could increase during the slowdown, the rise was not likely to pose systemic risks. India was also found compliant with most standards and codes, with gaps noted in the implementation of bankruptcy proceedings.

\section{International} comparison of FSI and market indicators. Indian banks have an average CAR of 13 percent (tier-one capital at 9 percent) and the government has embarked on a program to boost the capital of public banks to 12
India: Finanical Soundness Indicators Compared to Other Emerging Market and Developed Economies

\begin{tabular}{lcccccc}
\hline & $\begin{array}{c}\text { Bank Regulatory } \\
\text { Capital to Risk- } \\
\text { Weighted Assets }\end{array}$ & $\begin{array}{c}\text { Bank Capital } \\
\text { to Assets }\end{array}$ & $\begin{array}{c}\text { NPLs to } \\
\text { Nonperforming } \\
\text { loans }\end{array}$ & $\begin{array}{c}\text { Bank Provisions to } \\
\text { Nonperforming } \\
\text { loans }\end{array}$ & $\begin{array}{c}\text { Bank Return } \\
\text { on Assets }\end{array}$ & $\begin{array}{c}\text { Bank Return } \\
\text { on Equity }\end{array}$ \\
\hline India & $\mathbf{1 3 . 0}$ & $\mathbf{6 . 4}$ & $\mathbf{2 . 3}$ & $\mathbf{5 2 . 6}$ & $\mathbf{1 . 0}$ & $\mathbf{1 2 . 5}$ \\
Brazil & 18.5 & 9.2 & 4.3 & 157.3 & 1.1 & 11.6 \\
China & 12.0 & 5.4 & 1.8 & 116.4 & 1.0 & 17.1 \\
Japan & 13.4 & 3.6 & 1.7 & 25.5 & 0.2 & -6.9 \\
Korea & 12.9 & 9.5 & 1.5 & 125.3 & 0.5 & 7.1 \\
Mexico & 15.2 & 9.1 & 3.8 & 143.7 & 1.2 & 12.7 \\
Russia & 18.5 & 13.6 & 7.6 & 90.8 & 0.5 & 3.6 \\
Thailand & 13.8 & 9.5 & 5.7 & 97.9 & 1.0 & 7.3 \\
\hline
\end{tabular}

Source: IMF, Global Financial Stability Report, October 2009

percent. At 2.3 percent of total assets, NPAs compare well internationally, but this comparison is complicated by the restructuring allowed in October 2008-June 2009, under which 2.9 percent of loans were rescheduled. Moody's and Crisil anticipate that gross NPAs will rise to around 5 percent by March 2011, similar to the staff's baseline. Provision coverage is low, but the RBI has recently instructed banks to raise it to 70 percent by September 2010. Profitability is broadly in line with other EM and is holding up well. Market indicators of financial stability also paint a relatively healthy picture of Indian banks.

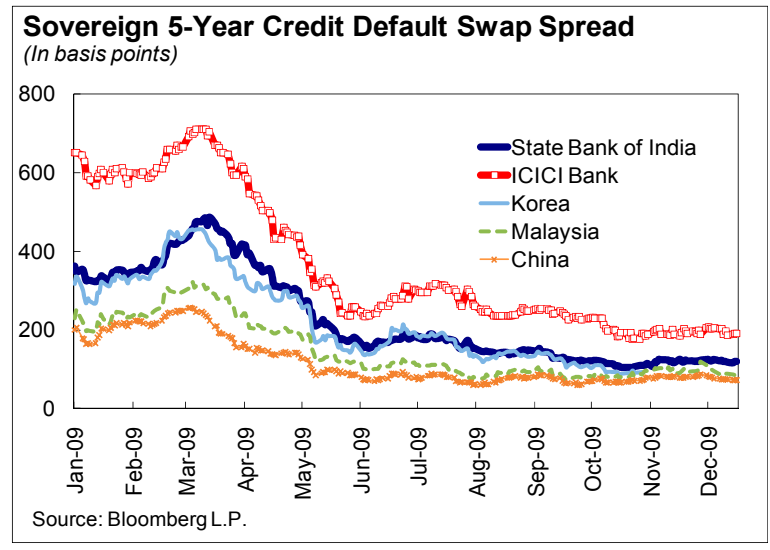

India: Expected Default Frequency of Major Banks 1/ (In percent)

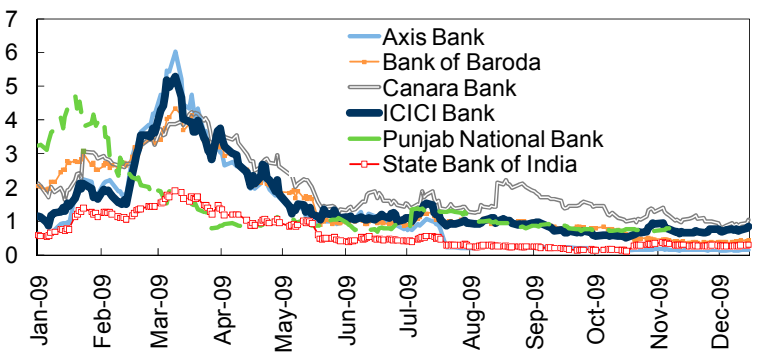

Source: Moody's KMV Creditedge Plus.

$1 /$ The Expected Default Frequency (EDF) is the probability that a company will default within a given time horizon, typically one year, where default means the failure to make a scheduled debt payment. 
Staff's stress tests indicate that if NPAs double relative to March 2009 levels, the average and median capitalization of the banking system remains above the minimum CAR of 9 percent. $^{1}$ However, sectoral weaknesses and concentration of corporate lending could result in a greater NPA increase: a 200 percent increase in NPAs would push a number of weaker public and smaller private banks below the minimum CAR. Changes discussed to the Basel capital regulations (i.e. the focus of unimpaired tier 1 capital and linkage to leverage) are not likely to require additional capital as Indian banks' share of hybrid capital and leverage ratios are moderate by international comparisons.

Nonbank institutions. After facing significant redemptions during the crisis, mutual funds have since witnessed large inflows especially from commercial banks. The inflows from banks have been to fixed maturity plans seeking yield and tax arbitrage opportunities given the high level of excess reserves, raising some concerns of liquidity risk if banks were to withdraw their deposits abruptly. NBFCs, which remain reliant on wholesale funding, are again expanding their assets, albeit at much reduced rates compared to the pre-crisis pace.

Staff broadly shares the authorities' assessment of India's financial stability, although more severe stress tests would be advisable. Credit remains the main risk to financial stability. The RBI's increase in provisioning coverage is welcome given India's low level. In line with the authorities' approach in previous cyclical upswings, higher provisioning on all real estate and equity exposures could also be considered, were signs of asset bubbles emerge. To deepen the financial stability assessment, more extreme stress tests for combined shocks should be carried out based on a more integrated approach to macro-financial risks: this could be incorporated in the guidelines on stress testing scheduled to be issued in January 2010.

The RBI's continued efforts to strengthen financial stability are welcome. The new Financial Stability Unity could help achieve a more integrated view of financial stability. The Financial Stability Report will provide more high frequency information on the financial sector, and could also fill information gaps regarding financial institutions' FX and derivatives exposures, and real estate indicators.

\footnotetext{
${ }^{1}$ The impact of higher default rates on CAR is estimated by assuming that impaired loans are provisioned up to 70 percent, and reducing risk-weighted assets and capital at a rate equivalent to the increase of the NPA ratio.
} 
Financial Market Developments

India's financial markets have rebounded.

Indian equity markets have outperformed most emerging markets..

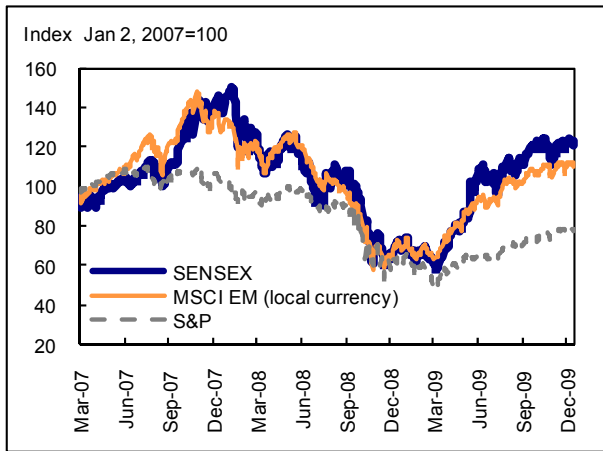

Sovereign credit conditions have normalized in line with declining global risk aversion...

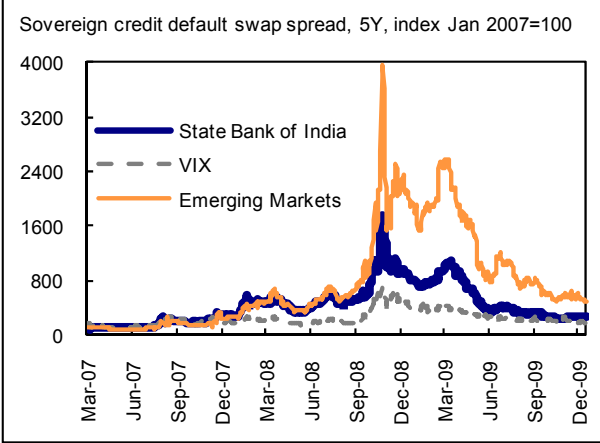

While government yields have risen, domestic credit yields have returned to pre-crisis levels.

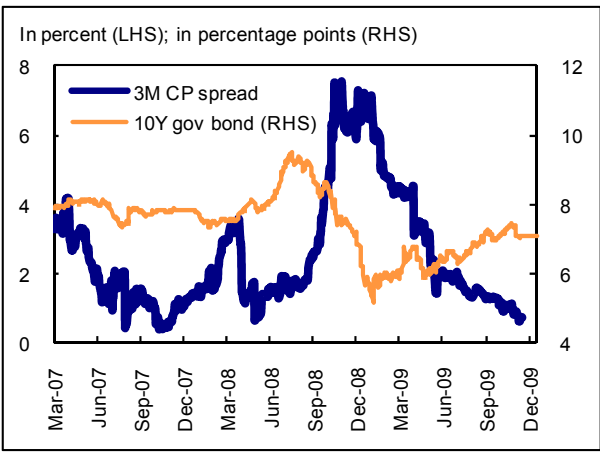

...led by a strong rebound in portfolio equity flows.

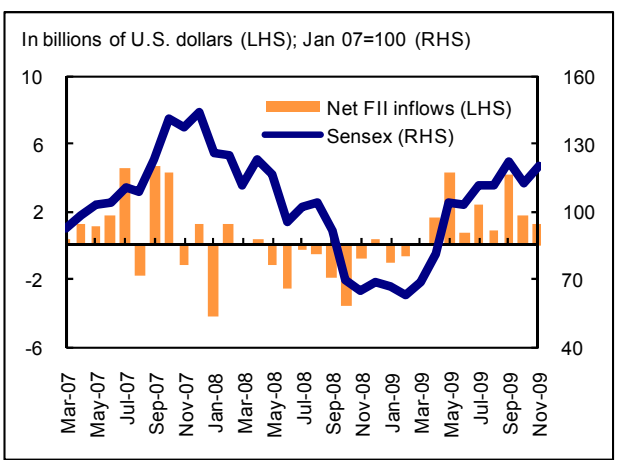

...resulting in lower external spreads for corporates.

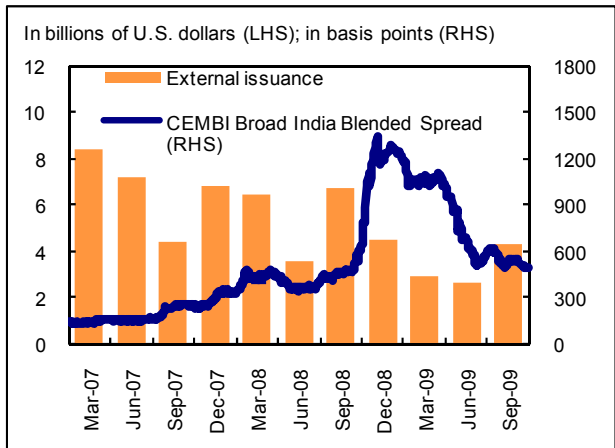

..spuring a flurry of domestic debt issuance.

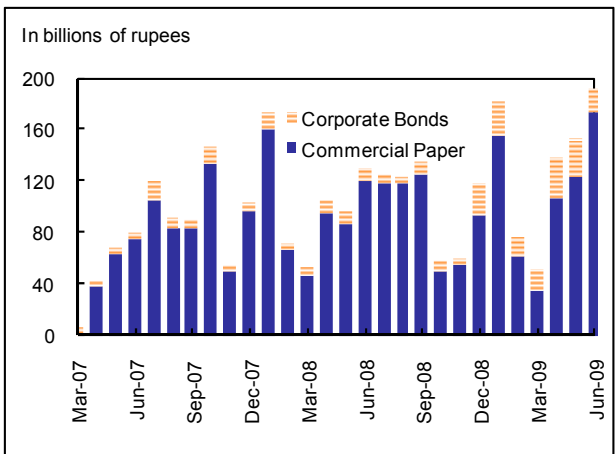

Sources: Reserve Bank of India; Bloomberg L.P.; Dealogic; and Fund staff calculations. 1/ Correlation between SENSEX and S\&P 500, monthly changes.

2/ AAA rated coporate bonds. 


\section{Appendix 8. Financial Sector Reforms: A Focus on Infrastructure Financing}

Reforms to the institutional framework for infrastructure financing will be critical to the achievement of the authorities' growth targets over the medium to long term. According to the Eleventh Five Year Plan (2007-12), sustaining a growth rate of 9 percent requires a substantial increase in infrastructure investment from around 5 percent of GDP to 9 percent of GDP by 2012. In turn, this calls for greater intermediation of India's savings, which, at 35 percent of GDP, are relatively high but partly locked up in physical assets (GOI 2007). ${ }^{1}$ At the same time, India's high debt and fiscal deficit absorb significant financial resources and preclude large-scale public-financed infrastructure projects, necessitating a greater reliance on private and public-private partnerships (World Bank 2006).

Increasing the size of the financial system and its capability to intermediate financial savings into infrastructure investment will be

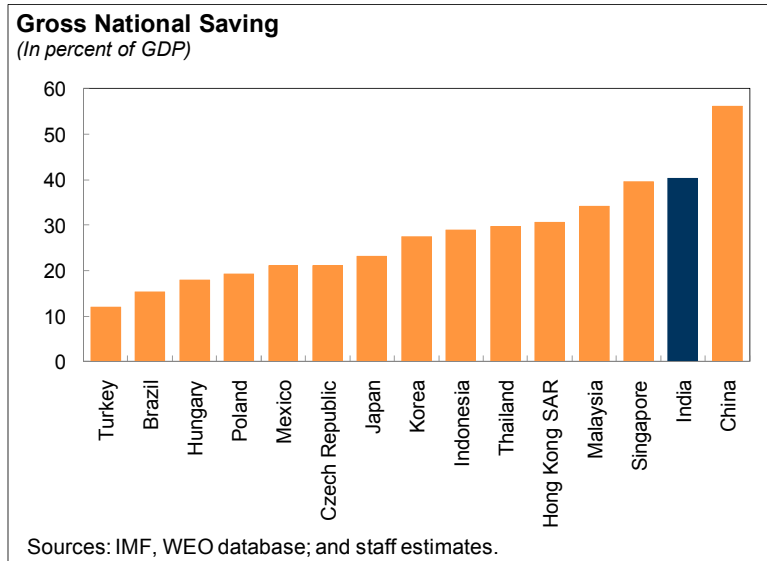
critical. Infrastructure financing has specific requirements such as the provision of long-term funds, varied risk-return characteristics, and a large and lumpy disbursement profile. India's financial system, which remains dominated by banks, is not particularly well suited for this kind of financing, while debt markets better able to finance infrastructure-especially the corporate bond marketremain relatively underdeveloped. Attempts to increase bank, pension, and insurance penetration will help mobilize long-term savings in the long run, but access to external finance-both in the form of foreign equity capital and long-term debt finance-is likely to be necessary in the short run (GOI 2007).

\section{Improving the Ability of Banks to Finance Infrastructure}

Banks have limited ability to finance infrastructure. A significant share of banks' assets, roughly corresponding to the size of their long-term liabilities, is reportedly already invested in infrastructure. Thus, banks would face considerable asset-liability mismatch if they were to expand their exposure to the sector much further. While the introduction of a takeout financing scheme by the India Infrastructure Financing Corporation (IIFCL)

\begin{tabular}{|c|c|c|c|c|}
\hline \multicolumn{5}{|c|}{$\begin{array}{l}\text { India: Bank Group-wise Maturity Profile of Select Liabilities/Assets } \\
\text { (As of end-March 2009; in percent of total) }\end{array}$} \\
\hline Assets/Liabilities & Public Sector Banks & Old Private Sector Banks & New Private Sector Banks & Foreign Banks \\
\hline \multicolumn{5}{|l|}{ I. Deposits } \\
\hline Up to 1 year & 45.7 & 48.3 & 53.1 & 63.8 \\
\hline Over 1 year and up to 5 years & 35.7 & 46.8 & 39.3 & 32.7 \\
\hline Over 5 years & 18.7 & 4.9 & 7.6 & 3.5 \\
\hline \multicolumn{5}{|l|}{ II. Borrowings } \\
\hline Up to 1 year & 70.8 & 76.7 & 44.3 & 84.8 \\
\hline Over 1 year and up to 5 years & 27.5 & 13.5 & 50.1 & 15.2 \\
\hline Over 5 years & 1.7 & 9.8 & 5.5 & 0 \\
\hline \multicolumn{5}{|l|}{ III. Loans and Advances } \\
\hline Up to 1 year & 39.1 & 40.8 & 32.4 & 55.8 \\
\hline Over 1 year and up to 5 years & 43.4 & 47.8 & 49.5 & 34.2 \\
\hline Over 5 years & 17.5 & 11.4 & 18.1 & 10 \\
\hline \multicolumn{5}{|l|}{ IV. Investments } \\
\hline a) Up to 1 year & 22.8 & 37.2 & 46.3 & 69 \\
\hline Over 1 year and up to 5 years & 30.4 & 18.2 & 30.5 & 24.8 \\
\hline d) Over 5 years & 46.8 & 44.7 & 23.2 & 6.2 \\
\hline
\end{tabular}
and the RBl's reduction in capital requirements for this type of financing could help banks alleviate such assetliability mismatch (Subbarao 2009), the availability of takeout financing in the country is very limited. Further, end-use restrictions on external commercial borrowing (ECB)-which prohibit post-

\footnotetext{
${ }^{1}$ Currently, about a third of gross domestic savings are in physical assets according to CSO statistics.
} 
implementation refinancing by foreign investors-also hold back the development of the takeout financing market. At the same time, a sufficiently developed foreign exchange (forward and derivative) market would also be needed for long-term foreign borrowing to be hedged. The successful introduction of exchange rate futures is welcome in this regard.

Figure 1. Selected Indicators of Source of Financing in Emerging Markets (2009)
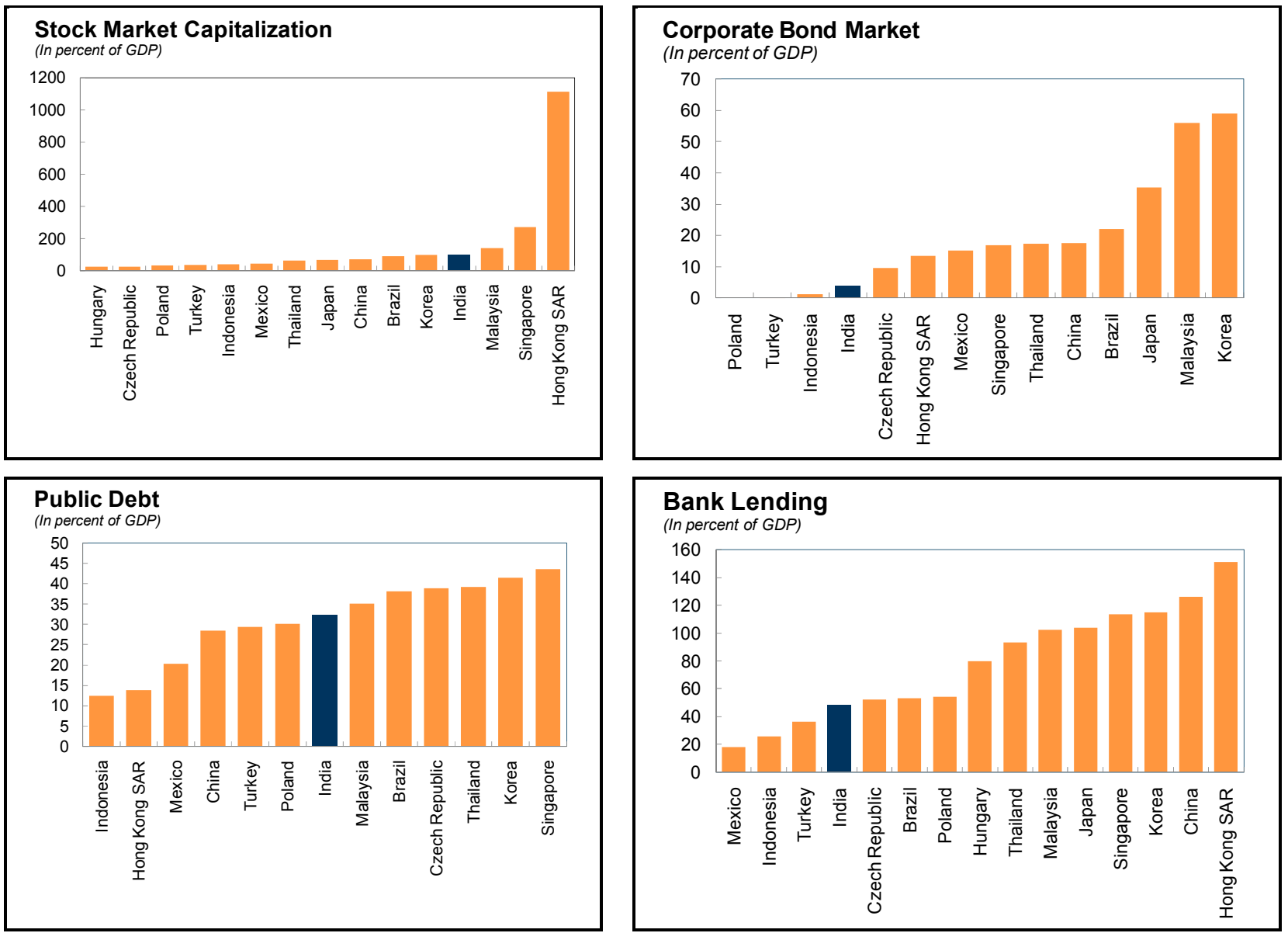

Source: Bank for International Settlements; Bloomberg Ltd; IMF International Financial Statistics; and IMF World Economic Outlook.

\section{Development of the Corporate Bond Market}

Further development of the corporate bond market is crucial to increase the availability of financing for infrastructure. ${ }^{2}$ India's corporate bond market remains small compared to other EMs, despite an increase in issuance in recent months and measures to promote its development. ${ }^{3}$ To

\footnotetext{
${ }^{2}$ Raising adequate equity finance tends to be one of the most challenging aspects of infrastructure project financing but India's relatively sophisticated and deep stock market makes it less of a binding constraint. Thus, we focus more on the debt capital angle of infrastructure financing. See World Bank (2006) for constraints on the equity-side.

${ }^{3}$ The authorities have streamlined primary issuance guidelines and disclosure and listing requirements for private placements, improved credit information, and strengthened clearing and settlement mechanisms.
} 
ensure that the recent burst in issuance does not prove ephemeral, long-term impedimentsincluding a lack of liquidity in the government bond market and the absence of a benchmark yield curve, limited third-party credit enhancement and hedging tools for investors and traders to mitigate credit risk and interest rate risk, poor and lengthy enforcement laws relating to default proceedings, and limited participation by domestic institutional investors-should be removed. ${ }^{4}$ In particular, the following issues need to be addressed:

- $\quad$ Government securities (G-Secs). A liquid G-Sec market is necessary to develop a benchmark yield curve for the pricing of corporate bonds. Despite the sizeable G-Sec market in India, liquidity remains low due to the lack of benchmark issues and the prevalence of buyand-hold investors. With regards to the former, the size of each benchmark security needs to be sufficiently large (usually a significant multiple of the average transaction size) in order to create a reliable benchmark yield curve. ${ }^{5}$ With regards to the latter, banks face a 25 percent statutory liquid ratio (SLR) held mostly in G-Secs, while institutional investors such as pension funds and insurance companies are mandated to hold a large share of their assets in G-Secs until maturity, thus hindering active trading. In addition, foreign institutional investors (FIls) - who are more likely to actively trade and thus provide liquidity - are only allowed to hold a very small

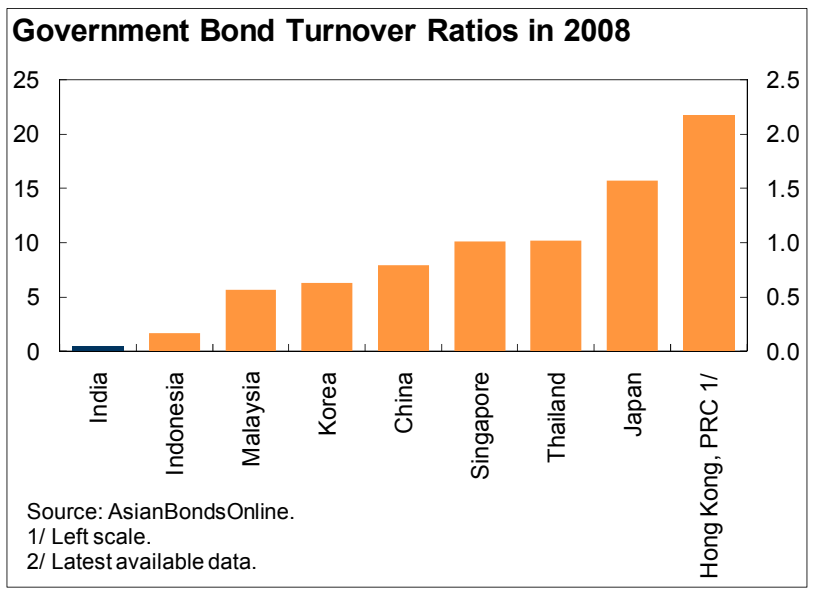
share of the outstanding stock of G-Secs (see below). Finally, the artificially high returns on alternative instruments such as the postal and small-scale saving schemes limit the attractiveness of G-Secs for retail investors.

- $\quad$ Credit enhancements. The issuance and trading of corporate bonds in India is dominated by bonds with a rating of $A A$ or above with little or no appetite for bonds rated at $A$ or below where most private infrastructure projects would fall. Third party credit enhancements or the provision of credit guarantees (also known as bond insurance) for issues could provide an impetus for lower rated issues to tap the market. While consideration is being given to the IIFCL providing such instruments, given the substantial infrastructure needs, it is unlikely that one institution would be able to meet the country's requirements. ${ }^{6}$ Other specialized public institutions might consider offering similar financial products, given that provision by private institutions is likely to be very limited and commercial banks are not permitted.

- $\quad$ Credit and liquidity risks. The RBl's proposal to introduce onshore single-name Credit Default Swaps (CDS) should improve investors' ability to trade credit risk. For this, however, a minimum level of market liquidity is necessary and therefore participation of Flls should be

\footnotetext{
${ }^{4}$ See GOI (2005), (2007a) and (2008) for recommendations to develop the corporate bond market in India.

${ }^{5}$ Starting in 2003, the RBI has made efforts to consolidate issuances with the aim of concentrating liquidity in a small number of benchmark issues but trading activity remains limited a few bonds and benchmark bonds often change suddenly.

${ }^{6}$ IIFCL might need to be adequately capitalized by the government or allowed to issue a quasi-sovereign international bond to fund a large volume of credit enhancements and would need to have a sufficient capital base to receive a AAA domestic rating in order to fulfill its role as a bond insurer.
} 
allowed. The authorities have also put forward draft guidelines on repos in corporate bonds that may enhance liquidity of the underlying asset. However, they have suggested that they would allow this only once fair pricing is available, although the two may be quite interconnected.

- Interest rate risks. The authorities re-launched interest rate futures (IRFs) on a 10-year Gsec in August 2009 following a failed attempt in 2001. The current guidelines allow authorized institutions to trade IRFs for both hedging purposes and on their own account which should enhance liquidity. ${ }^{7}$ However, the incentives to hedge interest rate risk remain limited by banks' ability to hold government securities up to the SLR limit in the banking book without applying mark-to-market accounting rules, while the limited liquidity of the underlying G-Secs hinders trading activity in IRFs. ${ }^{8}$

- $\quad$ Securitization. The RBIs proposed lock-in and minimum retention criteria for securitization exposures should help resolve concerns over the "Originate to Distribute" model viewed by many to be a contributor to the problems that arose in other structured finance markets during the global crisis. However, the retention requirements need not be imposed uniformly across the board, but tailored to the type of securitization and underlying assets to ensure that those forms of securitization that already benefit from "skin in the game" and operate well are not weakened (IMF 2009). The effects of other regulations such as revisions to the Basel II framework will also require careful consideration.

- $\quad$ Reducing costs of dealing with distressed assets. The market for distressed assets could be boosted by: (i) giving NBFCs the same rights as banks under the Securitization and Reconstruction of Financial Assets and Enforcement of Security Interest Act; (ii) easing restrictions on a single investor having a controlling stake in asset reconstruction companies (ARCs) and on FDI in ARCs and; (iii) creating a central registry of security interests. Passage of the new Companies Bill would consolidate the fora for dealing with the rehabilitation and liquidation of companies and bring the insolvency framework closer to international best practices. Further improvements-including mechanisms to deal with cross-border insolvency, a greater role to unsecured creditors, and allowing for debtor-in-possession financing - could be considered. In addition, ways to make the out of court corporate debt restructuring mechanism result in more operational restructuring could be explored and the personal insolvency framework modernized.

- Development of the corporate bond market would benefit from increasing the role of foreign investors in the local debt market. Domestic institutional investors are typically buy-and-hold investors, while foreign participation are more likely to trade and could therefore contribute to a more liquid market. Although the authorities have increased the limit on foreign participation in the corporate bond market from $\$ 3$ to $\$ 15$ billion, these limits have not been fully utilized. The reasons mentioned by investors include the fact that the market is too small and illiquid, the lack of a benchmark yield curve in the government bond market as referred to above, as well as a lack of transparency surrounding the allocation mechanism for FII limits. For this reason, the authorities could consider merging the existing limits with those for the government bond market (currently $\$ 5$ billion). The authorities remain concerned that this will increase volatility in the market. However, a staff study of 10 major EMs revealed that while greater foreign participation lowers yields, the effect on the volatility of yields cannot be clearly discerned: greater foreign participation reduced volatility in Mexico, Malaysia, Turkey

\footnotetext{
${ }^{7}$ Trading in IRFs was only allowed on a client's account when IRFs were launched in 2001.

${ }^{8}$ There are also issues concerning the physical settlement of the IRF contract that may constrain its development.
} 
but increased it in Korea and no relationship between the two was detected in several other countries. ${ }^{9}$

\section{The Role of Institutional Investors}

International experience suggests that domestic institutional investors play a key role in the development of the corporate bond market. In several Latin American countries (e.g. Chile and Mexico), growth in the amount of corporate bonds outstanding was closely tied to the growth of pension fund assets under management (AUM). Insurance companies have also been a significant source of demand for corporate bonds in Asia-including in Malaysia where an easing of regulations on portfolio composition played an important role. Mutual funds have also become a more important player in the fixed income markets globally.

$\bullet$

In India, the New Pension System (NPS)-which was expanded to include unorganized sector workers in 2009-has enormous potential to mobilize long-term savings. ${ }^{10}$ In 2008 , the authorities set up a special trust, selected a custodian bank, held a competitive bidding process for asset managers, and introduced a central record keeping authority (CRA). This has increased the number of investment options for participants and should help raise the attractiveness of the scheme. Expansion of the NPS - together with the

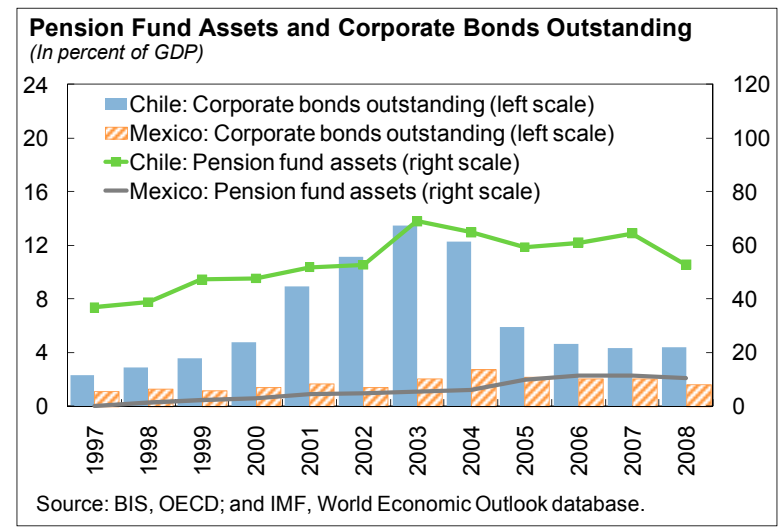
proposed Pension Fund Regulatory and Development Authority (PFRDA) bill—should lead to greater demand for corporate bonds as NPS investment options and guidelines are less restrictive than the Employees' Provident Fund Organization (EPFO) investment regulations. ${ }^{11}$ However, for the time being most of the AUM of pension and provident funds are under EPFO, which does not invest in corporate bonds. $^{12}$

\footnotetext{
${ }^{9}$ See Peiris (2009).

10 The NPS is still in its infancy and currently covers only about 600,000 government employees and 3,000 nonpublic sector workers with total NPS assets at about Rs 33 billion. See Poirson (2007) for an overview of pension reforms and its financial implications in India.
}

\footnotetext{
${ }^{11}$ The PFRDA Bill proposes that the main mandate of PFRDA is to regulate the NPS. Pension schemes already covered under the Employees' Provident Fund \& Miscellaneous Provisions Act, 1952 and other enactments would be specifically excluded from the architecture of the NPS. Further, the Bill provides that PFRDA will stipulate investment guidelines for pension funds.
}

${ }^{12}$ A survey of pension fund investment guidelines in other EMs suggests that while significant exposure to government debt is stipulated, there is considerable freedom for these funds to invest in a mix of financial instruments with varying risk-return profiles more akin to the investment option under the NPS. 
- Increasing the penetration of life insurance in India-which remains relatively low--would raise demand for corporate bonds. The proposed Insurance Bill amendment-which raises the foreign ownership limit in insurance companies from 26 to 49 percent-should help attract large foreign players into the market and the low level of insurance penetration suggests that insurance companies' AUM will increase significantly. ${ }^{13}$ However, investment guidelines would

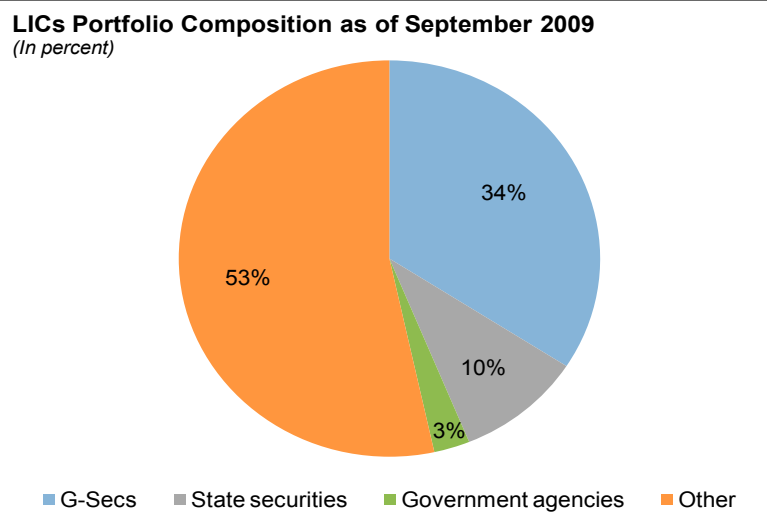
have to be relaxed ${ }^{14}$ and the insurance

G-Secs $\backsim$ State securities $\square$ Government agencies $\square$ Other companies' role as captive sources for government financing remains a key obstacle to their investments in other fixed-income products. The Life Insurance Corporation of India (LIC), which has over 70 percent of market share, holds 22 percent of the stock of government securities.

- The sharp increase in AUMs of fixed maturity plan mutual funds (MFs) in India could provide an important source of demand for corporate paper. Overall, there is a need for an analysis of scope for tax and regulatory arbitrage among the pension, insurance and MF sectors to support their sound growth given the nascent state of institutional development.

\footnotetext{
${ }^{13}$ Non-life insurance companies have shown a tremendous growth in recent years largely driven by unit linked insurance policies (ULIPs) that invest predominantly in equities rather than fixed-income instruments.

${ }^{14}$ The investment guidelines revised by the Insurance Regulatory and Development Authority (IRDA) in 2008 require insurance companies to invest not less than 15 percent of their investments in infrastructure and social sectors. However, the guidelines also lay down a minimum tenor of 10 years and a credit rating of 'AA' for investments in debt paper which would exclude most infrastructure SPVs' debt paper. Investment guidelines for life and non-life insurance companies also specify that not less than 50 percent of assets should be in government or approved securities, which effectively renders nearly 50 percent of assets to be invested in public sector debt due to restrictions on approved securities.
} 


\section{References}

Asian Development Bank. 2008. "Are There Ways to Broaden Investor Diversity?" Asia Bond Monitor, April, Manila

Government of India, Ministry of Finance, 2007a "Report of the High Level Expert Committee on Making Mumbai an International Financial Centre", New Delhi

_2007b, "The Report of the Committee on Infrastructure Financing" Planning Commission. New Delhi

2005, "Report of the High Level Expert Committee on Corporate Bonds and Securitization (the Patil Report)", New Delhi

2008, "Report of the Committee on Financial Sector Reforms", New Delhi

International Monetary Fund, (2005), "Development of Corporate Bond Markets in Emerging Market Countries", in the Global Financial Stability Report (September 2005)

2008, "Restarting Securitization Markets: Policy Proposals and Pitfalls" in the Global Financial Stability Report (October 2009)

McKinsey, 2009, "Rebuilding India - Financing and Investing in Infrastructure"

Mohan, R. (2006), "Recent Trends in the Indian Debt Market and Current Initiatives. Reserve Bank of India," Mumbai

Mohan, R. and Shri A. Chawla, Shri A. Jha, and D. Subbarao (2009), "India's Financial Sector - An Assessment," Committee on Financial Sector Assessment

Nair, T. C. 2007. "Development of the Corporate Bond Market in India: Tasks Ahead,"

Peiris, S. J., (2009), "Foreign Participation in Emerging Markets Local Currency Bond" IMF working paper (forthcoming).

Poirson, H., (2007), "Financial Market Implications of India's Pension Reform”, IMF Working Papers $07 / 85$

Securities and Exchange Board of India, 2008, "Developments in the Corporate Bonds and Securitization Markets-An Update," August. Mumbai

World Bank, 2009, "Financing Public Private Partnerships (PPPs) in Infrastructure through Support to the India Infrastructure Finance Company Limited" Project Appraisal Document

2006, "India Financing Infrastructure: Addressing Constraints and Challenges" 


\section{Appendix 9. Foreign Participation in Emerging Markets Local Currency Bond Markets ${ }^{1}$}

Foreign investors can be an important source of demand for domestic government paper, thus lowering bond yields and improving market liquidity. On one hand, foreign investors could help develop domestic bond markets in Emerging Markets (EMs) as their higher propensity to trade complements the buy-and-hold strategy of most domestic institutional investors, which contributes to a more liquid market. On the other hand, increased foreign presence could also imply greater volatility in local bond markets as highlighted by the literature on "sudden-stops" of capital flows and evidence of contagion in EMs.

This paper sheds light on the impact of foreign participation on long-term government bond yields and their volatility in a group of EMs. To our knowledge, there has been little empirical analysis of the determinants of local currency bond market yields in EMs, in general, and even less on the role of foreign investors. ${ }^{2}$ For this study, we compile a unique time-series database of foreign participation in domestic government bond markets from the Asiabondonline database and IMF country desk data.

\section{Panel data estimates indicate that foreign participation} reduces long-term local currency bond yields in EMs.

The econometric analysis is based on a standard reducedform specification for a panel of $10 \mathrm{EMs}$ using a comprehensive set of macro-financial determinants identified in the literature and country fixed effects. Importantly, the results suggest that foreign participation has a statistically significant impact on yields controlling for other determinants. A 1 percent increase in the share of foreign investors in the government bond market is associated with 6 bps lower yields, on average.

However, there is no clear evidence that foreign investors increase interest rate volatility. We use a time series approach to estimate the impact of the share of foreign investors in government bond markets on the volatility of long-term yields in EMs. Using a Generalized Autoregressive Conditional Heteroskedasticity (GARCH) econometric model for monthly data from 2000 , we show that greater foreign participation significantly increases yield volatility in one country (Korea) and decreases it in a few others (Hungary, Malaysia, Mexico, Turkey), while being an insignificant explanatory variable in most other cases.
Determinants of long-term domestic government bond yields

\begin{tabular}{|lr|}
\hline Policy interest rates & 0.12 \\
& $(4.63)$ \\
\hline Inflation & 0.17 \\
& $(5.26)$ \\
\hline Fiscal deficit & 0.05 \\
& $(1.74)$ \\
\hline Foreign participation & -0.06 \\
& $(4.82)$ \\
\hline Current account & 0.12 \\
& $(4.70)$ \\
\hline Constant & 5.38 \\
& $(15.82)$ \\
\hline R-Square (within) & 0.4 \\
\hline F-statistic & 129.3 \\
\hline
\end{tabular}

Note: Results are from a fixed effect specification on a panel of $10 \mathrm{EMs}$ for the period 2000Q1-2009Q1. The tstatistics are in parentheses.

\footnotetext{
${ }^{1}$ This is a summary of Peiris, S. "Foreign Participation in Emerging Markets Local Currency Bond Markets," IMF Working Paper, forthcoming.

2 The lack of data on foreign participation in EMs has hampered such a study to date: foreign participation is defined as the share of the outstanding stock of domestic government securities held by non-residents.
} 


\section{Appendix 10. Fiscal Issues}

\section{Background}

India's public finances continue to be under strain. The fiscal deficit has deteriorated significantly since $2008 / 09$, when a combination of spending measures introduced prior to the global crisis, a soaring subsidy bill, fiscal stimulus packages in response to the crisis, and a cyclical downturn in tax revenue, reversed the process of fiscal consolidation underway since $2003 / 04$. The 2008/09 general government fiscal deficit, inclusive of subsidy-related bond issuance, widened to 11 percent of GDP, one of the highest among EM, from 4.7 percent of GDP in 2007/08.

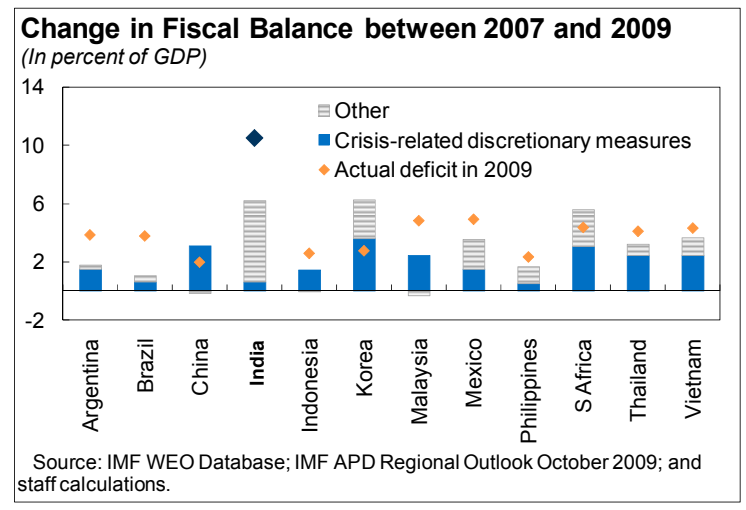

The general government deficit will likely remain unchanged at about 11 percent of GDP in 2009/10

- Central government. The 2009/10 budget, with a deficit target of 6.8 percent of GDP, extends the expansionary fiscal stance to help support economic recovery. The high level of public spending is maintained with savings from the lower subsidy bill reoriented towards social programs and infrastructure. Tax revenue is expected to remain subdued reflecting the 6 percentage points cut in excise and 2 percentage points cut in service tax rates. Staff estimates that the central government's deficit target for this year may exceed the budget target marginally (by 0.2 percent of GDP), as indirect tax revenue may underperform given collections so far. The robust economic recovery underway may boost direct tax collections and close the gap on the revenue side, but spending pressures constitute the main downside risk. Subsidy-related bond issuance is projected at 0.3 percent of GDP.

- $\quad$ States. The combined states' deficit is projected to widen to 3.7 percent of GDP in 2009/10, from an estimate of 3.1 percent of GDP in 2008/09. States are implementing the recommendations of the $6^{\text {th }}$ Pay Commission and are taking advantage of the higher borrowing limit to raise capital spending, while growth in own revenue and resources transferred from the center remains lackluster.

The targeted reduction in the central government's budget deficit in the next two years is feasible, but requires measures. The goal to reduce the deficit to 5.5 percent of GDP in 2010/11 from a projected 7 percent in the current year will be helped by: (i) the falling out of some expenditure-the Sixth Pay Commission arrears and lower outlays for the agricultural loan waiver (about 0.5 percent of GDP); and (ii) revenue buoyancy ( 0.4 percent of GDP). But some additional discretionary measures, such as the rolling back of the tax cuts implemented during the crisis, are likely to be needed. The reduction in the deficit to 4 percent of GDP announced for 2011/12 will unlikely be achieved without expenditure measures and the implementation of the planned tax reforms.

International spillovers of India's fiscal consolidation are likely small. In India's case, lowering the deficit could actually be expansionary. Further, the international impact may be positive because investment is more import-intensive than government consumption. 


\section{Medium-term fiscal reforms}

\section{Revenue reform has the potential to} contribute to fiscal consolidation over the medium-term. Two major tax reforms are on the anvil that aim to broaden the tax base, while lowering rates. As India's tax revenues are high relative to peers, both reforms will be designed to be revenue neutral. Nevertheless, they are anticipated to yield substantial efficiency gains and improve compliance which would bolster economic performance. ${ }^{1}$

- $\quad$ The authorities have issued a draft Direct Tax Code, which would bring simplicity and transparency, and minimize distortions. The draft Direct Tax Code broadens the tax base by removing most exemptions, and proposes reducing corporate tax rates from 33 to 25 percent. $^{2}$ The Direct Tax Code, which is currently being revised to incorporate comments from stakeholders, is expected to be adopted in 2011/12.

-

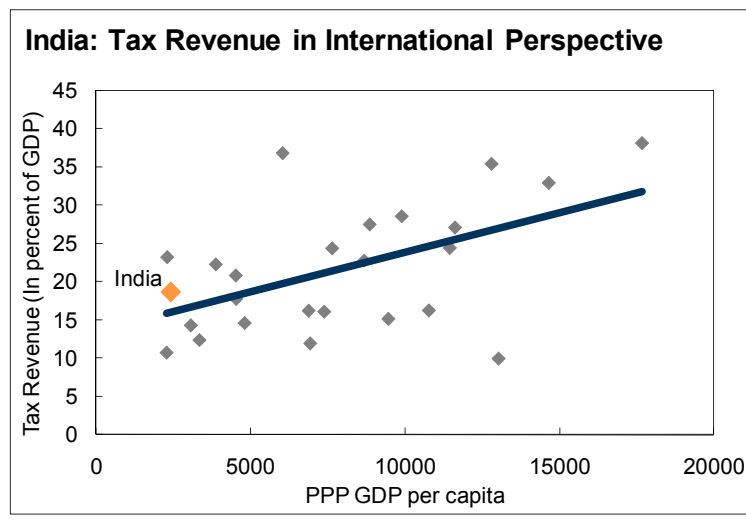

The authorities are also planning to introduce a Goods and Services Tax (GST), whose exact parameters are currently being decided. The GST will have a federal and state component and will subsume various indirect central and state taxes. By reducing the cascading of indirect taxes, harmonizing tax rates and exemptions across states, and establishing a comprehensive and transparent chain of set-offs, the GST will widen the tax base and improve compliance, eventually leading to lower tax burden, which would in turn feed into higher direct tax collections. ${ }^{3}$

The missing element to the authorities' plans is expenditure reform. To ensure a lasting fiscal consolidation, subsidy reform remains the topmost priority. ${ }^{4}$ The reform of the subsidy system should eliminate regressive subsidies (such as on gas, diesel and LPG) and introduce a market-based pricing mechanism for petroleum products. Over time, the subsidies on products consumed predominantly by the poor, e.g. kerosene, food, fertilizer, could be replaced by targeted support, which should be facilitated by the planned introduction of a unique identification number. These measures would go a long way in lowering the subsidy bill without unduly affecting the poor. ${ }^{5}$

\footnotetext{
${ }^{1}$ The introduction of a 'flawless' GST is estimated to boost GDP growth by 0.9-1.7 percent (Report of the Taskforce on Goods \& Services Tax, $13^{\text {th }}$ Finance Commission, December 15, 2009).

2 In 2008/09, revenue foregone by the central government from existing exemptions amounted to 8 percent of GDP and close to 70 percent of actual tax collection.
}

${ }^{3}$ If successful, the reform will not only significantly improve the country's tax system but will also pave the way forward for other countries by resolving a longstanding dilemma for VAT administrators-how to best implement a multijurisdictional VAT system.

\footnotetext{
${ }^{4}$ See Selected Issues Paper, Chapter 7, India 2007 Article IV Consultation and Selected Issues Paper, Chapter 1, India 2005 Article IV Consultation.

5 The Central Government Subsidies Report (2004) estimates that eliminating non-essential subsidies, and better targeting food, oil and fertilizer subsidies could save up to 2.3 percent of GDP.
} 
Continued monitoring and evaluation of centrally sponsored schemes and rationalization, when needed, should enhance the quality of public spending and improve service delivery, critical for sustaining inclusive economic growth and alleviating poverty.

\begin{abstract}
A faster pace of privatization of public sector companies could also support fiscal consolidation, but cannot replace adjustment. The government recent announcement that all listed public sector undertakings (PSU) have to meet a minimum 10 percent public holding requirement and all profitable PSU must be listed is a step in the right direction. But for a significant contribution to debt reduction, the government would have to divest some of the companies it now judges strategic and lower the minimum government holdings in the financial sector.
\end{abstract}


India: Fiscal Indicators

\section{India's fiscal position remains weak.}

India's government finances continue to be under strain as the 2009/10 budget...

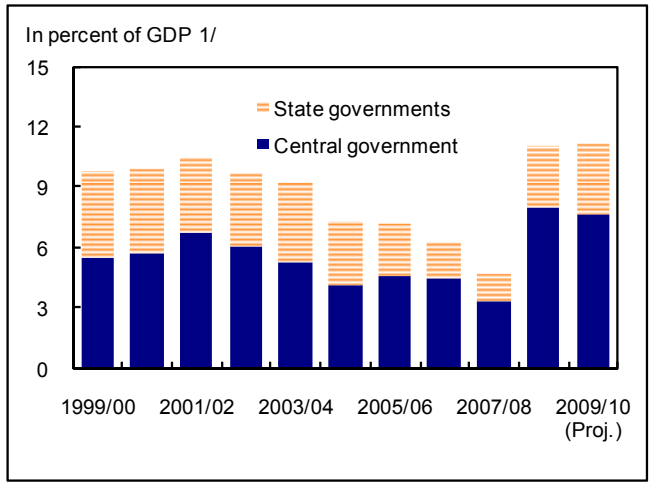

...while the economic slowdown and indirect tax cuts dragged revenues.

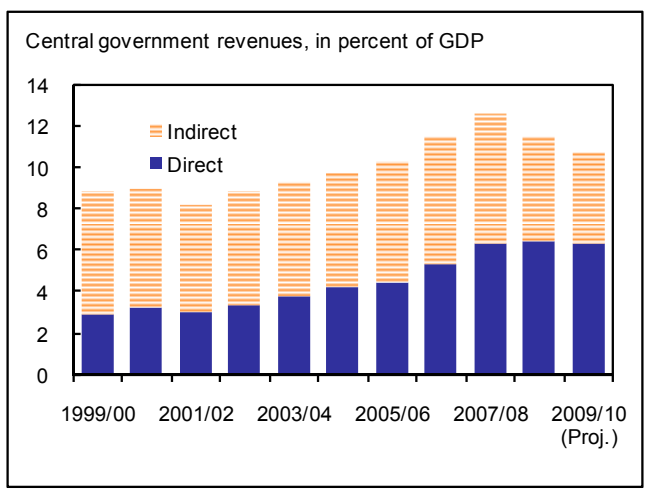

Returning to pre-crisis levels of fiscal deficits will likely be a long road ahead...

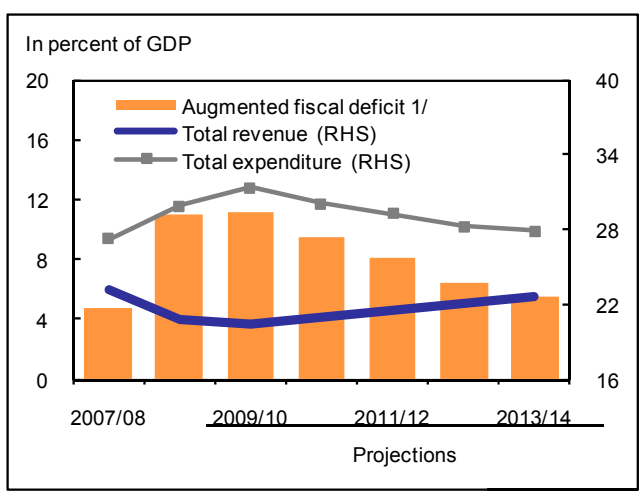

... maintained the expansionary fiscal stance with high levels of spending...

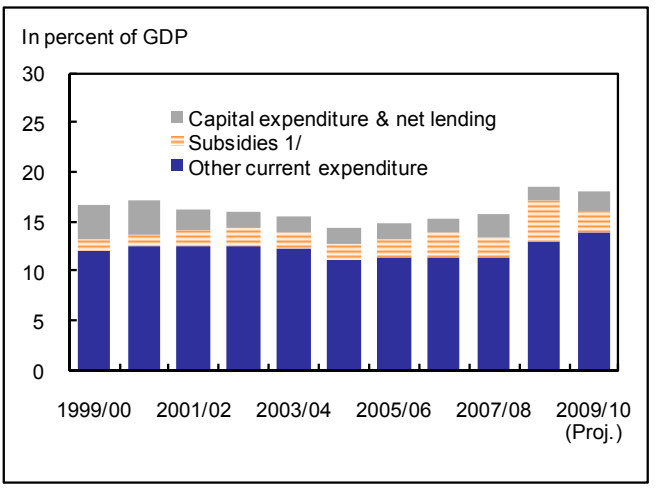

As a result, public debt is on the rise again.

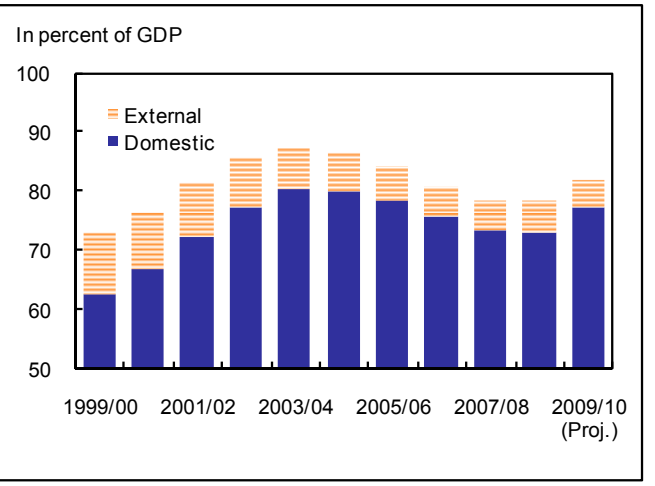

....and public debt is projected to decline marginally in the absence of measures.

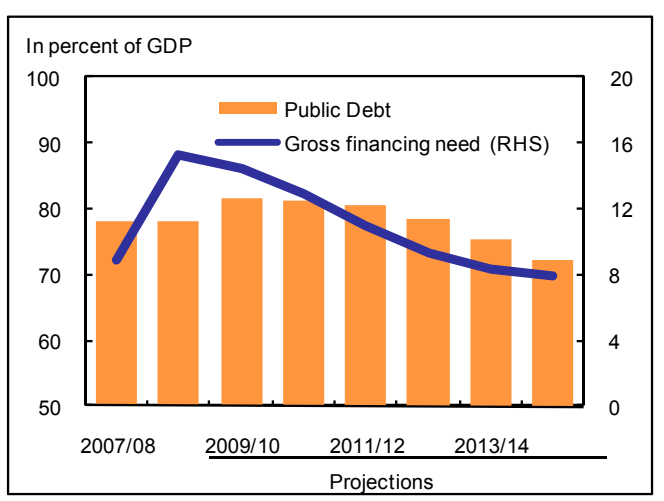

Sources: Country authorities; and Fund staff calculations.

$1 /$ Includes subsidy-related bond issuance. 


\section{Appendix 11. Public Debt Target ${ }^{1}$}

Determining the appropriate level for a public debt target is challenging. With economic theory providing little guidance on the optimal level of debt, various approaches were used to examine what would be a prudent and feasible debt ratio for India.

Cross-country studies have identified a wide range of 'optimal' levels of public debt and most countries have opted for simple rules of thumb in selecting their target. Depending on countries' circumstances and policy priorities, debt targets range from as low as 25 percent to as high as 60-70 percent of GDP. Most countries appear to have followed convergence criteria of regional economic agreements and peers' debt ratios. Debt ceilings cluster around 60 percent and 40 percent of GDP.

Relative to EMs, India's public debt is substantially higher and the gap has widened over time. India's public debt at 78 percent of GDP in $2008 / 09$ stands out against the average for EM at 45 percent of GDP. In 2003, these ratios were 87 percent and 62 percent of GDP, respectively.

Following Reinhart and others (2003), ${ }^{2}$ the public debt thresholds of debt intolerance for India range from $\mathbf{4 0}$ to $\mathbf{7 5}$ percent of GDP. This methodology relates a country's sovereign rating (proxied by the Institutional Investor Rating) and its public debt-to-GDP ratio, controlling for inflation and default history, to determine the level of debt at which the country will be deemed more credit-worthy. In the full sample of countries, India's threshold for debt intolerance is estimated to be as high as 75 percent. Once the sample is restricted to countries with market access, the threshold declines to 40-45 percent of GDP.

The choice of a debt target should also be informed by intertemporal solvency considerations, as captured in the present discounted value of expected future primary surpluses. ${ }^{3}$ Persistent primary deficits and negative projected

\begin{tabular}{|c|c|}
\hline \multicolumn{2}{|c|}{ Examples of Debt Ceilings } \\
\hline 30 & Namibia \\
\hline 40 & $\begin{array}{c}\text { Mercosur, UK, } \\
\text { Denmark, Ecuador, } \\
\text { Panama, Kenya }\end{array}$ \\
\hline 50 & $\begin{array}{c}\text { Andean } \\
\text { Community, Central } \\
\text { American Countries }\end{array}$ \\
\hline 60 & $\begin{array}{c}\text { EU, Estonia, } \\
\text { Indonesia, } \\
\text { Pakistan, Poland }\end{array}$ \\
\hline 70 & CEMAC, WAEMU \\
\hline
\end{tabular}

Real Growth and Reduction of Public Debt 2003-2008 (In percent)

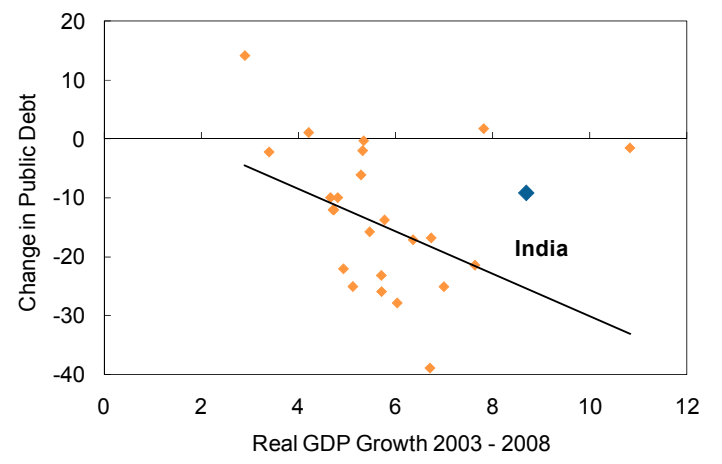

\footnotetext{
${ }^{1}$ Nyberg, D. and P. Topalova (2009). "What Level of Public Debt Could India Target," IMF Working Paper No. $10 / 7$.

${ }^{2}$ Reinhart C., K. Rogoff, and M. Savastano (2003). "Debt Intolerance," Brookings Papers on Economic Activity, $2003(1)$

${ }^{3}$ Abiad, A. and J. Ostry (2005). "Primary Surpluses and Sustainable Debt Levels in Emerging Market Countries," IMF Policy Discussion Paper 05/06.
} 
interest growth differentials preclude the use of this methodology in India's case. Nevertheless, sensitivity analysis reveals that a 60 percent debt-to-GDP ratio can be sustained under reasonable assumptions for India's long-run primary balance and discount factor.

India's public debt could follow an array of paths depending on the fiscal reforms that the government implements. By 2015/16, public debt could decline only marginally under a no-reform scenario, while a combination of a fuel subsidy reform, enhanced revenue performance, and partial privatization of public enterprises could bring its level to about 62 percent of GDP.

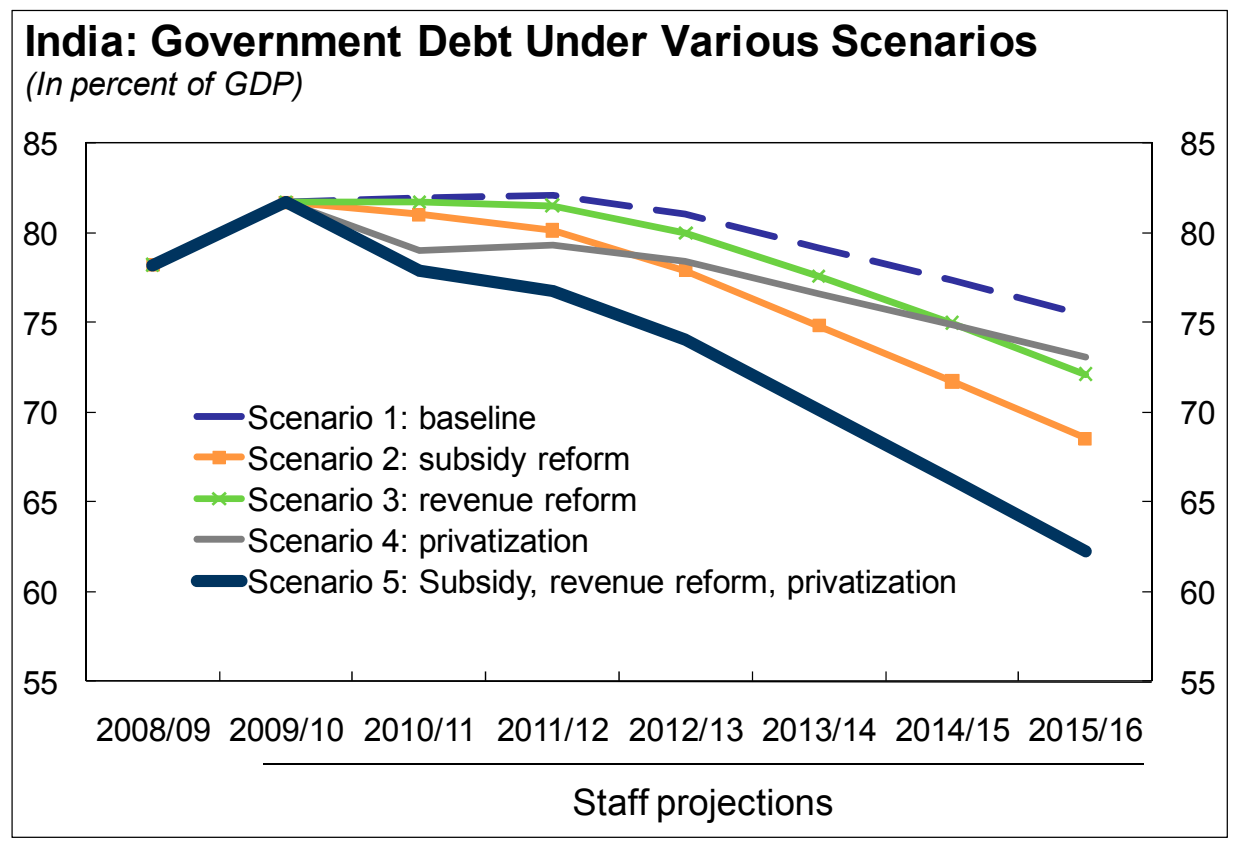

While none of the approaches yields conclusive results individually, taken together, they suggest that a reasonable and feasible public debt ceiling for India by 2015/16 could be in the order of 60-65 percent of GDP. Such a debt ceiling, while still above the average for EMs, would allow for a countercyclical fiscal policy and provide some headroom for large contingent liabilities. It is also a level of debt that could be sustainable given reasonable assumptions about the future interestgrowth differentials and primary balances. This debt target, lower than India's lowest debt ratio over 20 years, would also be a clear break from the past and would, thus, bolster the credibility of India's fiscal framework. 


\section{INTERNATIONAL MONETARY FUND}

INDIA

\section{Staff Report for the 2009 Article IV Consultation-Informational Annex}

Prepared by the Asia and Pacific Department

January 11,2010

Contents

Page

I. Medium-Term Public Debt Sustainability Analysis ............................................... $\underline{2}$

II. Medium-Term External Debt Sustainability Analysis............................................. $\underline{5}$

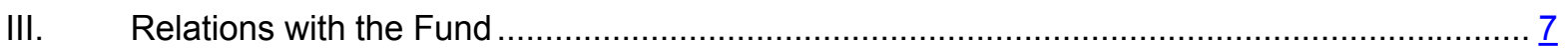

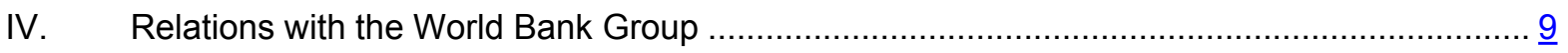

V. Relations with the Asian Development Bank ............................................................. 11

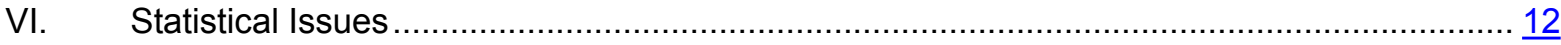




\section{Annex I: India—Medium-Term Public Debt Sustainability Analysis}

Staff assumptions. After a dip in 2009/10, economic growth is forecast to quickly recover to its potential (8 percent) driven by strong domestic investment and private consumption. Inflation is projected to gradually decline to 5-6 percent by end-2010/11 and remain contained thereafter. The general government deficit is projected to steadily decline, as the effect of the Sixth Pay Commission wage hike, agricultural relief and fiscal stimulus wears off. Even without assuming a roll back of the indirect tax cuts during the crisis, tax revenue is expected to recover gradually to its pre-crisis level as a share of GDP, as the implementation of the goods and services tax in 2011/12 raises the buoyancy of tax collections. Other working assumptions include:

- $\quad$ Oil bonds. After 2009/10, the government is assumed to issue oil bonds to the state-owned petroleum companies only to cover the subsidy cost of kerosene.

- $\quad$ Other subsidy-related bond issuance. After 2009/10, the government is assumed not to issue further bonds to the Food Corporation of India or the fertilizer companies as these expenses are brought on-budget. Given the projected improvements in targeting and the move towards nutrient-based fertilizer regime, the non-oil subsidy bill is assumed to decline marginally as a share of GDP.

Debt path. Under the baseline scenario, the general government deficit would fall to 5.1 percent of GDP by 2014/15 from roughly 11 percent of GDP in 2009/10. However, even at the end of the period, the current balance of the general government continues to be in deficit. Over the same period, gross public debt would decline to 72.3 percent of GDP from 78.2 percent of GDP in 2008/09, although it is projected to peak at 81.7 percent of GDP reflecting the double-digit deficit of 2009/10.

\section{A. Sensitivity Analysis}

After an initial rise, a gradual decline in the debt-to-GDP ratio would occur under various shocks, including in a scenario where the growth-interest rate differential rises above its historical average or the currency experiences a sharp depreciation (Figure I.1 and Table I.1).

However, these favorable debt dynamics are contingent on a concerted effort by the government to reduce the fiscal deficit. A no-policy change scenario, in which the primary deficit is held equal to its 2009/10 value, would lead to an unsustainable debt path. The debt-to-GDP dynamics are also highly sensitive to the growth assumptions. In the case of a protracted slowdown, with real GDP growth averaging 5 percent, the ratio of debt to GDP would rise over the medium term. 
Figure I.1. India: Public Debt Sustainability: Bound Tests 1/

(Public debt, in percent of GDP)

Baseline and historical scenarios

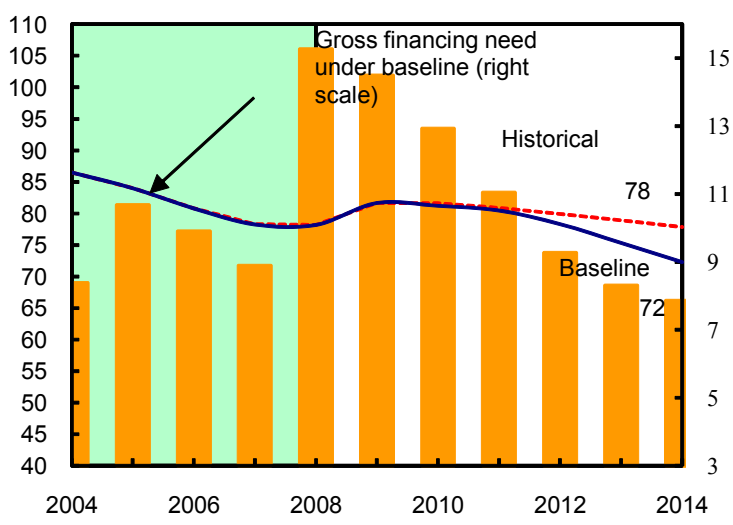

Growth shock (in percent per year)

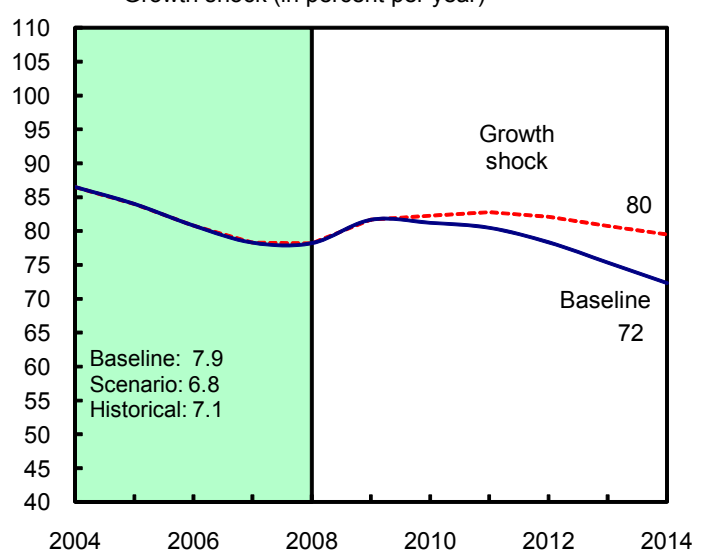

Combined shock 2/

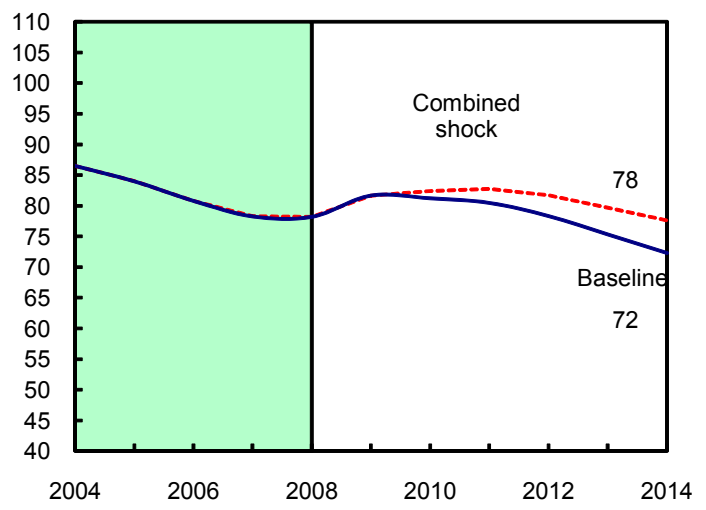

Interest rate shock (in percent)

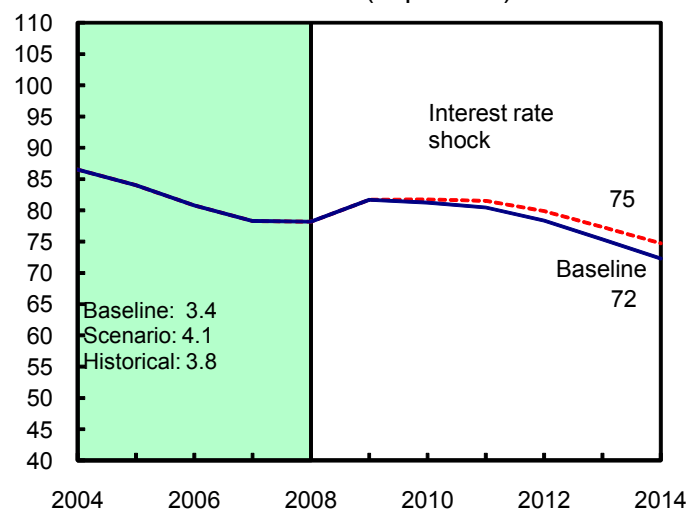

Primary balance shock (in percent of GDP) and no policy change scenario (constant primary balance)

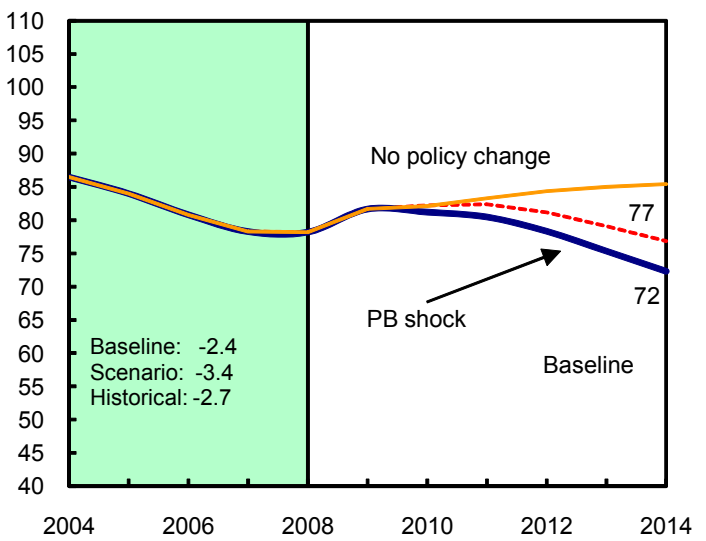

Real depreciation and contingent liabilities shocks 3/

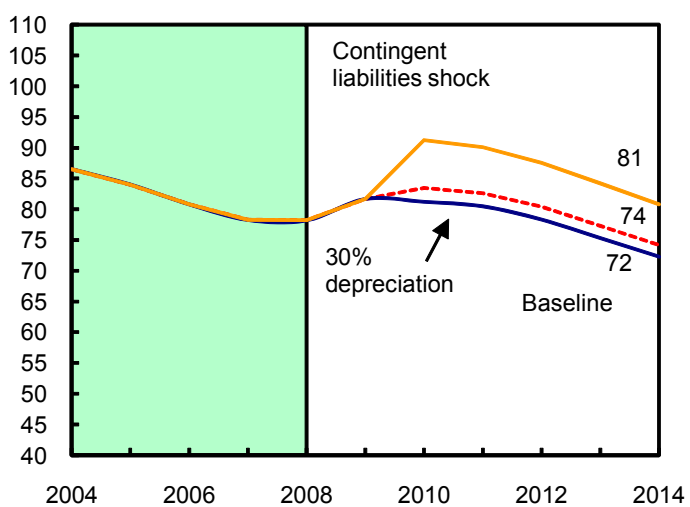

Source: Fund staff estimates; fiscal year data.

$1 /$ Shaded areas represent actual data. Individual shocks are permanent one-half standard deviation shocks. Figures in the boxes represent average projections for the respective variables in the baseline and scenario being presented. Ten-year historical average for the variable is also shown.

2/ Permanent 1/4 standard deviation shocks applied to real interest rate, growth rate, and primary balance.

3 / One-time real depreciation of 30 percent and 10 percent of GDP shock to contingent liabilities occur in 2009, with real depreciation defined as nominal depreciation (measured by percentage fall in dollar value of local currency) minus domestic inflation (based on GDP deflator). 
Table I.1. India: Public Sector Debt Sustainability Framework, 2004/05-2014/15

(In percent of GDP, unless otherwise indicated)

\begin{tabular}{|c|c|c|c|c|c|c|c|c|c|c|c|}
\hline \multirow[b]{3}{*}{$\begin{array}{l}\text { Baseline: Public sector debt } 1 / \\
\text { o/w foreign-currency denominated }\end{array}$} & \multicolumn{5}{|c|}{ Actual } & \multicolumn{6}{|c|}{ Projections } \\
\hline & \multirow{2}{*}{$\begin{array}{r}2004 / 05 \\
86.5\end{array}$} & $2005 / 06$ & $\begin{array}{r}2006 / 07 \\
80.8\end{array}$ & $\begin{array}{r}2007 / 08 \\
78.3\end{array}$ & $\begin{array}{r}2008 / 09 \\
78.2\end{array}$ & $\begin{array}{r}2009 / 10 \\
81.7\end{array}$ & $\begin{array}{r}2010 / 11 \\
81.2\end{array}$ & $\begin{array}{r}2011 / 12 \\
80.5\end{array}$ & $\begin{array}{r}2012 / 13 \\
78.3\end{array}$ & \multirow{2}{*}{$\begin{array}{r}2013 / 14 \\
\\
\end{array}$} & \multirow{2}{*}{$\begin{array}{r}2014 / 15 \\
\\
72.3 \\
4.1\end{array}$} \\
\hline & & $\begin{array}{r}84.0 \\
5.7\end{array}$ & $\begin{array}{r}80.8 \\
5.0\end{array}$ & $\begin{array}{r}78.3 \\
4.7\end{array}$ & $\begin{array}{r}78.2 \\
5.0\end{array}$ & $\begin{array}{r}81.7 \\
4.6\end{array}$ & $\begin{array}{r}81.2 \\
4.4\end{array}$ & $\begin{array}{r}80.5 \\
4.3\end{array}$ & $\begin{array}{r}78.3 \\
4.2\end{array}$ & & \\
\hline Change in public sector debt & -0.9 & -2.5 & -3.2 & -2.5 & -0.1 & 3.5 & -0.5 & -0.7 & -2.1 & -3.0 & -3.0 \\
\hline Identified debt-creating flows $(4+7+12)$ & -4.3 & -3.1 & -4.5 & -5.3 & 4.0 & 5.4 & -0.3 & 0.0 & -1.1 & -1.9 & -1.9 \\
\hline Primary deficit & 1.2 & 1.5 & 0.7 & -0.8 & 5.7 & 5.6 & 4.0 & 3.3 & 2.0 & 1.4 & 1.4 \\
\hline Revenue and grants & 19.5 & 19.6 & 21.2 & 23.1 & 20.7 & 20.5 & 21.0 & 21.5 & 22.1 & 22.7 & 23.2 \\
\hline Primary (noninterest) expenditure & 20.6 & 21.2 & 21.9 & 22.3 & 26.4 & 26.1 & 25.0 & 24.9 & 24.1 & 24.1 & 24.5 \\
\hline Automatic debt dynamics $2 /$ & -5.2 & -4.7 & -5.5 & -5.2 & -2.4 & -1.7 & -4.3 & -3.3 & -3.1 & -3.3 & -3.2 \\
\hline Contribution from interest rate/growth differential 3/ & -4.9 & -4.9 & -5.4 & -4.7 & -3.5 & -1.7 & -4.3 & -3.3 & -3.1 & -3.3 & -3.2 \\
\hline Of which contribution from real interest rate & 1.5 & 2.2 & 1.6 & 1.7 & 0.7 & 2.8 & 1.4 & 2.3 & 2.5 & 2.4 & 2.2 \\
\hline Of which contribution from real GDP growth & -6.3 & -7.1 & -7.1 & -6.4 & -4.2 & -4.6 & -5.7 & -5.6 & -5.6 & -5.6 & -5.4 \\
\hline Contribution from exchange rate depreciation $4 /$ & -0.3 & 0.2 & -0.1 & -0.5 & 1.0 & & $\ldots$ & & $\ldots$ & & \\
\hline Other identified debt-creating flows & -0.3 & 0.0 & 0.4 & 0.7 & 0.7 & 1.5 & 0.0 & 0.0 & 0.0 & 0.0 & 0.0 \\
\hline Privatization receipts (negative) & -0.4 & -0.2 & -0.1 & 0.0 & -0.1 & 0.0 & 0.0 & 0.0 & 0.0 & 0.0 & 0.0 \\
\hline Recognition of implicit or contingent liabilities & 0.1 & 0.3 & 0.4 & 0.7 & 0.8 & 1.5 & 0.0 & 0.0 & 0.0 & 0.0 & 0.0 \\
\hline Other (specify, e.g., bank recapitalization) & 0.0 & 0.0 & 0.0 & 0.0 & 0.0 & 0.0 & 0.0 & 0.0 & 0.0 & 0.0 & 0.0 \\
\hline Residual, including asset changes $(2-3) 5 /$ & 3.4 & 0.6 & 1.3 & 2.8 & -4.0 & -1.9 & -0.1 & -0.8 & -1.0 & -1.1 & -1.2 \\
\hline Public sector debt-to-revenue ratio $1 /$ & 444.7 & 428.0 & 382.1 & 338.3 & 378.0 & 399.3 & 387.0 & 373.7 & 354.5 & 332.2 & 312.0 \\
\hline $\begin{array}{l}\text { Gross financing need 6/ } \\
\text { in billions of U.S. dollars }\end{array}$ & $\begin{array}{r}8.4 \\
58.8\end{array}$ & $\begin{array}{l}10.7 \\
86.5\end{array}$ & $\begin{array}{r}9.9 \\
90.4\end{array}$ & $\begin{array}{r}8.9 \\
104.3\end{array}$ & $\begin{array}{r}15.3 \\
176.5\end{array}$ & $\begin{array}{r}14.5 \\
183.6\end{array}$ & $\begin{array}{r}12.9 \\
178.0\end{array}$ & $\begin{array}{r}11.0 \\
166.1\end{array}$ & $\begin{array}{r}9.3 \\
153.2\end{array}$ & $\begin{array}{r}8.3 \\
151.3\end{array}$ & $\begin{array}{r}7.9 \\
157.5\end{array}$ \\
\hline $\begin{array}{l}\text { Scenario with key variables at their historical averages } 7 / \\
\text { Scenario with no policy change (constant primary balance) in 2009/10-2014/15 }\end{array}$ & & & & & & $\begin{array}{l}81.7 \\
81.7\end{array}$ & $\begin{array}{l}81.6 \\
82.1\end{array}$ & $\begin{array}{l}80.9 \\
83.3\end{array}$ & $\begin{array}{l}79.9 \\
84.3\end{array}$ & $\begin{array}{l}78.9 \\
85.0\end{array}$ & $\begin{array}{l}77.9 \\
85.4\end{array}$ \\
\hline \multicolumn{12}{|l|}{ Key Macroeconomic and Fiscal Assumptions Underlying Baseline } \\
\hline Real GDP growth (in percent) & 8.3 & 9.3 & 9.7 & 9.1 & 6.1 & 6.4 & 8.0 & 7.7 & 7.8 & 8.1 & 8.1 \\
\hline Average nominal interest rate on public debt (in per & 8.0 & 7.5 & 7.7 & 7.7 & 7.7 & 7.9 & 8.1 & 7.8 & 7.7 & 7.6 & 7.5 \\
\hline Average real interest rate (nominal rate minus change in GDP deflator, in percent) & 2.4 & 3.3 & 2.7 & 2.8 & 1.4 & 4.2 & 2.4 & 3.5 & 3.8 & 3.7 & 3.6 \\
\hline Nominal appreciation (increase in US dollar value of local currency, in percent) & 4.6 & -3.3 & 1.9 & 12.3 & -18.7 & & & & & & \\
\hline Inflation rate (GDP deflator, in percent) & 5.6 & 4.2 & 5.0 & 4.9 & 6.2 & 3.7 & 5.7 & 4.3 & 3.9 & 3.9 & 3.9 \\
\hline Growth of real primary spending (deflated by GDP deflator, in percent) & 3.3 & 12.3 & 13.3 & 11.4 & 25.1 & 5.2 & 3.6 & 7.1 & 4.4 & 8.0 & 10.2 \\
\hline Primary deficit & 1.2 & 1.5 & 0.7 & -0.8 & 5.7 & 5.6 & 4.0 & 3.3 & 2.0 & 1.4 & 1.4 \\
\hline
\end{tabular}

Source: Fund staff estimates.

$1 /$ General government debt covers central and state governments, inclusive of MSS bonds.

2/ Derived as $[(r-\pi(1+g)-g+\alpha \varepsilon(1+r)] /(1+g+\pi+g \pi))$ times previous period debt ratio, with $r=$ interest rate; $\pi=$ growth rate of GDP deflator; $g=$ real GDP growth rate; $\alpha=$ share of

foreign-currency denominated debt; and $\varepsilon=$ nominal exchange rate depreciation (measured by increase in local currency value of U.S. dollar).

$3 /$ The real interest rate contribution is derived from the denominator in footnote $2 /$ as $r-\pi(1+g)$ and the real growth contribution as $-g$.

4/ The exchange rate contribution is derived from the numerator in footnote $2 /$ as $\alpha \varepsilon(1+r)$.

$5 /$ For projections, this line includes exchange rate changes.

6/ Defined as public sector deficit, plus amortization of medium and long-term public sector debt, plus short-term debt at end of previous period.

7/ The key variables include real GDP growth; real interest rate; and primary balance in percent of GDP.

$8 /$ Derived as nominal interest expenditure divided by previous period debt stock.

9/ Assumes that key variables (real GDP growth, real interest rate, and other identified debt-creating flows) remain at the level of the last projection year.

CInternational Monetary Fund. Not for Redistribution 


\section{AnNex II: IndiA-Medium-TeRm External Debt Sustainability ANALYSIS}

Figure II.1. India: External Debt Sustainability: Bound Tests 1/

(External debt, in percent of GDP)
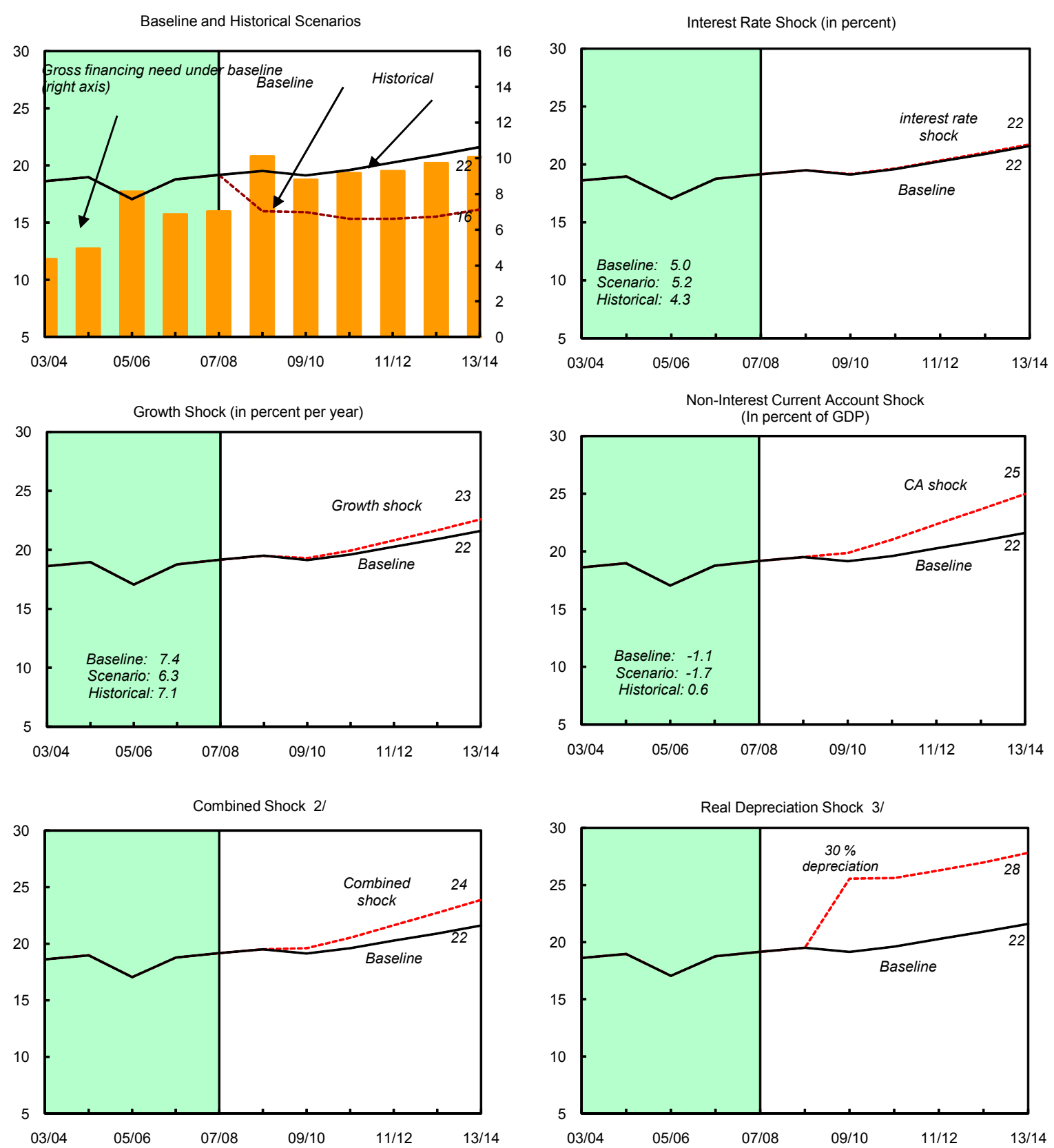

Source: Fund staff estimates.

1/ Shaded areas represent actual data. Individual shocks are permanent one-half standard deviation shocks. Figures in the boxes represent average projections for the respective variables in the baseline and scenario being presented. Ten-year historical average for the variable is also shown.

2/ Permanent 1/4 standard deviation shocks applied to real interest rate, growth rate, and current account balance.

$3 /$ One-time real depreciation of 30 percent occurs in 2007/08. 
Table II.1. India: External Debt Sustainability Framework, 2004/05-2014/15

(In percent of GDP, unless otherwise indicated)

\begin{tabular}{|c|c|c|c|c|c|c|c|c|c|c|c|c|}
\hline \multirow[b]{2}{*}{ Baseline: external debt } & \multicolumn{5}{|c|}{ Actual } & \multicolumn{6}{|c|}{ Projections } & \multirow{2}{*}{ 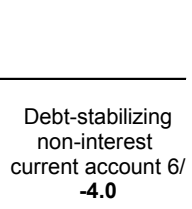 } \\
\hline & 19.0 & 17.0 & 18.8 & 19.2 & 19.5 & 19.1 & 19.6 & 20.3 & 20.9 & 21.6 & 22.5 & \\
\hline Change in external debt & 0.4 & -1.9 & 1.7 & 0.4 & 0.3 & -0.4 & 0.5 & 0.7 & 0.6 & 0.7 & 0.9 & \\
\hline Identified external debt-creating flows $(4+8+9)$ & -4.1 & -3.2 & -2.5 & -6.2 & 1.1 & -2.5 & -1.9 & -2.1 & -2.3 & -2.7 & -2.8 & \\
\hline Imports & 20.9 & 23.6 & 25.7 & 26.5 & 29.9 & 24.4 & 25.1 & 25.0 & 25.3 & 25.7 & 26.3 & \\
\hline Net nondebt creating capital inflows (negative) & -1.9 & -1.9 & -1.6 & -3.8 & -0.3 & -3.3 & -2.7 & -2.5 & -2.6 & -2.9 & -2.9 & \\
\hline Net foreign direct investment, equity & 0.5 & 0.4 & 0.8 & 1.3 & 1.5 & 1.2 & 1.3 & 1.3 & 1.3 & 1.5 & 1.5 & \\
\hline Net portfolio investment,equity & 1.3 & 1.5 & 0.8 & 2.5 & -1.2 & 2.0 & 1.4 & 1.2 & 1.3 & 1.4 & 1.4 & \\
\hline Automatic debt dynamics $1 /$ & -1.9 & -1.9 & -1.4 & -3.1 & -0.4 & -0.6 & -0.8 & -0.6 & -0.4 & -0.4 & -0.6 & \\
\hline Denominator: $1+g+r+g r$ & 1.2 & 1.2 & 1.1 & 1.3 & 1.0 & 1.1 & 1.1 & 1.1 & 1.1 & 1.1 & 1.1 & \\
\hline Contribution from nominal interest rate & 0.7 & 0.6 & 0.6 & 0.7 & 0.8 & 0.5 & 0.6 & 0.8 & 1.0 & 1.1 & 1.0 & \\
\hline Contribution from real GDP growth & -1.3 & -1.5 & -1.5 & -1.3 & -1.2 & -1.1 & -1.4 & -1.4 & -1.4 & -1.5 & -1.6 & \\
\hline Contribution from price and exchange rate changes $2 /$ & -1.3 & -1.0 & -0.5 & -2.5 & & & & & & & & \\
\hline \multicolumn{13}{|l|}{ Scenario with key variables at their historical averages $5 /$} \\
\hline \multicolumn{13}{|l|}{ Key macroeconomic assumptions underlying baseline } \\
\hline Real GDP growth at market prices (in percent) & 8.3 & 9.3 & 9.7 & 9.1 & 6.1 & 6.3 & 8.0 & 7.7 & 7.8 & 8.1 & 8.1 & \\
\hline GDP deflator in US dollars (change in percent) & 7.9 & 5.7 & 2.7 & 17.8 & -7.1 & 4.7 & 0.5 & 1.4 & 1.8 & 1.9 & 1.1 & \\
\hline Nominal external interest rate (in percent) & 4.2 & 3.9 & 4.0 & 4.8 & 4.0 & 2.9 & 3.4 & 4.6 & 5.7 & 5.8 & 4.8 & \\
\hline Growth of exports goods and services (U.S. dollar te & 37.9 & 26.7 & 24.5 & 26.4 & 7.9 & -9.4 & 12.3 & 12.0 & 12.6 & 13.5 & 13.3 & \\
\hline Growth of imports goods and services (U.S & 51.7 & 30.5 & 22.7 & 32.1 & 11.5 & -10.4 & 12.9 & 8.8 & 10.8 & 12.1 & 11.8 & \\
\hline Current account balance, excluding interest payments & 0.3 & -0.6 & -0.4 & -0.8 & -1.8 & -1.4 & -1.6 & -0.9 & -0.7 & -0.6 & -0.7 & \\
\hline Net non-debt creating capital inflows & 1.9 & 1.9 & 1.6 & 3.8 & 0.3 & 3.3 & 2.7 & 2.5 & 2.6 & 2.9 & 2.9 & \\
\hline \multicolumn{13}{|l|}{ B. Bound Tests } \\
\hline \multirow{3}{*}{\multicolumn{5}{|c|}{$\begin{array}{l}\text { B1. Nominal interest rate is at historical average plus one standard deviation } \\
\text { B2. Real GDP growth is at historical average minus one standard deviations } \\
\text { B3. Non-interest current account is at historical average minus one standard deviations } \\
\text { B4. Combination of B1-B3 using } 1 / 2 \text { standard deviation shocks } \\
\text { B5. One time } 30 \text { percent real depreciation in } 2010\end{array}$}} & 19.5 & 19.2 & 19.7 & 20.4 & 21.0 & 21.7 & 22.7 & -3.8 \\
\hline & & & & & 19.5 & 19.3 & 19.9 & 20.8 & 21.7 & 22.6 & 23.8 & -3.8 \\
\hline & & & & & 19.5 & 19.9 & 21.0 & 22.4 & 23.7 & 25.0 & 26.5 & -4.0 \\
\hline
\end{tabular}

Source: Fund staff estimates.

$1 /$ Derived as $[r-g-\rho(1+g)+$ ea $(1+r)](1+g+\rho+g \rho)$ times previous period debt stock, with $r=$ nominal effective interest rate on external debt; $\rho=$ change in domestic GDP deflator in U.S. dollar terms, $g=$ real GDP growth

$2 /$ The contribution from price and exchange rate changes is defined as $[-p(1+g)+e a(1+r)] /(1+g+\rho+g \rho)$ times previous period debt stock. $\rho$ increases with an appreciating domestic currency $(e>0)$ and rising inflation $(b a s e d$ on GDP deflator).

$3 /$ For projection, line includes the impact of price and exchange rate changes.

current account deficit, plus amortization on medium- and long-term debt, plus short-term debt at end of previous period. Short-term debt includes all the outstanding non-resident deposit accounts.

5/ The key variables include real GDP growth; nominal interest rate; dollar deflator growth; and both non-interest current account and non-debt inflows in percent of GDP.

projection year.

(CInternational Monetary Fund. Not for Redistribution 
ANNEX III: INDIA-FUND RELATIONS

(As of November 30, 2009)

I. Membership Status: Joined 12/27/45; Article VIII.

II. General Resources Account

SDR Million

$4,158.20$

$3,246.24$

912.06

Fund holdings of currency

III. SDR Department:

Net cumulative allocation

Holdings
SDR Million

$3,978.26$

$3,297.23$
\% Quota

100.00

78.07

21.93

$\%$ Allocation

100.00

82.88

IV. Outstanding Purchases and Loans: None

V. Financial Arrangements:

$\begin{array}{lcccc}\text { Type } & \begin{array}{c}\text { Approval } \\ \text { Date }\end{array} & \begin{array}{c}\text { Expiration } \\ \text { Date }\end{array} & \begin{array}{c}\text { Amount } \\ \text { Approved } \\ \text { (SDR million) }\end{array} & \begin{array}{c}\text { Amount } \\ \text { Drawn } \\ \text { (SDR million) }\end{array} \\ \text { Stand-By } & 10 / 31 / 1991 & 06 / 30 / 1993 & 1,656.00 & 1,656.00 \\ \text { Stand-By } & 01 / 18 / 1991 & 04 / 17 / 1991 & 551.93 & 551.93 \\ \text { EFF } & 11 / 09 / 1981 & 05 / 01 / 1984 & 5,000.00 & 3,900.00\end{array}$

VI. Projected Obligations to Fund (SDR million; based on existing use of resources and present holdings of SDRs):

\begin{tabular}{lcccr} 
& \multicolumn{5}{c}{ Forthcoming } \\
& $\mathbf{2 0 1 0}$ & $\mathbf{2 0 1 1}$ & $\mathbf{2 0 1 2}$ & $\mathbf{2 0 1 3}$ \\
\cline { 2 - 5 } & & & & \\
Charges/interest & 1.94 & 1.93 & 1.93 & 1.93 \\
Total & 1.94 & 1.93 & 1.93 & 1.93
\end{tabular}

\section{Exchange Rate Arrangement:}

Since March 1, 1993, the Indian rupee has floated against other currencies, although the Reserve Bank of India intervenes in the market periodically. As per the Annual Report on Exchange Arrangements and Exchange Restrictions (AREAER), the exchange rate in India is classified as floating with no pre-announced path for the exchange rate. On August 20,1994, India accepted the obligations of Article VIII, Sections 2, 3, and 4 of the IMF Articles of Agreement. India maintains the following restrictions on the making of payments and transfers for current international transactions, which are subject to Fund approval under Article VIII, Section 2(a): restrictions related to the nontransferability of balances under the India-Russia debt agreement; restrictions arising from unsettled balances under inoperative bilateral payments arrangements with two Eastern European countries; and a restriction on the transfer of amortization payments on loans by non-resident relatives. These restrictions have not been approved by the Executive Board.

\section{Article IV Consultation:}

The previous Article IV consultation discussions were held in December 2008. The staff report (IMF Country Report No. 09/187) was discussed by the Executive Board on February 6, 2009. 
IX. ROSCs:

The data model of the ROSC (IMF Country Report No. 04/96) was issued in April 2004; a fiscal transparency ROSC (available at http://www.imf.org/external/np/rosc/ind/fiscal.htm) was issued in February 2001.

\section{Technical Assistance:}

\section{Department Purpose}

Date of Delivery

MAE

Government securities market

$2 / 94$

MAE

Foreign exchange market

$2 / 95$

FAD

Expenditure control

$5 / 95$

FAD

Public expenditure management

$8 / 95$

FAD

Public expenditure management (follow-up)

$5 / 96$

Government securities market (follow-up)

$7 / 96$

SDDS and statistics

$12 / 96$

STA

Balance of payments statistics

$12 / 97$

SDDS and statistics

$2 / 98$

State level fiscal database and debt register

$11 / 04$

Pilot study on public private partnerships

$12 / 04$

Balance of payments statistics

$9 / 05$

AML/CFT

$5 / 08$

AML/CFT

$10 / 08$

XI.

Outreach and Other Activities:

Department

Purpose

Date of Delivery

OAP/APD/

Conference: A Tale of Two Giants: India's and China's Experience with Reform and Growth

$11 / 03$

NCAER

FAD

Conference: International Experiences with Fiscal Reform

$1 / 04$

APD/FAD

Seminar: Decentralization: International Experiences with Subnational Debt Controls

$1 / 04$

Training: Applying Debt Sustainability Templates to Indian States

$3 / 04$

Training: Revenue Forecasting

$5 / 05$

APD

Seminar: Going Global: India's Emerging Role in the World Economy at Centro di Studi Internazionali sull'Economia e lo Sviluppo

$6 / 06$

Book: India Goes Global: Its Expanding Role in the World Economy

$8 / 06$

Brookings Institution Panel: Is India's High Growth Sustainable?

$4 / 07$

Book: India: Managing Financial Integration and Growth

$6 / 08$

APD

Seminar: "Have We Seen this Movie Before? Comparing The Crisis of 2008 with East Asia 1998", presented at ICRIER/IM-Welt conference

and Yale Initiative on Asian and International Relations

\section{Resident Representative:}

A resident representative's office was opened in November 1991. Mr. Sanjaya Panth has been Senior Resident Representative since August 2008. 


\section{ANNEX IV: INDIA-RELATIONS WITH THE WORLD BANK GROUP}

In Bank FY2009 (July 1-June 30), IBRD/IDA lending totaled \$2.24 billion, slightly above the \$2.15 billion in FY2008. IFC committed \$947 million in FY2009, down slightly from \$1.05 billion in FY2008. The World Bank Group's (WBG) Country Strategy (CAS) for India for 2009-2012 focuses on helping India to fast-track the development of much-needed infrastructure and to support the seven poorest states in achieving higher standards of living for their people. The strategy envisages a total proposed lending program of US $\$ 14.4$ billion for the next three years, of which US\$9.6 billion is from the International Bank for Reconstruction and Development (IBRD) and US $\$ 5$ billion equivalent (SDRs 3.15 billion at the current exchange rate) from the International Development Association (IDA). The projected total lending for FY2009-2012 includes approximately \$3 billion additional IBRD financing in response to the financial crisis.

The overarching objective of the CAS is to scale up the development impact of Bank Group assistance to help India achieve rapid, inclusive growth and sustainable development, and improving service delivery. It aims to do all this while strengthening weaknesses in project implementation, improving the effectiveness of public spending, and achieving demonstrable results to scale up the impact of World Bank assistance.

The diversity of India calls for a differentiated, tailored approach. In India's low-income states and lagging regions in more advanced states, the focus is on achieving the MDGs, relying primarily on IDA resources and non-lending technical assistance (TA). In more advanced states and at the central level, the focus is on strengthening institutions so that they can deal with emerging middleincome challenges, relying on IBRD lending and cutting-edge analytical work. IFC's strategy supports inclusive growth by increasing access to infrastructure and finance, focusing on low-income, rural, and fragile regions and making climate change central to its work.

Implementation of the current CAS has focused on consolidating the program, moving away from many small and fragmented projects towards large-scale programs. The shift to a more substantial engagement has raised the visibility and influence of the Bank Group, while creating opportunities for greater focus on public policy and institutional reform. Progress in implementing this approach is evidenced by the new multi-billion dollar requests from the Government to support projects in key sectors, such as highways, urban development, Ganga River clean-up, rural roads and livelihoods programs.

Going forward, the Bank Group's country strategy will have to adjust further to leverage other financing sources as a result of the limits on Bank Group financing in India. IFC is close to meeting its exposure limit and the Bank is quickly approaching the IBRD single borrower limit. 
Financial operations since 2002/03 are summarized below.

India: World Bank Group Financial Operations

(In millions of U.S. dollars) ${ }^{1 /}$

\begin{tabular}{lrrrrrrr}
\hline & $2002 / 03$ & $2003 / 04$ & $2004 / 05$ & $2005 / 06$ & $2006 / 07$ & $2007 / 08$ & $2008 / 09$ \\
\hline Commitments 2/ & 2,092 & 1,328 & 2,705 & 1,886 & 2,451 & 3,174 & 1,965 \\
IBRD & 951 & 698 & 1,463 & 1,241 & 1,566 & 1,932 & 706 \\
IDA & 1,141 & 630 & 1,242 & 645 & 855 & 1,242 & 1,259 \\
Disbursements & 1,533 & 1,816 & 1,835 & 2,135 & 1,935 & 1,904 & 2,076 \\
IBRD & 647 & 892 & 818 & 938 & 947 & 1,092 & 979 \\
IDA & 886 & 924 & 1,017 & 1,197 & 988 & 812 & 1,097 \\
Repayments & 3,491 & 2,403 & 784 & 842 & 946 & 1,086 & 1,173 \\
IBRD & 3,031 & 1,871 & 201 & 221 & 282 & 363 & 410 \\
IDA & 460 & 532 & 582 & 621 & 664 & 723 & 763 \\
Debt outstanding and disbursed & 26,243 & 27,019 & 28,527 & 28,925 & 30,911 & 33,552 & 32,375 \\
IBRD & 5,082 & 4,238 & 4,865 & 5,557 & 6,277 & 7,040 & 7,584 \\
IDA & 21,161 & 22,781 & 23,662 & 23,368 & 24,634 & 26,512 & 24,790 \\
\hline
\end{tabular}

Source: World Bank.

1/ On an Indian fiscal year basis beginning April 1.

2/ Based on loan approval date. 


\section{ANNEX V: INDIA-RELATIONS WITH the Asian DEVELOPMENT BANK}

The Asian Development Bank (AsDB) operations in India began in 1986. Cumulative public sector loan commitments totaled $\$ 20.8$ billion as of 31 December 2009 for 127 loans. With an additional $\$ 1.7$ billion in private sector loans (the latter without government guarantee), total loan commitments on a cumulative basis amount to $\$ 22.5$ billion. These funds have been provided from the Bank's ordinary capital resources (OCR). Also, AsDB has approved equity investments amounting to $\$ 0.3$ billion. AsDB's lending and equity activities are summarized below.

India: Asian Development Bank Financial Operations

(In millions of U.S. dollars, as of 31 December 2009)

\begin{tabular}{rrrr}
\hline $\begin{array}{c}\text { Calendar } \\
\text { Year }\end{array}$ & $\begin{array}{c}\text { OCR Loan } \\
\text { Commitments }\end{array}$ & $\begin{array}{c}\text { Private } \\
\text { Equity }\end{array}$ & Disbursements \\
\hline $1986-90$ & $2,317.6$ & 10.9 & 338.7 \\
$1991-95$ & $3,364.0$ & 59.5 & $2,131.3$ \\
1996 & 763.0 & -- & 591.7 \\
1997 & 563.0 & 15.5 & 645.0 \\
1998 & 250.0 & -- & 620.4 \\
1999 & 625.0 & -- & 605.3 \\
2000 & 1330.0 & -- & 487.0 \\
2001 & $1,500.0$ & -- & 269.9 \\
2002 & $1,163.6$ & 15.0 & 676.5 \\
2003 & $1,411.0$ & 0.7 & 381.4 \\
2004 & $1,200.0$ & 29.7 & 641.0 \\
2005 & 367.3 & 15.0 & 701.4 \\
2006 & $1,260.0$ & 67.6 & $1,363.5$ \\
2007 & $1,232.1$ & -- & $1,507.7$ \\
2008 & $1,777.6$ & 18.6 & $1,338.9$ \\
2009 & $1,711.0$ & 40.0 & $12,857.7$ \\
Total & $20,835.2$ & 272.3 & \\
\hline
\end{tabular}

Source: Asian Development Bank.

AsDB's India Country Partnership Strategy (2009-2012) (CPS) is based on four strategic pillars: (i) Support for the process of inclusive and environmentally sustainable growth; (ii) Catalyzing investment through the use of innovative business and financing modalities; (iii) Strengthening the results orientation of project design and implementation and emphasizing knowledge solutions; and (iv) Support for regional cooperation. The CPS has been designed to support Government of India's efforts in facilitating inclusive growth and speeding up the pace of poverty reduction and social development as emphasized in the Eleventh Five-Year Plan (2007-2012). It has been prepared within a results-based framework, and aims at significantly strengthening AsDB support for infrastructure development in the relatively poorer states of India, promoting public private partnerships in infrastructure, supporting climate change adaptation and mitigation, and encouraging the use of innovative financing modalities (non-sovereign loans and cofinancing) to enhance the leverage of AsDB operations. 


\section{ANNEX VI: INDIA-STATISTICAL ISSUES}

1. Macroeconomic statistics are adequate for surveillance, but weaknesses remain in the timeliness and coverage of certain statistical series. India has an elaborate system for compiling economic and financial statistics and produces a vast quantity of data covering most sectors of the economy. India subscribed to the Special Data Dissemination Standards (SDDS) on December 27, 1996 and started posting its metadata on the Dissemination Standards Bulletin Board on October 30,1997. It is currently in observance of the SDDS, although it uses flexibility options for timeliness of data on general government operations and on the periodicity and timeliness of labor market data.

2. The data module of the Report on Observance of Standards and Codes (ROSC, IMF Country Report No. 04/96) was published in April 2004. It assesses India's data dissemination practices against the SDDS requirements and assesses the quality of six datasets based on the Data Quality Assessment Framework (DQAF) developed by STA.

3. National accounts and employment statistics: The Central Statistical Organization (CSO) has recently reduced the dissemination lag for quarterly releases from three to two months and released a new series of national accounts, with base year 1999-2000 in February 2006. Estimates of value added in constant prices for public administration and defense may be biased upwards, as they are based on the government's wage bill (with arrears counted in the year that they are paid) deflated by the Wholesale Price Index (WPI). Comprehensive data on employment are only available on an annual basis and with a substantial lag. Also, employment data are only produced for the formal sector, which accounts for a small segment of the labor market.

4. Price statistics: The consumer price indices (CPIs) are based on weights that are over ten years old and do not fully capture price developments in the economy. However, since January 2006, the Labour Bureau has published a revised CPI for industrial workers with a 2001 base year and a Working Group is engaged in the revision of the current producer price index to a new base. Presently, there are four CPIs, each based on the consumption basket of a narrow category of consumers (namely industrial workers, urban and nonmanual employees, agricultural laborers, and rural laborers). The CPIs are published with a lag of about one month. A WPI (1993/94=100) is published weekly with a lag of two weeks and is subject to large revisions, especially in periods of rising inflation. In addition, the representativeness of the index may be undermined by the collection of prices from a relatively small sample of products and the infrequent updating of weights. Real estate and housing price data are not available on a timely basis and the geographic coverage is limited.

5. External sector statistics: While the concepts and definitions used to compile balance of payments statistics are broadly in line with the fifth edition of the Balance of Payments Manual (BPM5), the RBI presentation does not strictly follow the BPM5. Furthermore, trade data have quality, valuation, timing, and coverage problems, and data on trade prices, volumes, and composition are not regularly available on a timely basis. Only trade credit extended for more than 180 days is included in the balance of payments (and the IIP and external debt data); trade credit is often less than 180 days in most countries. Bilateral data on services exports to the United States and other developed countries are manifold higher than counterpart services imports published by these same countries. External debt statistics are available on a quarterly basis with a one quarter lag. Estimates of short-term external debt are presented in the debt statistics on an original maturity basis. The short-term maturity attribution on a residual maturity basis is only available annually (and excludes residual maturity of medium- and long-term nonresident Indian accounts). The international investment position (IIP) statistics cover the sectors prescribed in the BPM5 and these data are 
disseminated within six months of the reference period in respect of annual data. Coverage of direct investment positions data is hampered by the absence of appropriate legal or institutional authority. India began disseminating the Data Template on International Reserves and Foreign Currency Liquidity as prescribed under the SDDS in December 2001. The more up-to-date information on certain variables, such as total foreign reserves, foreign currency assets, gold, and SDRs, are available on a weekly basis and are disseminated as part of a weekly statistical supplement on the RBI web site.

6. Monetary and financial statistics: The RBI web site and the RBI Bulletin publish a wide array of monetary and financial statistics, including reserve money and its components, RBl's survey, monetary survey, liquidity aggregates (outstanding amounts), interest rates, exchange rates, foreign reserves, and results of government securities auctions. The frequency and quality of data dissemination have improved substantially in recent years.

7. Concepts and definitions used by the RBI to compile monetary statistics are in broad conformity with the guidelines provided in the Monetary and Financial Statistics Manual (MFSM). Nevertheless, the following concepts and principles deviate from the MFSM. First, the resident sector data do not provide sufficient information on the sectoral distribution of domestic credit. Specifically, under their present sectorization scheme, the authorities subdivide the resident nonbank sector data by (i) central government; (ii) state government; and (iii) the commercial sector (including other financial corporations, public and other nonfinancial corporations, and other resident sectors). Second, commercial banks add accrued interest to credit and deposit positions on a quarterly basis only (instead of the prescribed monthly basis).

8. The RBI reports monetary data for IFS on a regular basis. Since October 2006, the RBI has initiated the electronic reporting of monetary data, which is a major improvement from the previous paper-based reporting which was prone to errors and delays. India has also submitted to STA test data (starting from December 2001 data) on the Standardized Report Forms (SRFs) that have been developed to implement the methodology outlined in the MFSM. STA is working with the authorities in resolving the outstanding data issues on the development of the SRFs.

9. Fiscal operations: The Ministry of Finance (MoF) posts selected central government monthly fiscal data and quarterly debt data on its web site. However, no monthly data on fiscal performance at the state level are available, and annual data are available only with an 8-month to 10-month lag. Consolidated information is unavailable on local government operations. In addition, data on the functional and economic classification of expenditures are available with considerable lag. There is also scope to improve the analytical usefulness of the presentation of the fiscal accounts. For example, classification of government expenditure between developmental/nondevelopmental and plan/nonplan obscures the economic nature and impact of fiscal actions. The MoF reports central government data (on a cash basis) for publication in the Government Finance Statistics Yearbook (latest reported data correspond to 2006). Some limited general government data has been reported for 2002. 


\section{India-Table of Common Indicators Required for Surveillance}

As of December 18, 2009

\begin{tabular}{|c|c|c|c|c|c|c|c|}
\hline & \multirow[b]{2}{*}{$\begin{array}{c}\text { Date of } \\
\text { latest } \\
\text { observation }\end{array}$} & \multirow[b]{2}{*}{$\begin{array}{l}\text { Date } \\
\text { received }\end{array}$} & \multirow[b]{2}{*}{$\begin{array}{c}\text { Frequency } \\
\text { of } \\
\text { Data 1/ }\end{array}$} & \multirow[b]{2}{*}{$\begin{array}{l}\text { Frequency } \\
\text { of } \\
\text { Reporting 1/ }\end{array}$} & \multirow[b]{2}{*}{$\begin{array}{l}\text { Frequency } \\
\text { of Publi- } \\
\text { cation 1/ }\end{array}$} & \multicolumn{2}{|c|}{ Memo Items 2/ } \\
\hline & & & & & & $\begin{array}{l}\text { Data Quality- } \\
\text { Methodological } \\
\text { soundness 2/ }\end{array}$ & $\begin{array}{l}\text { Data Quality- } \\
\text { Accuracy and } \\
\text { reliability 3/ }\end{array}$ \\
\hline Exchange rates & $12 / 18 / 09$ & $12 / 18 / 09$ & $\mathrm{D}$ & $\mathrm{D}$ & $\mathrm{D}$ & & \\
\hline $\begin{array}{l}\text { International reserve assets and reserve } \\
\text { liabilities of the monetary authorities } 4 /\end{array}$ & $12 / 18 / 09$ & $12 / 25 / 09$ & W & W & W & & \\
\hline Reserve/base money & $12 / 18 / 09$ & $12 / 25 / 09$ & W & W & W & O, O, LO, LO & $\mathrm{O}, \mathrm{O}, \mathrm{O}, \mathrm{O}, \mathrm{O}$ \\
\hline Broad money & $12 / 04 / 09$ & $12 / 25 / 09$ & BW & BW & W & & \\
\hline Central bank balance sheet & Mar. 09 & 08/27/09 & A & A & A & & \\
\hline $\begin{array}{l}\text { Consolidated balance sheet of the } \\
\text { banking system }\end{array}$ & Mar. 09 & 09/15/09 & A & A & A & & \\
\hline Interest rates $5 /$ & $12 / 18 / 09$ & $12 / 18 / 09$ & $\mathrm{D}$ & $\mathrm{D}$ & $\mathrm{D}$ & & \\
\hline Consumer price index & Nov. 09 & $12 / 24 / 09$ & M & M & M & O, LNO, O, O & $\begin{array}{l}\text { LNO, LO, O, } \\
\quad \mathrm{O}, \mathrm{O}\end{array}$ \\
\hline $\begin{array}{l}\text { Revenue, expenditure, balance and } \\
\text { composition of financing } 6 / \\
\text { - General Government } 7 /\end{array}$ & Mar. 09 & $09 / 15 / 09$ & $A$ & A & $A$ & LNO, LO, O, O & $\begin{array}{l}\text { O, O, O, O, } \\
\text { LO }\end{array}$ \\
\hline $\begin{array}{l}\text { Revenue, expenditure, balance and } \\
\text { composition of financing } 6 / \\
\text { - Central Government }\end{array}$ & Oct. 09 & $12 / 01 / 09$ & M & M & M & & \\
\hline $\begin{array}{l}\text { Stocks of central government and } \\
\text { central government-guaranteed debt } 8 /\end{array}$ & Mar. 09 & 09/15/09 & A & A & A & & \\
\hline External current account balance & Mar-Jun. 09 & $10 / 08 / 09$ & Q & Q & Q & LO, O, LO, O & $\begin{array}{l}\text { LO, O, O, O } \\
\text { LO }\end{array}$ \\
\hline $\begin{array}{l}\text { Exports and imports of goods and } \\
\text { services }\end{array}$ & Mar-Jun. 09 & $10 / 08 / 09$ & Q & Q & Q & & \\
\hline GDP/GNP & Jul-Sep. 09 & $12 / 01 / 09$ & Q & Q & Q & $\begin{array}{l}\text { LO, LNO, LO, } \\
\text { LO }\end{array}$ & $\begin{array}{l}\text { LNO, LNO, } \\
\text { O, O, LO }\end{array}$ \\
\hline Gross external debt & Mar-Jun. 09 & $12 / 01 / 09$ & $Q$ & $Q$ & $Q$ & & \\
\hline
\end{tabular}

1/ Daily (D), Weekly (W), Biweekly (BW), Monthly (M), Quarterly (Q), Annually (A), Irregular (I); Not Available (NA).

2/ Reflects the assessment provided in the data ROSC (published on April 2, 2004, and based on the findings of the mission that took place during May 13-30, 2002) for the dataset corresponding to the variable in each row. The assessment indicates whether international standards concerning concepts and definitions, scope, classification/sectorization, and basis for recording are fully observed (O), largely observed (LO), largely not observed (LNO), or not observed (NO).

3/ Same as footnote 7, except referring to international standards concerning source data, statistical techniques, assessment and validation of source data, assessment and validation of intermediate data and statistical outputs, and revision studies

4/ Includes reserve assets pledged or otherwise encumbered as well as net derivative positions.

5/ Both market-based and officially-determined, including discount rates, money market rates, rates on treasury bills, notes and bonds.

6/ Foreign, domestic bank, and domestic nonbank financing.

7/ The general government consists of the central government (budgetary funds, extra budgetary funds, and social security funds) and state governments.

$8 /$ Including currency and maturity composition. 


\section{Statement by the IMF Staff Representative on India January 25, 2010}

1. This statement contains information that has become available since the staff report was circulated to the Executive Board. This information does not alter the thrust of the staff appraisal.

\section{Economic and financial developments}

2. Activity indicators have gathered further momentum. Industrial production growth rose to 11.7 percent $y / y$ in November, with both consumer durables and capital goods picking up steam. Export growth also rose sharply, reaching 15 percent $y / y$ in December. The new orders and new export orders components of the manufacturing PMI (both of which are above the expansionary threshold) have risen substantially in December, pointing to a continuation of robust growth in domestic demand and exports.

3. Inflation pressures have intensified. Headline WPI inflation rose to $71 / 3$ percent $y / y$ in December (12 percent in sequential terms, using the 3-month moving average saar), in part because of large food price gains. CPI inflation measures are in the $13 \frac{1}{2}-171 / 4$ percent range, given the higher weight of food in the consumption basket. But core inflation-excluding food and energy — has also continued to accelerate, reaching $52 / 3$ percent in sequential terms.

4. Financial conditions have continued to improve. Net portfolio inflows have surged, reaching US $\$ 3.2$ billion so far in January (compared to a monthly average of US\$2 billion in March-December 2009). Credit growth, which had dipped below 10 percent $y / y$ in October, reached 141/3 percent $y / y$ in December 2009.

\section{Policy developments}

5. On January 14, the government announced several measures to tackle rising food prices by improving the availability of major agricultural products. The government will release 2-3 million tons of wheat and rice in the open market above the allocation made under the public distribution system and has advised states to remove the VAT on imported sugar and to enforce anti-hoarding measures.

\section{In January, the Reserve Bank of India has allowed repos in corporate debt securities and exchange trading of major currencies futures against the rupee.}




\section{INTERNATIONAL MONETARY FUND}

Public Information Notice

EXTERNAL

RELATIONS

DEPARTMENT
Public Information Notice (PIN) No. 10/18

FOR IMMEDIATE RELEASE

February 4, 2010
International Monetary Fund

$70019^{\text {th }}$ Street, NW

Washington, D. C. 20431 USA

\section{IMF Executive Board Concludes 2009 Article IV Consultation with India}

On January 25, 2010, the Executive Board of the International Monetary Fund (IMF) concluded the Article IV consultation with India. ${ }^{1}$

\section{Background}

India's economy is one of the first in the world to recover after the global crisis. Prompt fiscal and monetary easing, combined with the fiscal stimulus already in the pipeline and the return of risk appetite in financial markets, have brought growth close to pre-crisis levels. Leading indicators suggest the output gap will continue to close. Capital inflows are back on the rise, and financial markets have regained most of the lost ground.

Growth is projected to rise from 63/4 percent in 2009/10 (April-March) to 8 percent the following year. Agriculture is likely to contract by about 1 percent in 2009/10 due to the drought, but nonagricultural GDP growth is expected to gather momentum. Private consumption would benefit from better employment prospects and less uncertainty, and investment would be boosted by robust corporate profits, rising business confidence, and favorable financing conditions. With India's long-term prospects remaining strong and private sector balance sheets sound, we expect growth to be back at potential in 2010/11 even if advanced economies grow below trend.

\footnotetext{
${ }^{1}$ Under Article IV of the IMF's Articles of Agreement, the IMF holds bilateral discussions with members, usually every year. A staff team visits the country, collects economic and financial information, and discusses with officials the country's economic developments and policies. On return to headquarters, the staff prepares a report, which forms the basis for discussion by the Executive Board. At the conclusion of the discussion, the Managing Director, as Chairman of the Board, summarizes the views of Executive Directors, and this summary is transmitted to the country's authorities. An explanation of any qualifiers used in summings up can be found here: http://www.imf.org/external/np/sec/misc/qualifiers.htm.
} 
Near-term risks are broadly balanced. On the upside, an acceleration of reforms and capital inflows could spur investment. On the downside, the main risks are elevated inflation and financing constraints arising from-among other things - the fiscal deficit, which could put breaks on the recovery. Tail risks include asset price bubbles and a sudden stop in capital inflows caused by turmoil in global financial markets.

India's medium-term growth prospects remain bright. India was not at the center of the global crisis and growth is well balanced and mainly reliant on domestic drivers. Risks to this favorable outlook stem primarily from difficulties in implementing productivity-enhancing reforms and continued supply bottlenecks.

\section{Executive Board Assessment}

Executive Directors congratulated the authorities on their strong record of sound macroeconomic policies and decisive actions leading to India's early and vigorous recovery from the global crisis. Directors noted that India's rapid recovery has brought fiscal and monetary policy trade-offs to a head earlier than in other countries. Longer-term priorities are financial sector reforms to facilitate infrastructure investment, and fiscal consolidation. Directors were reassured by the authorities' vigilant monitoring and action plans to address the challenges ahead.

Directors commended the Reserve Bank of India $(\mathrm{RBI})$ for starting the first phase of exit from monetary accommodation and generally considered that conditions are ripe for a progressive normalization of the monetary stance. They noted that this will require fine judgments by the $\mathrm{RBI}$, and favored a gradual approach to ensure the recovery reaches its full potential. Given long transmission lags and the low policy rates, most Directors advised a timely start of the withdrawal of monetary stimulus, which would help anchor inflation expectations and soften the impact on long-term interest rates. More extended guidance from the RBI on future inflation may also help in this regard.

Directors recognized the challenges the authorities face in managing capital inflows, and welcomed their intention to continue to employ a range of measures. Most Directors considered that rupee appreciation would help contain inflation and manage capital inflows, although a few Directors argued for caution in this area. Sterilized intervention could help reduce excessive exchange rate volatility, provided it does not generate further inflows. Directors also supported the RBI's approach to use prudential measures in case asset bubbles were to develop. Given the need to develop India's financial markets, several Directors advised a tightening of capital controls only as a last resort. 
Directors noted that India's financial system has weathered the global crisis well. They supported the initiatives to further strengthen the capital of public banks and financial regulation, and the higher provisioning requirements recently introduced. While appreciating India's selfassessment of the financial sector, they noted that the assessment of financial stability could be further strengthened via multifactor stress tests and an independent evaluation of compliance with international standards. Directors also encouraged the authorities to improve mechanisms for dealing with distressed assets and the insolvency framework.

Directors concurred that infrastructure investment is a priority and encouraged the authorities to maintain the momentum of reforms to ensure that the financial system will provide adequate funding. They stressed the importance of developing a vibrant corporate bond market and reforms that would foster greater participation by pension funds and the insurance sector in funding infrastructure. Several Directors also considered that further opening the local debt market to foreign investors could help in enhancing liquidity. A few other Directors advised a more cautious approach in view of the volatility and vulnerabilities associated with capital flows. Directors considered that public institutions have an important role to play in establishing the overall framework for infrastructure financing and in filling market gaps, while carefully managing risks to the public sector.

Directors welcomed the authorities' announcement to lower the deficit starting from the next budget. With the recovery becoming entrenched and given India's high debt, they encouraged the authorities to lay out a concrete strategy for reducing debt through durable reforms, which would boost credibility and foster growth. Most Directors considered that anchoring India's medium-term fiscal framework with a debt target would be helpful.

Directors looked forward to the introduction of the Goods and Services Tax and the new Direct Tax Code. They encouraged the authorities to phase out regressive subsidies and introduce market-based pricing for petroleum products. Over time, the subsidies on products consumed predominantly by the poor should be replaced with targeted support. Directors welcomed the government's focus on raising the quality of public spending and service delivery, and the announcements regarding privatization.

\footnotetext{
Public Information Notices (PINs) form part of the IMF's efforts to promote transparency of the IMF's views and analysis of economic developments and policies. With the consent of the country (or countries) concerned, PINs are issued after Executive Board discussions of Article IV consultations with member countries, of its surveillance of developments at the regional level, of post-program monitoring, and of ex post assessments of member countries with longer-term program engagements. PINs are also issued after Executive Board discussions of general policy matters, unless otherwise decided by the Executive Board in a particular case.
} 
India: Selected Economic Indicators, 2005/06-2010/11 1/

\begin{tabular}{|c|c|c|c|c|c|c|}
\hline & $2005 / 06$ & $2006 / 07$ & $2007 / 08$ & $\begin{array}{r}2008 / 09 \\
\text { Prel. }\end{array}$ & $\begin{array}{r}2009 / 10 \\
\text { Proj. }\end{array}$ & $\begin{array}{r}\text { 2010/11 } \\
\text { Proj. } \\
\end{array}$ \\
\hline \multicolumn{7}{|l|}{ Growth (y/y percent change) } \\
\hline Real GDP (at factor cost) & 9.5 & 9.7 & 9.0 & 6.7 & 6.7 & 8.0 \\
\hline Industrial production & 8.2 & 11.5 & 8.5 & 2.7 & $\ldots$ & $\ldots$ \\
\hline \multicolumn{7}{|l|}{ Prices (y/y percent change, average) } \\
\hline Wholesale prices (1993/94 weights) & 4.4 & 5.4 & 4.7 & 8.3 & 3.1 & 6.4 \\
\hline Wholesale prices (1993/94 weights, end of period) & 4.1 & 6.7 & 7.7 & 0.8 & 8.1 & 5.5 \\
\hline Consumer prices - industrial workers (2001 weights) & 4.4 & 6.7 & 6.2 & 9.1 & 11.2 & 8.8 \\
\hline \multicolumn{7}{|l|}{ Saving and investment (percent of GDP) } \\
\hline Gross saving 2/ & 34.3 & 35.8 & 37.6 & 37.4 & 37.5 & 38.2 \\
\hline Gross investment 2/ & 35.5 & 36.9 & 39.1 & 40.0 & 39.4 & 40.4 \\
\hline \multicolumn{7}{|l|}{ Fiscal position (percent of GDP) $3 /$} \\
\hline Central government deficit & -4.6 & -4.4 & -3.3 & -8.0 & -7.6 & -6.2 \\
\hline General government deficit & -7.2 & -6.3 & -4.7 & -11.0 & -11.2 & -9.4 \\
\hline General government debt 4/ & 84.0 & 80.8 & 78.3 & 78.2 & 81.7 & 81.2 \\
\hline \multicolumn{7}{|l|}{ Money and credit (y/y percent change, end-period) $5 /$} \\
\hline Broad money & 21.2 & 21.5 & 20.9 & 18.8 & 18.7 & $\ldots$ \\
\hline Credit to commercial sector & 32.2 & 25.8 & 20.9 & 17.0 & 11.8 & $\ldots$ \\
\hline \multicolumn{7}{|l|}{ Financial indicators (percent, end-period) $5 /$} \\
\hline 91-day treasury bill yield & 6.1 & 8.0 & 7.2 & 5.0 & 3.3 & $\ldots$ \\
\hline 10-year government bond yield & 7.5 & 8.0 & 7.9 & 7.0 & 7.2 & $\ldots$ \\
\hline Stock market (y/y percent change, end-period) & 73.7 & 15.9 & 19.7 & -37.9 & 86.2 & $\ldots$ \\
\hline \multicolumn{7}{|l|}{ External trade $6 /$} \\
\hline Merchandise exports (US\$ billions) & 105.2 & 128.9 & 166.2 & 175.2 & 145.8 & 178.0 \\
\hline y/y percent change & 23.4 & 22.6 & 28.9 & 5.4 & -16.7 & 22.1 \\
\hline Merchandise imports (US\$ billions) & 157.1 & 190.7 & 257.8 & 294.6 & 259.0 & 310.8 \\
\hline $\mathrm{y} / \mathrm{y}$ percent change & 32.1 & 21.4 & 35.2 & 14.3 & -12.1 & 20.0 \\
\hline Net oil imports (US\$ billions) & 32.3 & 38.3 & 54.8 & 62.7 & 51.6 & 59.2 \\
\hline \multicolumn{7}{|l|}{ Balance of payments (US\$ billions) } \\
\hline Current account balance & -9.9 & -9.6 & -17.0 & -29.8 & -24.0 & -29.5 \\
\hline (in percent of GDP) & -1.2 & -1.0 & -1.5 & -2.6 & -1.9 & -2.1 \\
\hline Foreign direct investment, net & 3.0 & 7.7 & 15.4 & 17.5 & 15.8 & 17.4 \\
\hline Portfolio investment, net (equity and debt) & 12.5 & 7.1 & 29.6 & -14.0 & 25.8 & 19.8 \\
\hline Overall balance & 15.1 & 36.6 & 92.2 & -20.1 & 33.2 & 27.2 \\
\hline \multicolumn{7}{|l|}{ External indicators } \\
\hline Gross reserves (in billions of U.S. dollars, end-period) & 151.6 & 199.2 & 309.7 & 252.0 & 300.7 & 327.9 \\
\hline (In months of imports) $7 /$ & 7.7 & 7.7 & 10.7 & 9.7 & 9.8 & 9.6 \\
\hline External debt (percent of GDP, end-period) & 17.0 & 18.8 & 19.2 & 19.5 & 19.2 & 19.8 \\
\hline Of which: short-term debt $8 /$ & 3.3 & 3.8 & 7.2 & 7.5 & 7.4 & 8.1 \\
\hline Ratio of gross reserves to short-term debt (end-period) 8/ & 5.6 & 5.7 & 3.7 & 2.9 & 3.2 & 2.9 \\
\hline Gross reserves to broad money (percent; end-period) & 24.8 & 26.1 & 31.0 & 26.8 & $\ldots$ & $\ldots$ \\
\hline Debt service ratio 9/ & 10.1 & 4.9 & 5.3 & 5.4 & 5.5 & 6.2 \\
\hline \multicolumn{7}{|l|}{ Real effective exchange rate } \\
\hline (y/y percent change, period average for annual data) & 4.5 & -2.2 & 8.2 & -7.1 & $\cdots$ & $\ldots$ \\
\hline Exchange rate (rupee/US\$, end-period) 5/ & 44.6 & 43.5 & 40.1 & 50.7 & 46.4 & $\ldots$ \\
\hline \multicolumn{7}{|l|}{ Memorandum item (in percent of GDP) } \\
\hline Subsidy related bond issuance $10 /$ & 0.5 & 1.0 & 0.6 & 1.8 & 0.3 & 0.4 \\
\hline
\end{tabular}

Sources: Data provided by the Indian authorities; CEIC Data Company Ltd; Bloomberg L.P.; World Development Indicators; and IMF staff estimates and projections.

$1 /$ Data are for April-March fiscal years.

2/ Differs from official data, calculated with gross investment and current account. Gross investment includes errors and omissions.

3 / Divestment proceeds treated as below-the-line financing. Subsidy related bond issuance included in total expenditure and in the balance.

$4 /$ Includes combined domestic liabilities of the center and the states, inclusive of MSS bonds, and sovereign external debt at year-end exchange rates.

5/ For 2009/10, as of November 2009.

$6 /$ On balance of payments basis.

7/ Imports of goods and services projected over the following twelve months.

8/ Short-term debt on residual maturity basis, including estimated short-term NRI deposits on residual maturity basis.

9 / In percent of current account receipts excluding grants.

10 / Issued by the central government to $\mathrm{FCl}$, the state-owned oil refining/distribution companies, and fertilizer companies as compensation for losses incurred from the provision of subsidies. 


\section{Statement by Arvind Virmani, Executive Director for India and Parthay Ray, Advisor to Executive Director \\ January 25, 2010}

1. Our Indian Authorities appreciate the value of the open and constructive policy dialogue with the Fund. There has been convergence of views between the staff and the Authorities on many issues, and the Authorities' stance on specific aspects of the assessment already finds a place in the staff report. For the sake of emphasis, completeness and clarity comments on some key issues are detailed below.

2. The Indian economy is among the first in the world to recover from the global crisis. While the current GDP growth is still lower than the average annual growth rate of 8.8 per cent during the five-year period from 2003-04 (i.e., April 2003 to March 2004) to 2007-08, challenges remain in respect of a broad-based recovery. While food prices have experienced a spurt, increases in prices of manufactured products (as reflected in the movements of the wholesale price index of manufactured products, year-on-year) have been benign. The recovery has been induced by conventional and unconventional fiscal and monetary-banking policy responses. With the gradual return of normalcy to the global economy, the unwinding of monetary and fiscal measures and their timing remains a challenge. The management of the spurt in portfolio flows, which is now being increasingly recognized for its negative externalities, is a renewed challenge. Financing infrastructure is another area that has drawn attention of Indian policy makers.

\section{Recent Turnaround and Future Expectations}

3. It may be pertinent to note that the Economic Survey, 2008-09 of the Government of India (released in July 2009) stated categorically,

"If the US economy bottoms out by September 2009, there could be good possibility for the Indian economy repeating its 2008-09 performance, i.e. around $7.0 \pm 0.75$ per cent in the fiscal 2009-10 (assuming a normal monsoon). The pattern of fiscal 200809 may be repeated in that case, though in an inverse sequence, with two not so good quarters followed by two good quarters making a 'U'-shaped revival of the growth path" (page 27).

4. In line with these expectations, during the second quarter of 2009-10 (i.e., July September, 2009), the economy rebounded with a real GDP growth of 7.9 percent. In fact, continuation of these trends could make it possible for the economy to grow at $7-7.75$ percent in 2009-10. For the more recent period, industrial production grew by 11.7 percent in November 2009 on a year-on-year basis. Almost all segments of the financial markets continued to remain stable and there has also been a pickup in net capital inflows, particularly from the foreign institutional investors. All these bear testimony to the inherent strength of the economy, experiencing a rapid and strong recovery from the knock-on effects of the global crisis. 
5. Interestingly, the staff projections indicate a deceleration in the growth rate during 2011 - 2013 (Table in page 3). However, our expectation is that in the medium-term, India should be back on the new (post 2003-04) trend growth path of 8.5 to 9 percent per annum, with efforts being geared towards removal of critical policy and institutional bottlenecks.

\section{Return to the Path of Fiscal Consolidation}

6. Welcoming the Authorities' intention to lower the Central Government's deficit, the staff has emphasized discretionary measures. In this context, it may be noted that the path of fiscal consolidation in India has been governed by the Fiscal Responsibility and Budget Management Act (FRBMA), 2003. Since then, India has made impressive reductions in deficits both at the Central Government and the State Government levels. However, as a fallout of the global crisis, and despite some automatic stabilizers, fiscal policy in India, as elsewhere in the world, has played the main role of boosting demand. Consequently, there were a number of fiscal stimulus packages. The fiscal stimulus included both tax relief to boost demand and increased expenditure on public projects to create employment and public assets. These unusual developments during 2008-09 necessitated deviation from the FRBMA. The Budget for 2009-10 has carried forward this policy through a fiscal expansion of 4.1 per cent of GDP (over 2007-08 levels).

7. While such fiscal expansion as a crisis management measure is in line with internationally adopted policies at that juncture, going forward, a strategy of fiscal consolidation will be of paramount importance. The Medium Term Fiscal Policy Statement, 2009-10 has provided the roadmap with the fiscal deficit declining to 5.5 per cent of GDP in 2010-11 and further to 4.0 per cent of GDP in 2011-12. In this process, both revenue augmentation as well as rationalization of expenditure are important. In particular, the reform of the subsidy regime, as envisaged in the Budget for 2009-10, could provide a more sustainable base for fiscal consolidation.

8. In order to further the process of fiscal consolidation initiated through the FRBMA, one of the terms of reference of the Thirteenth Finance Commission (TFC), whose report is expected to be released shortly, has been to assess the impact of various obligations of the Central Government on the deficit targets, and to suggest, " ... a suitably revised roadmap with a view to maintaining the gains of fiscal consolidation through 2010 to 2015." Going forward, the Report of the TFC and the proposed goods and services tax coupled with the initiative on Direct Taxes Code would provide the impetus to a rule-based fiscal consolidation process.

\section{Balance of Payments and Capital Inflows}

9. As per the latest data on India's balance of payments (BoP) for the second quarter (July-September 2009) of 2009-10, while growth in exports and imports continued their declining trend, trade deficit was lower reflecting larger fall in imports, especially oil imports, on account of lower oil prices. Despite lower trade deficit, current account deficit at US\$ 12.6 billion in Q2 of 2009-10 was almost at the same level as last year, mainly on account of lower net invisibles surplus. 
10. As far as capital inflows are concerned, more recent data are available and during the financial year 2009-10 so far, accruals on account of foreign direct investment, deposits of non-resident Indians and portfolio investment were significantly higher as compared to those of the previous year. In particular, investments of Foreign Institutional Investors went up by as much as US \$ 22.8 billion during April 1, 2009 - January 8, 2010, as against a net withdrawal of US $\$ 11.9$ billion during the corresponding period of the previous financial year. The Indian rupee appreciated by 11.24 percent against the US dollar as on January 8 , 2010 over end-March 2009.

11. The volatility of capital flows and the exchange rate has its attendant implications for the monetary policy. Though the consequences of capital inflows during 2009-10 are manageable, macro management could become more challenging as the growth expectations approach the trend ( $8.5-9$ percent) levels leading to more capital inflows. The negative externalities arising from volatility in capital flows will need to be thought through and appropriately addressed.

\section{Monetary Policy Challenges}

12. An important challenge facing India at the current juncture is the build-up of inflationary pressures, particularly in respect of food prices. In this context, the nature of monetary policy response has generated considerable debate in India. First, it is generally agreed that monetary policy is not an efficient instrument for reining in sectoral inflation. In India, prices of primary articles have risen by 14.9 percent, prices of fuel and manufactures rose by 4.3 percent and 5.2 percent, respectively in December 2009 (year-on-year). Accordingly, the overall rate of inflation, which was in negative territory during June-August 2009 and around 1 percent in September-October 2009, has edged above the threshold of 5 percent which is monitored for monetary policy purposes. Second, India like many other EMEs suffered the knock-on effects of the global crisis and in response, monetary and fiscal policies had to switch to an unusually high accommodative stance, including through unconventional modes. Overall GDP growth remains well below the pre-crisis average of 8.8 percent and bank credit growth has decelerated to around 14 percent as against around 24 percent a year ago. Consequently, the trade-off facing monetary policy is sharp - too early an exit from accommodation risks jeopardizing the recovery; too delayed an exit may allow current inflationary pressures to get embedded in inflation expectations. Obviously, a judgment call has to be made, but fully sensitive that either side is weighed down by large risks. So far, on a careful balancing of the trade-off and the existing slack in resource utilization in the economy, the conduct of monetary policy has been supporting the recovery while allowing supply-side management measures to work through and correct shortages in specific commodities.

13. In the face of incipient signs of generalized inflation, it would be an error to assume that monetary policy in India will continue to remain accommodative. Indeed, against the backdrop of developments in sectoral inflation conditions, the October 2009 review of monetary policy affirmed its resolve to "keep a vigil on the trends in inflation and be prepared to respond swiftly and effectively through policy adjustments to stabilise inflation expectations". It further stated: "....it bears emphasis that the Reserve Bank is mindful of its fundamental commitment to price stability. It will continue to monitor the price situation in 
its entirety and will take measures as warranted by the evolving macroeconomic conditions swiftly and effectively." Furthermore, it is important to note that a calibrated withdrawal of accommodation has already commenced in the form of restoring of secondary reserve requirements (the Statutory Liquidity Ratio) to 25 percent (after a temporary lowering of 1 percent during the crisis), closure of special liquidity facilities for non-bank finance companies and mutual funds, increasing provisioning requirements on commercial real estate and generally, raising all provisioning cushions consisting of specific provisions against NPAs as well as floating provisions.

\section{Financial Sector}

14. The Indian banking sector turned out to be resilient in the backdrop of the global financial crisis. Apart from minimal exposure to toxic assets, the Indian banking sector has greatly benefited from the initiatives relating to adoption of counter-cyclical prudential regulations framework. Net non-performing assets (NPA) as a ratio to net advances of commercial banks as at end March 2009 remained at last year's level of 1.1 per cent. While it is not unusual to expect NPAs to increase in a downturn, Indian banks are well capitalized to cushion the impact of any possible increase in NPAs. Given the increase in banks' net worth over the past ten years and steady reduction in their NPAs, capital coverage for NPAs is at a prudent level. Furthermore, to arrest any possible increase in non-performing loans, the RBI has recently decided to increase the provisioning requirement for advances to the commercial real estate sector classified as 'standard assets' from the present level of 0.40 per cent to 1 per cent.

15. As far as long-term funding for growth is concerned, financing for infrastructure has attracted attention of the policy makers in India. The staff report has mentioned a number of new initiatives, such as, take-out financing and credit enhancements being worked on by the India Infrastructure Finance Company Limited (IIFCL). Prime Minister Dr. Manmohan Singh has aptly identified the course of reforms as,

"We need to develop long-term debt markets and to deepen corporate bond markets. This in turn calls for a strong insurance and pension sub-sectors. Some of the reforms needed, especially in insurance, involve legislative changes. We have taken initiatives in this area and will strive to build the political consensus needed for these legislative actions to be completed. We need to improve futures markets for better price discovery and regulation. We also need to remove institutional hurdles to facilitate better intermediation" (Indian Prime Minister's address at the Inauguration of India Economic Summit on November 8, 2009; available at http://pmindia.nic.in/speeches.htm). 